Synthesis of Fused Dibenzofuran Derivatives via Palladium-catalyzed Domino C-C Bond formation and Ironcatalyzed Cycloisomerization/Aromatization

Kartick Paul, Swapnadeep Jalal, Sandip Kundal and Umasish Jana*

Department of Chemistry, Jadavpur University, Kolkata 700032 ,

West Bengal, India

E-mail: jumasish2004@yahoo.co.in.

umasishjana@gmail.com,

Fax: +91-33-2414-6584

\title{
Table of content:
}

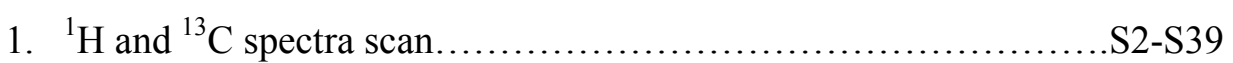

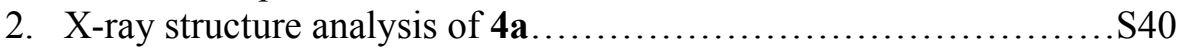


${ }^{1} \mathrm{H}$ NMR of $\mathbf{2 a}, \mathrm{CDCl}_{3}, 300 \mathrm{MHz}$

๕
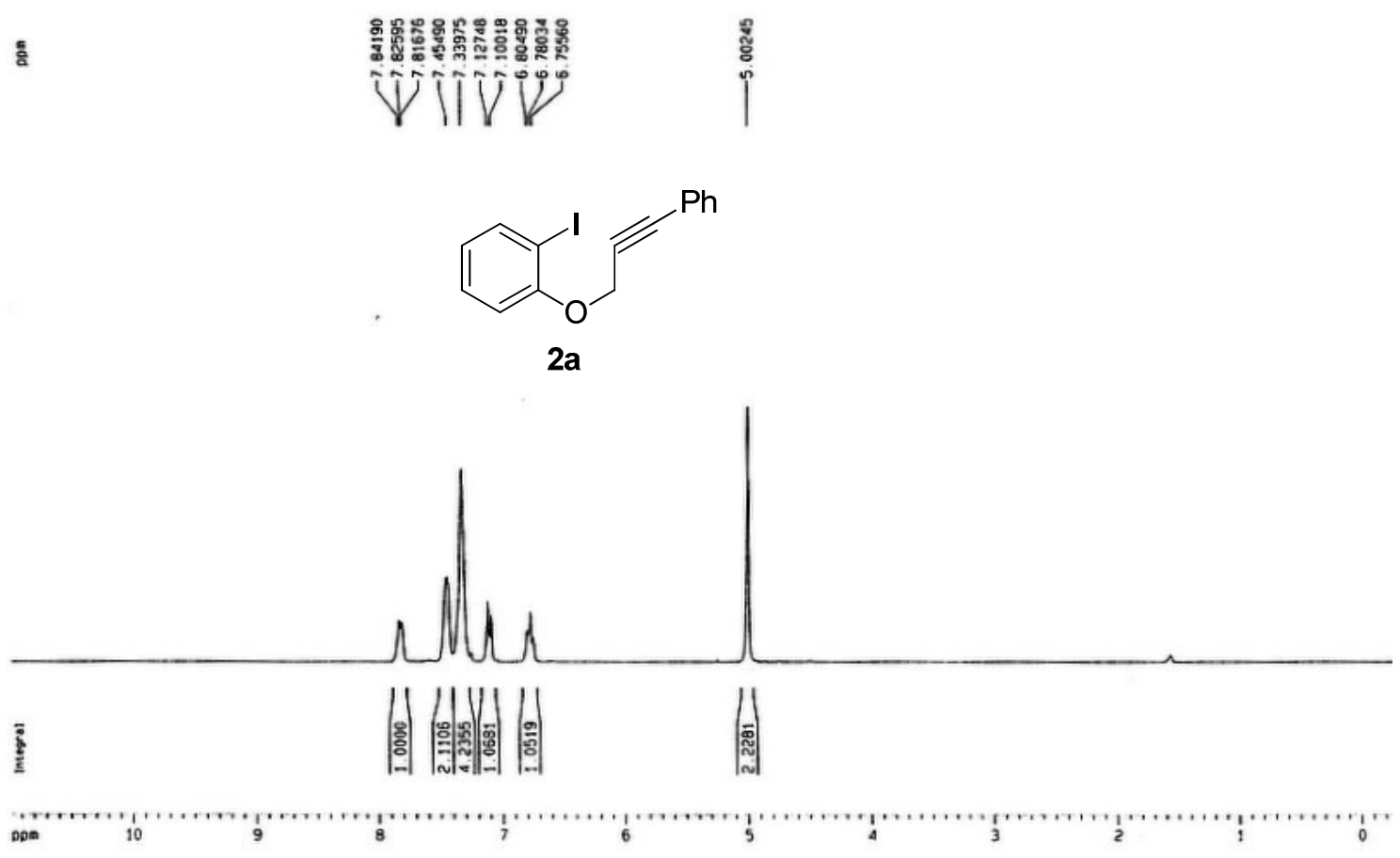

${ }^{13} \mathrm{C}$ NMR of 2a, $\mathrm{CDCl}_{3}, 75 \mathrm{MHz}$

E

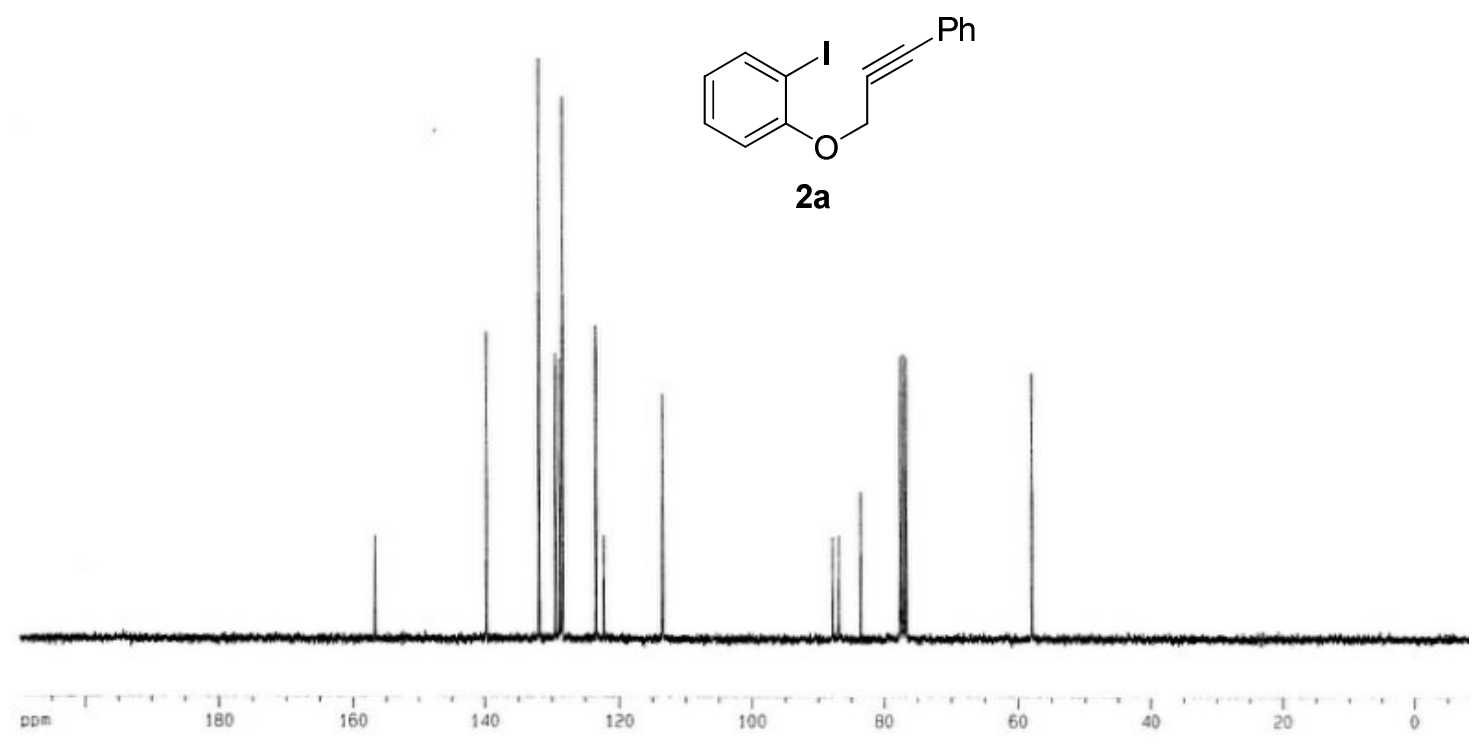


${ }^{1} \mathrm{H}$ NMR of $\mathbf{2 b}, \mathrm{CDCl}_{3}, 300 \mathrm{MHz}$

๕
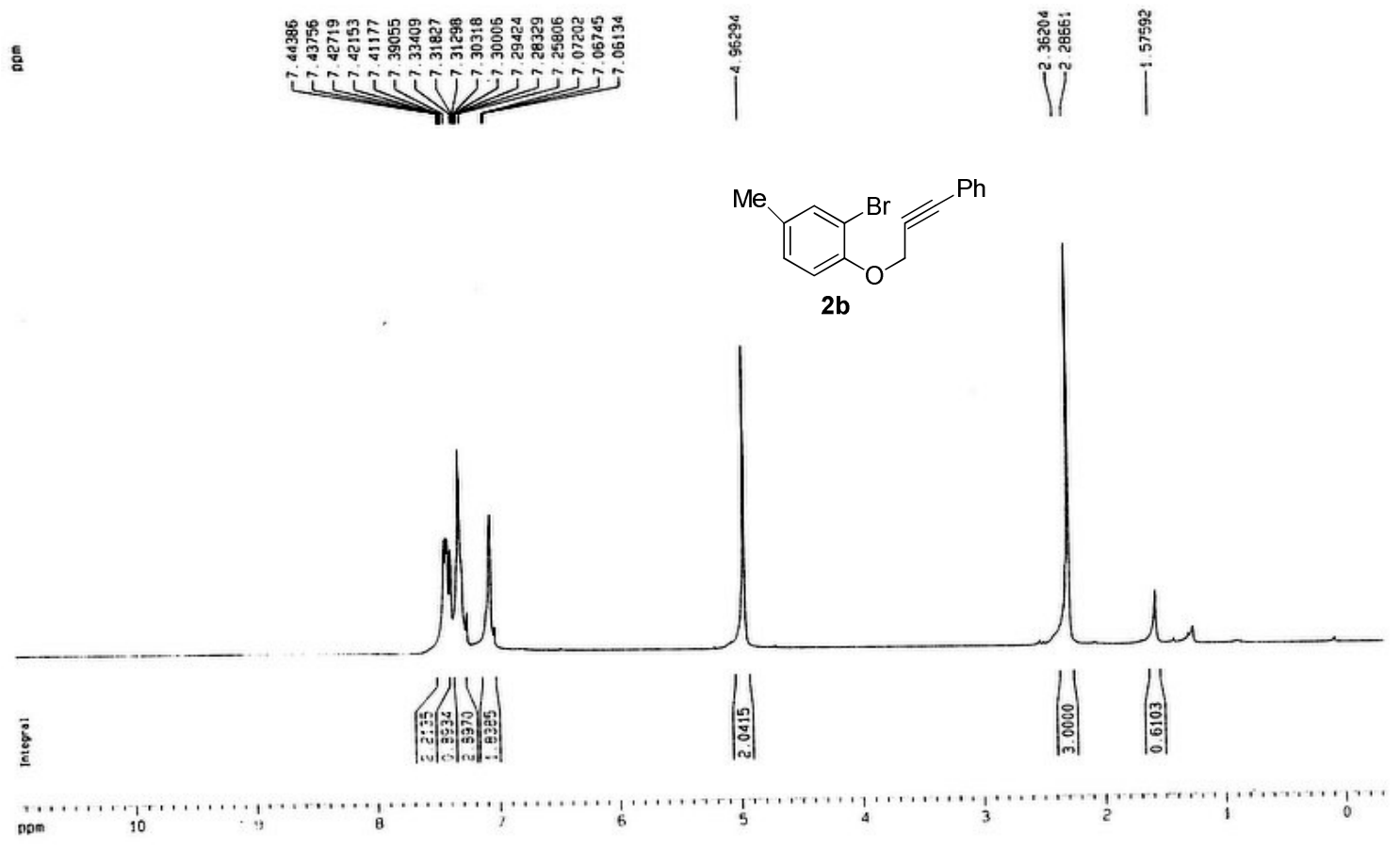

${ }^{13} \mathrm{C} \mathrm{NMR}$ of $\mathbf{2 b}, \mathrm{CDCl}_{3}, 75 \mathrm{MHz}$
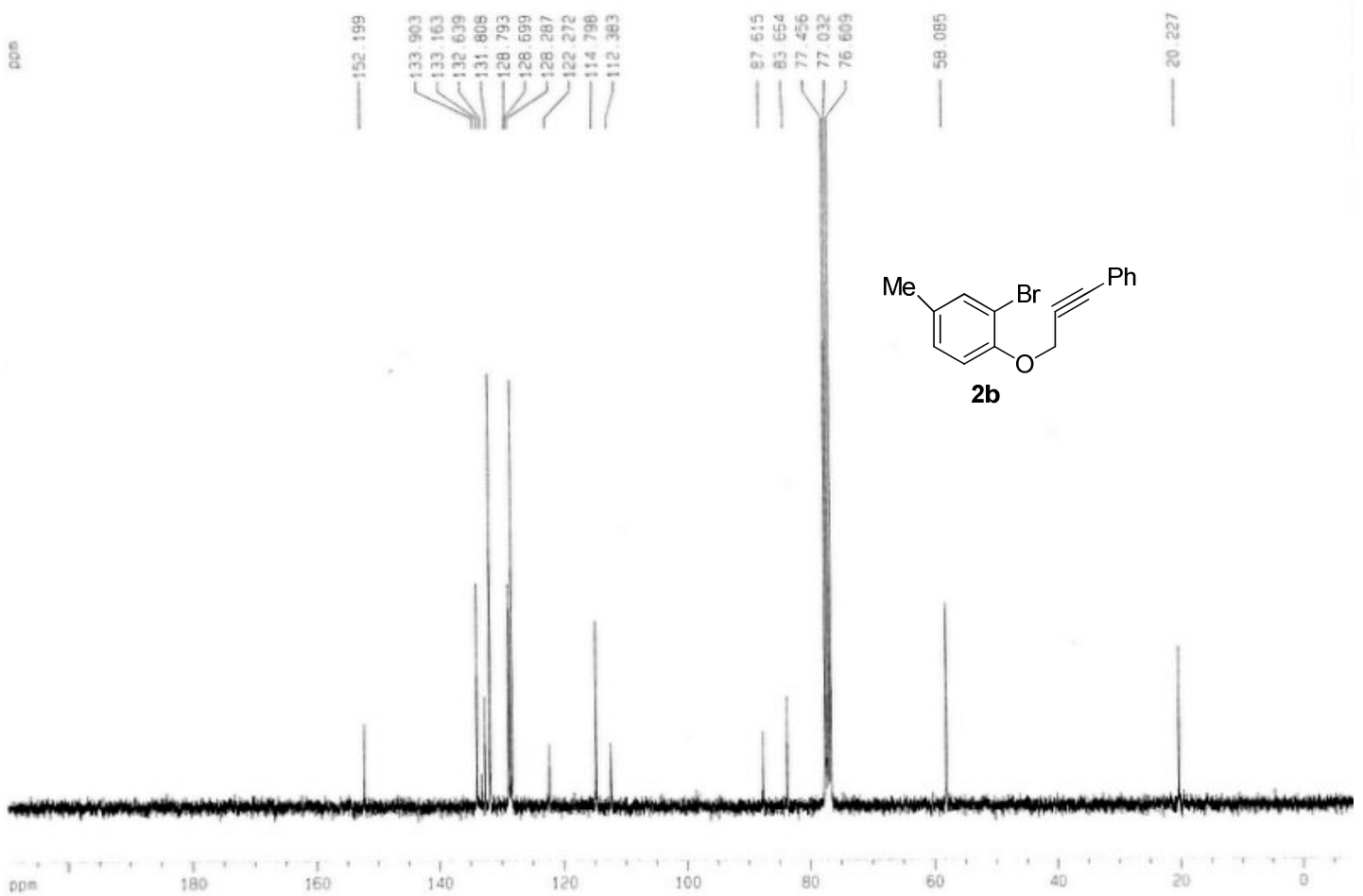
${ }^{1} \mathrm{H}$ NMR of $\mathbf{2 c}, \mathrm{CDCl}_{3} 300 \mathrm{MHz}$

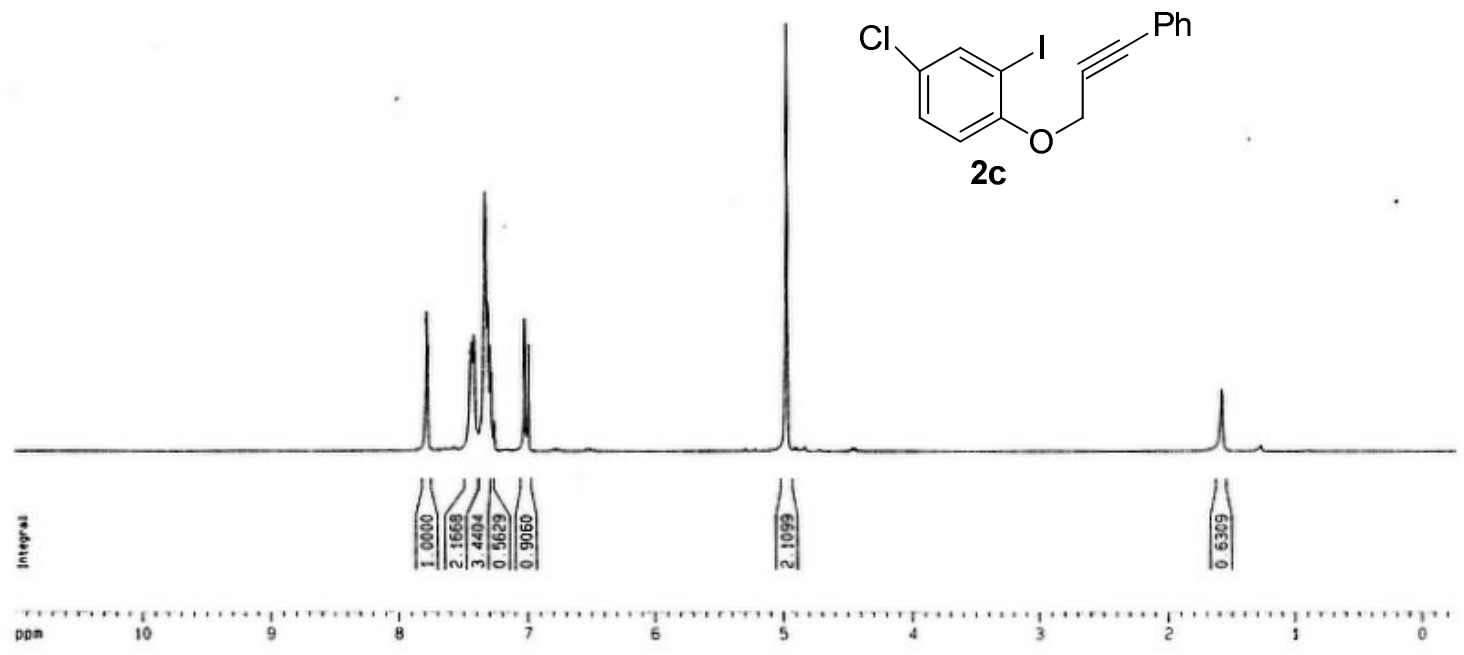

a

${ }^{13} \mathrm{C}$ NMR of $\mathbf{2 c}, \mathrm{CDCl}_{3}, 75 \mathrm{MHz}$

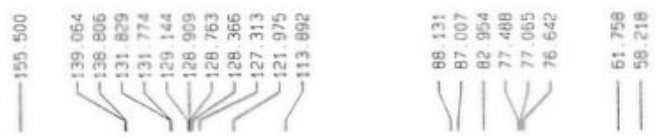

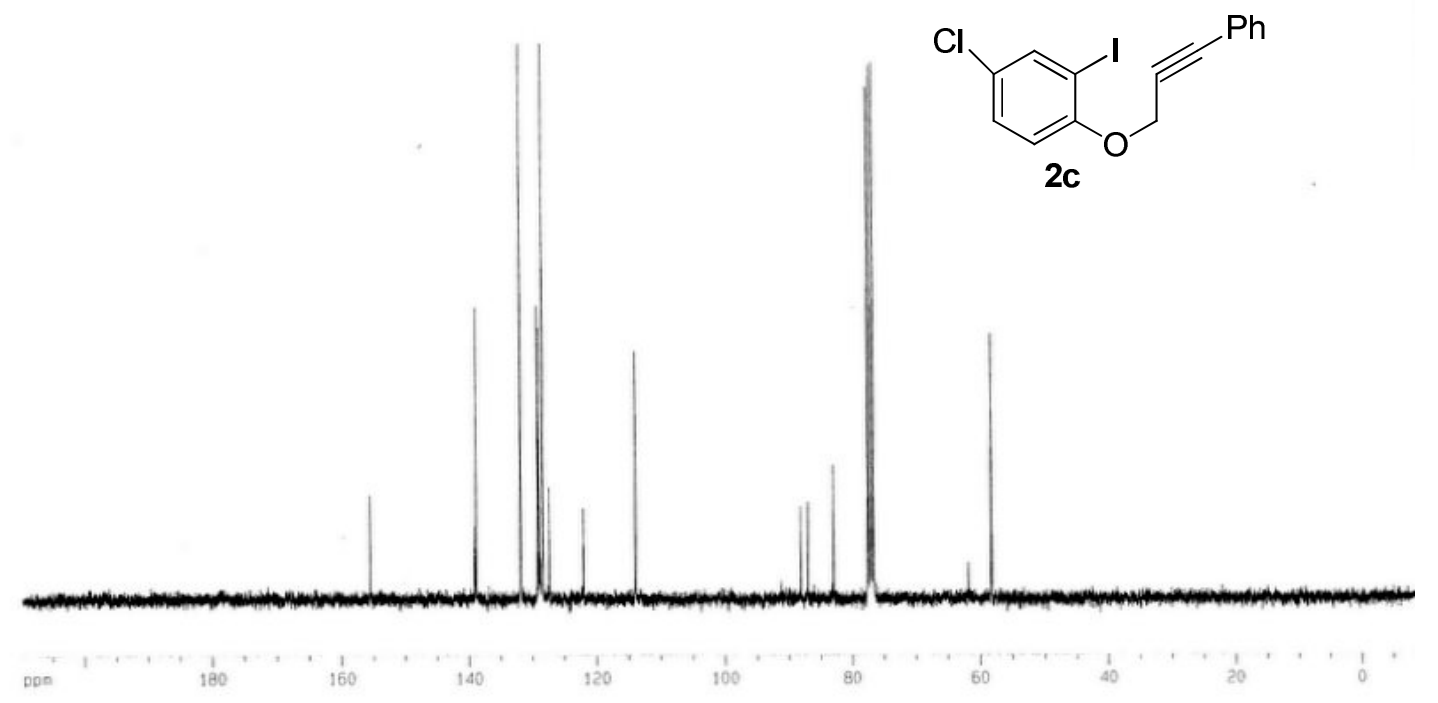


${ }^{1} \mathrm{H}$ NMR of 2d, $\mathrm{CDCl}_{3}, 300 \mathrm{MHz}$

$\mathrm{s}$
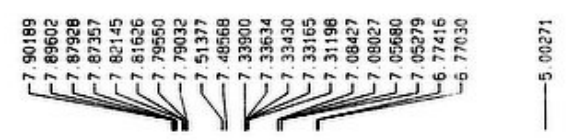

ๆ
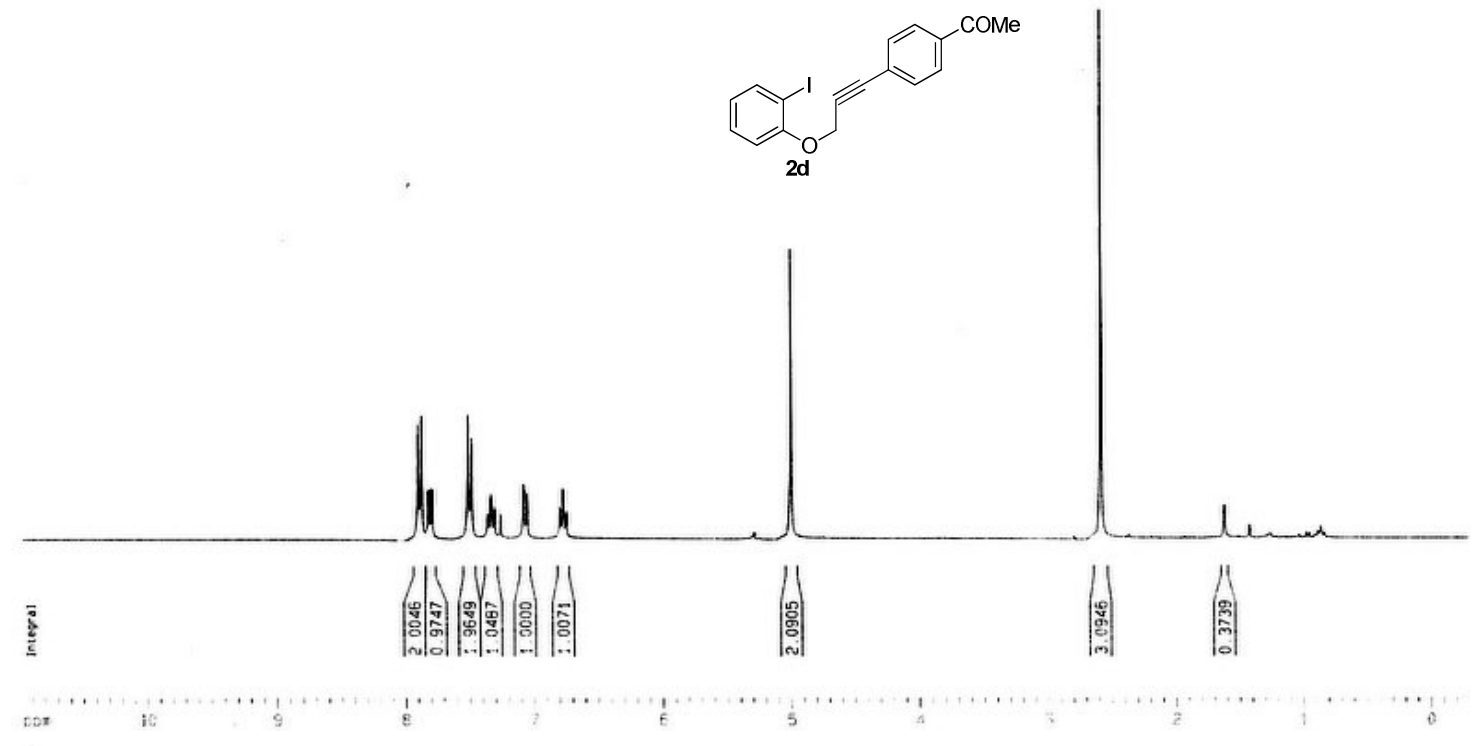

${ }^{13} \mathrm{C} \mathrm{NMR}$ of 2d, $\mathrm{CDCl}_{3}, 75 \mathrm{MHz}$
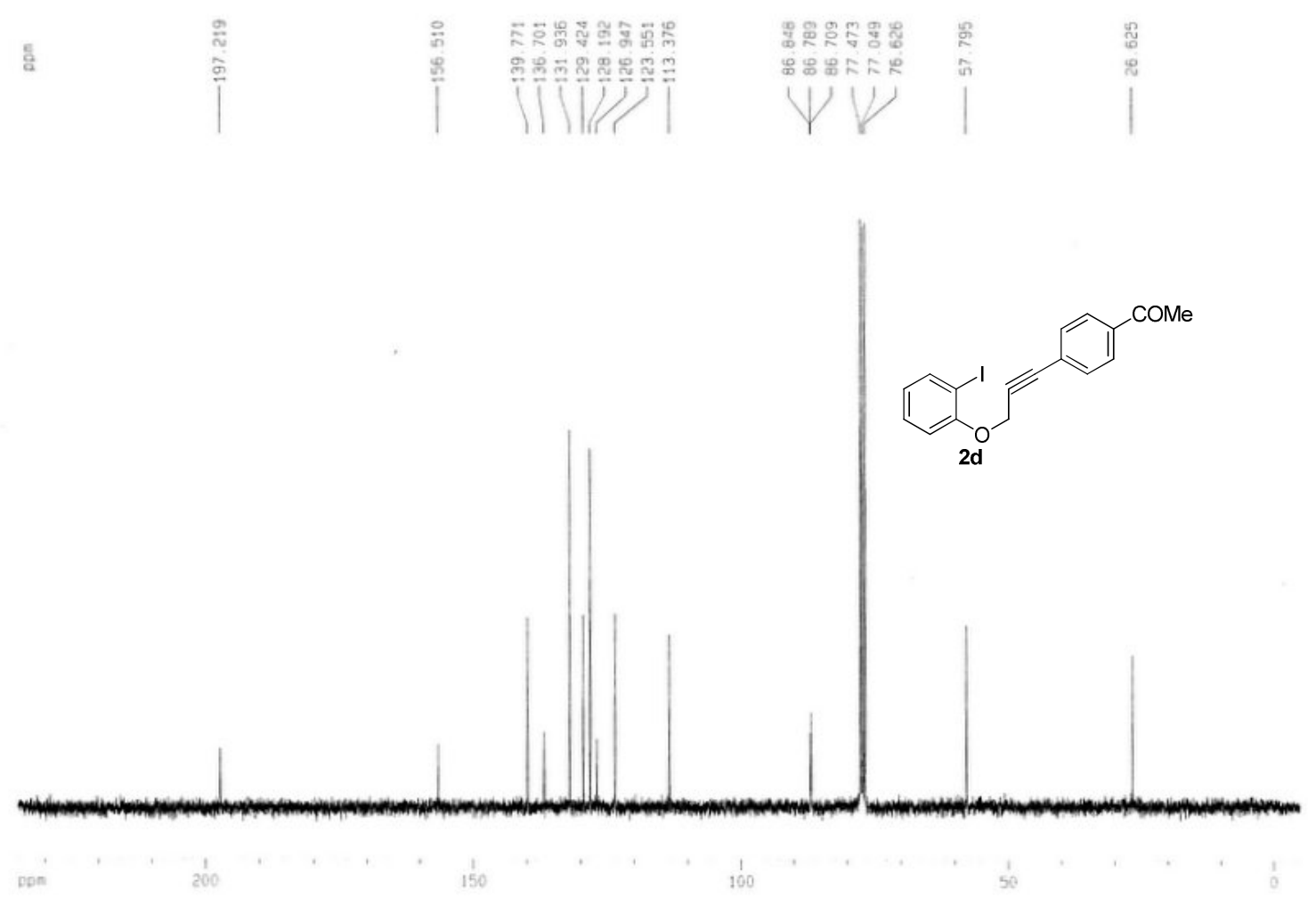
${ }^{1} \mathrm{H} \mathrm{NMR}$ of $\mathbf{2 e}, \mathrm{CDCl}_{3}, 300 \mathrm{MHz}$
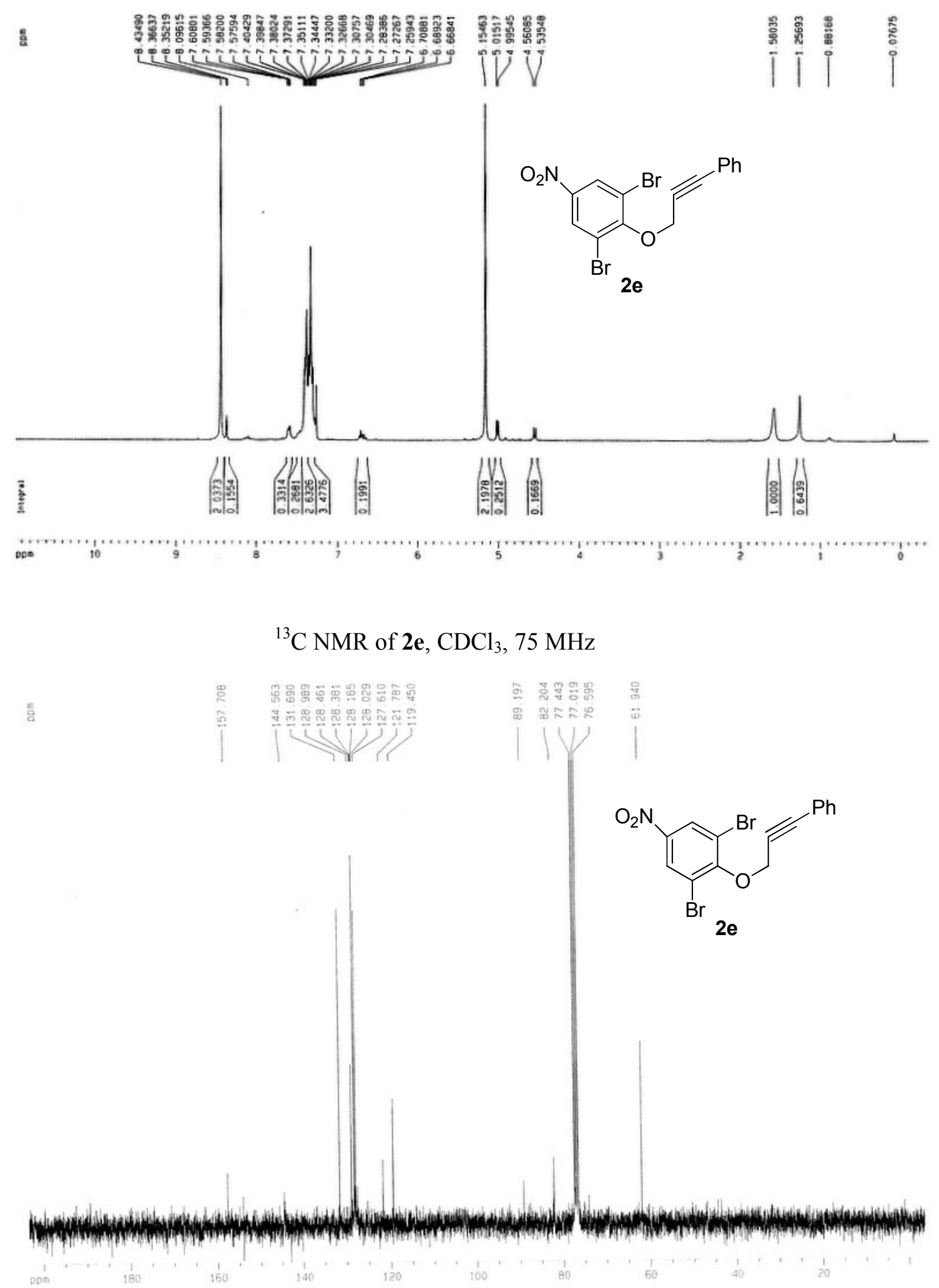
${ }^{1} \mathrm{H}$ NMR of $2 \mathbf{f}, \mathrm{CDCl}_{3}, 300 \mathrm{MHz}$

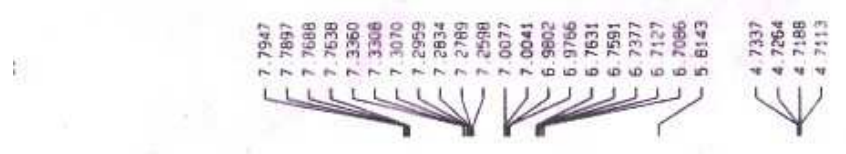

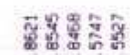

(j)

YV

ธิे

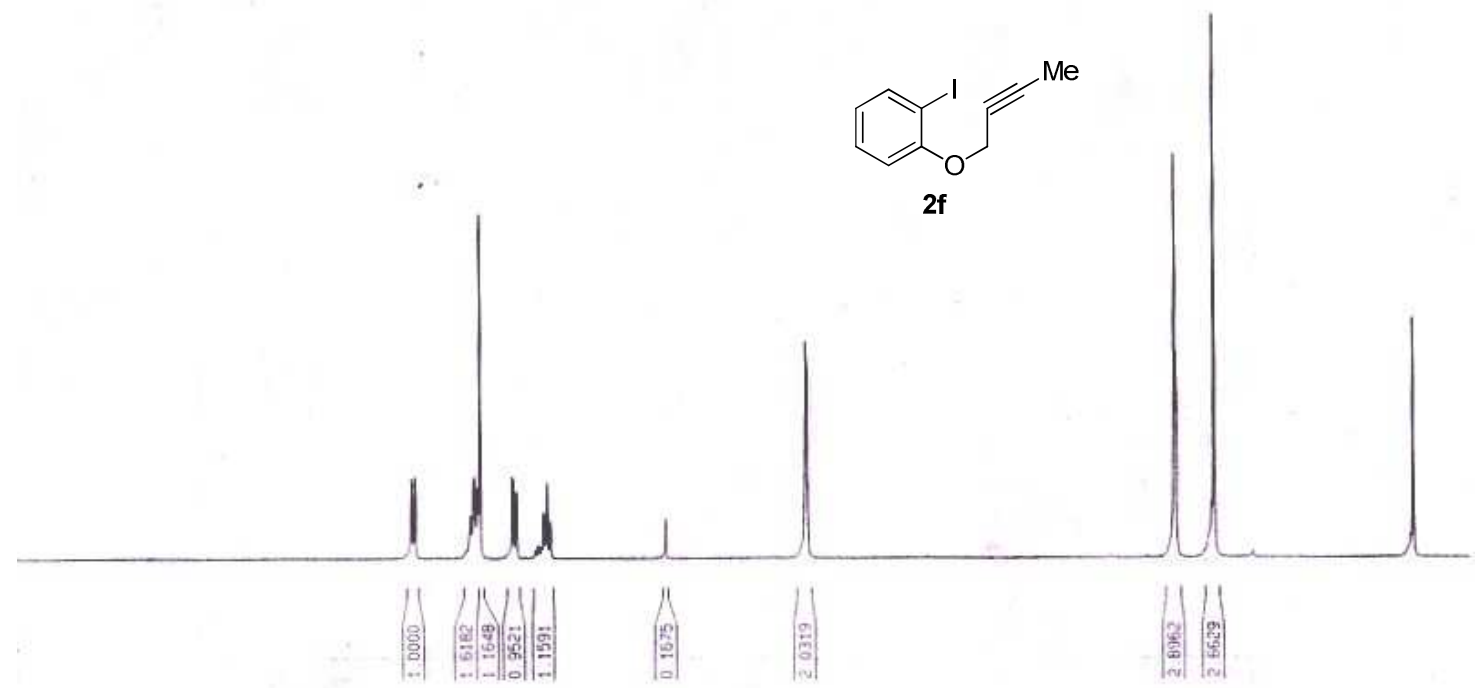

${ }^{13} \mathrm{C} \mathrm{NMR}$ of $\mathbf{2 f}, \mathrm{CDCl}_{3}, 75 \mathrm{MHz}$
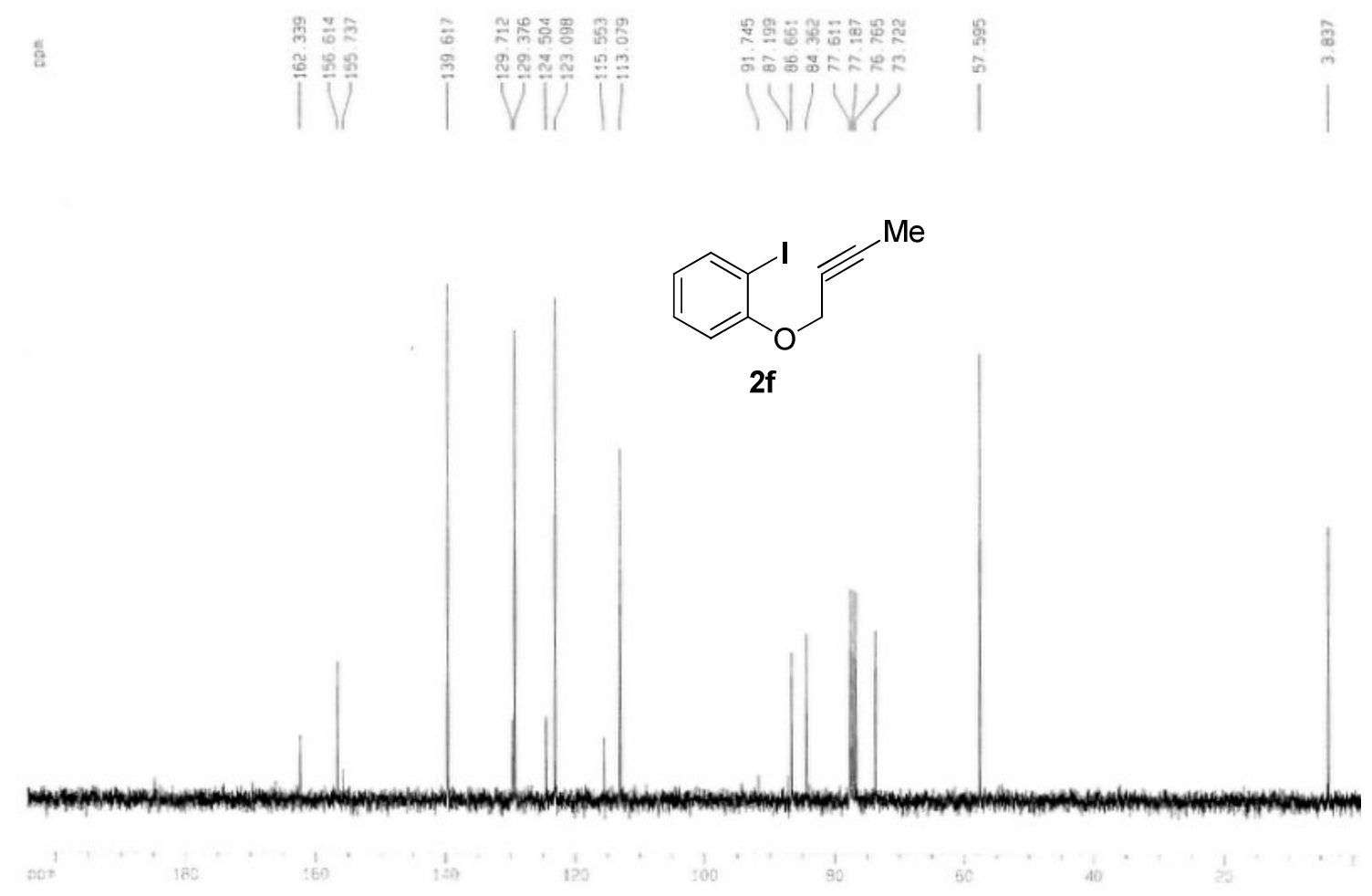


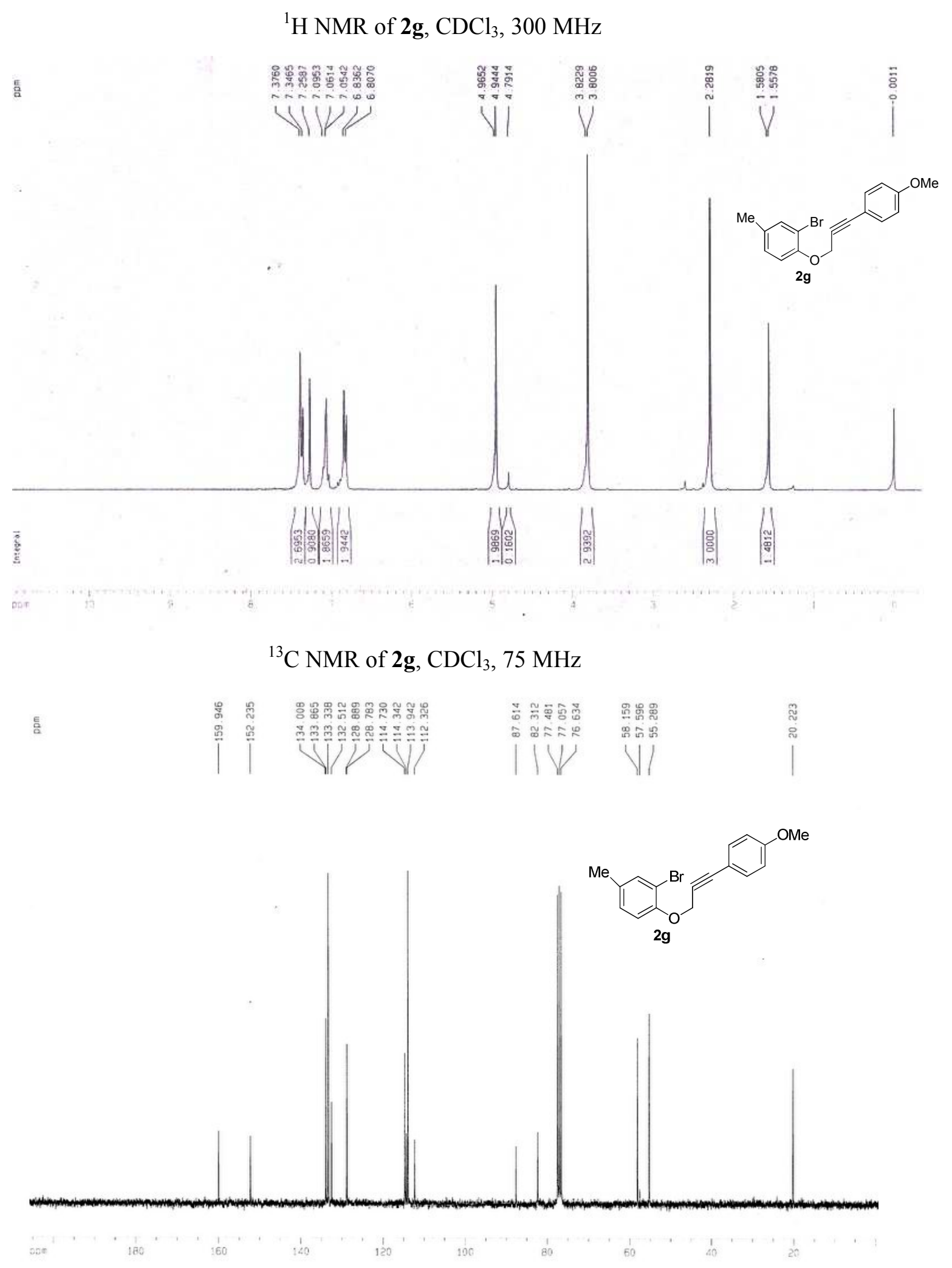




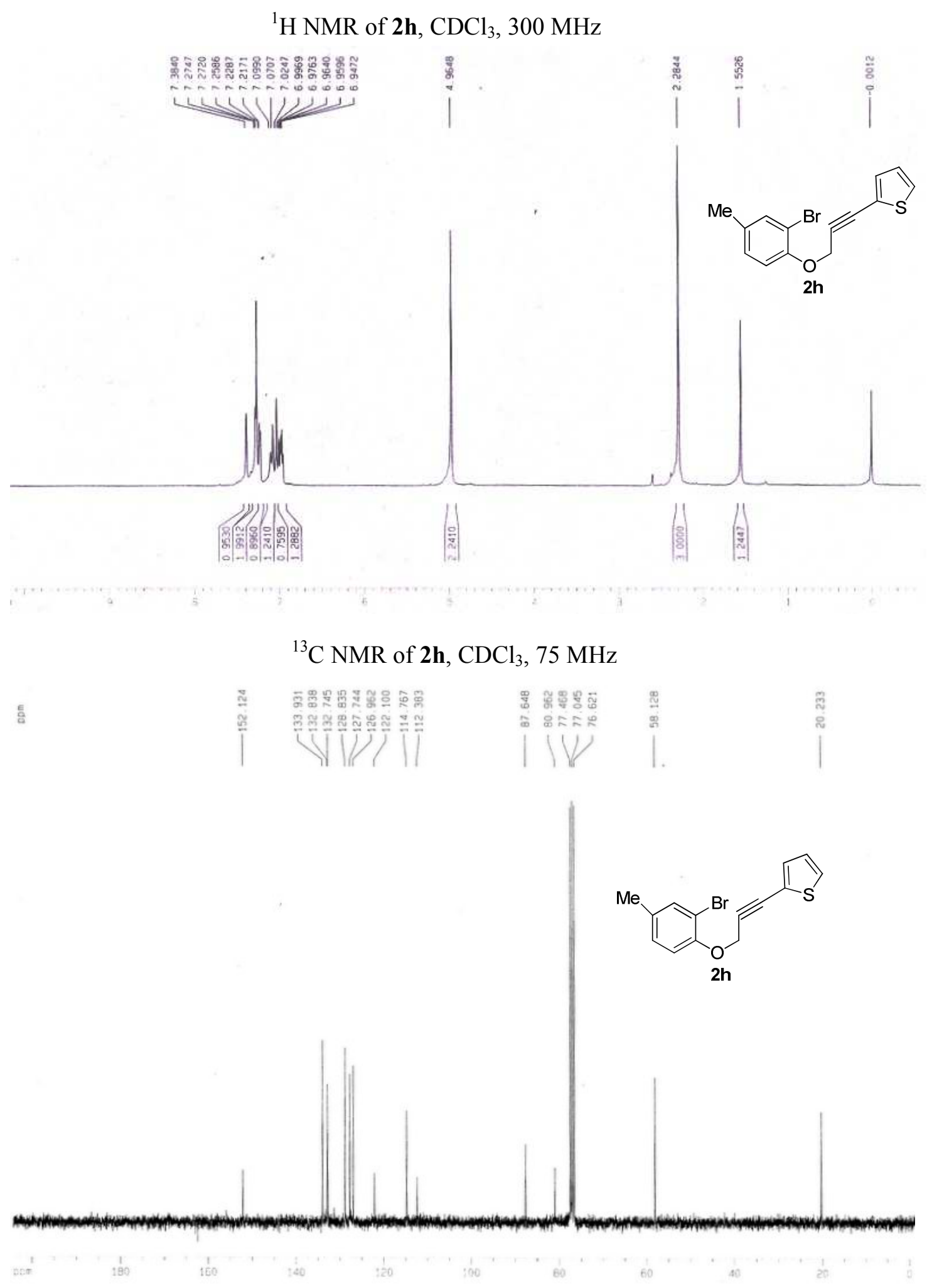



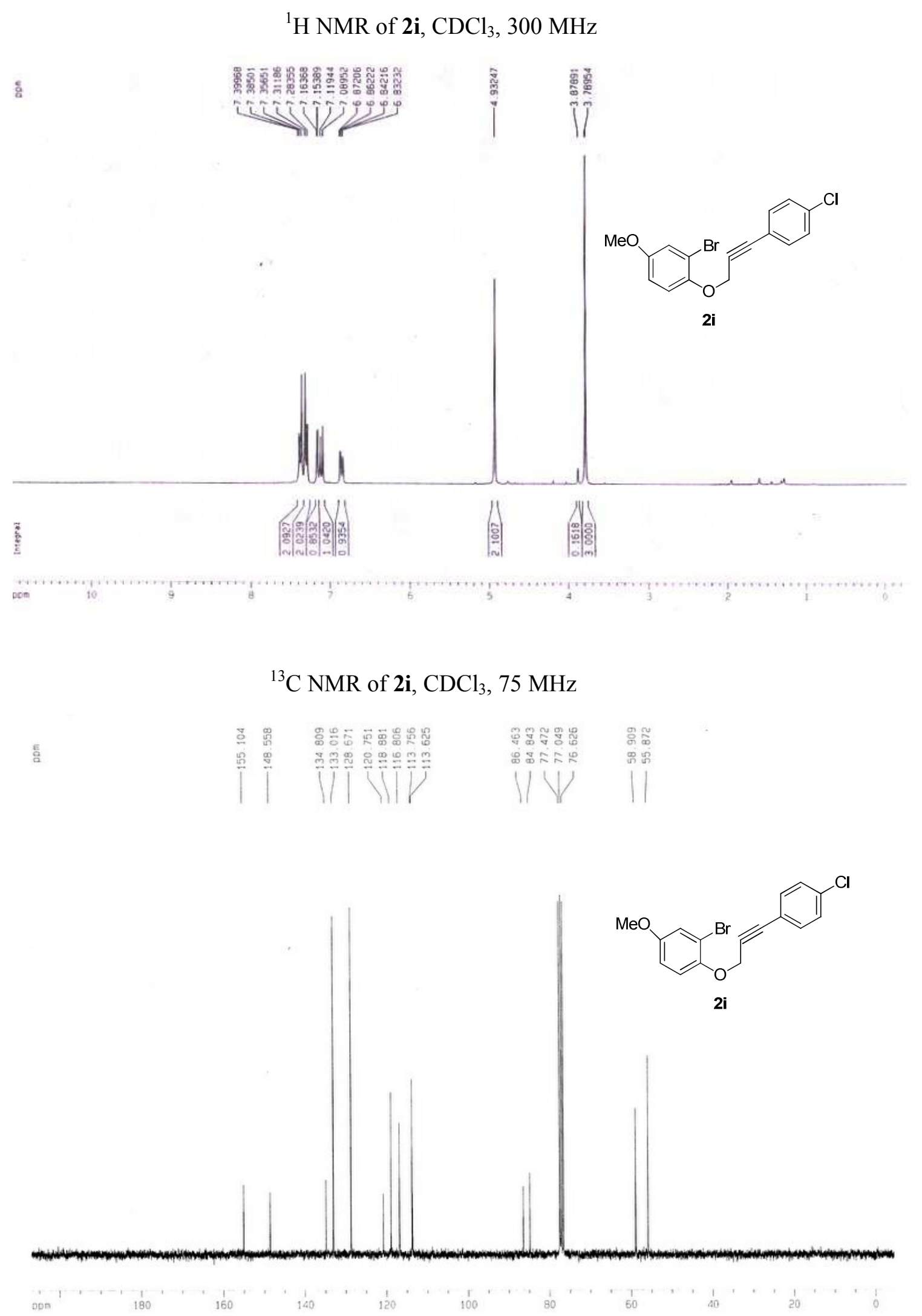


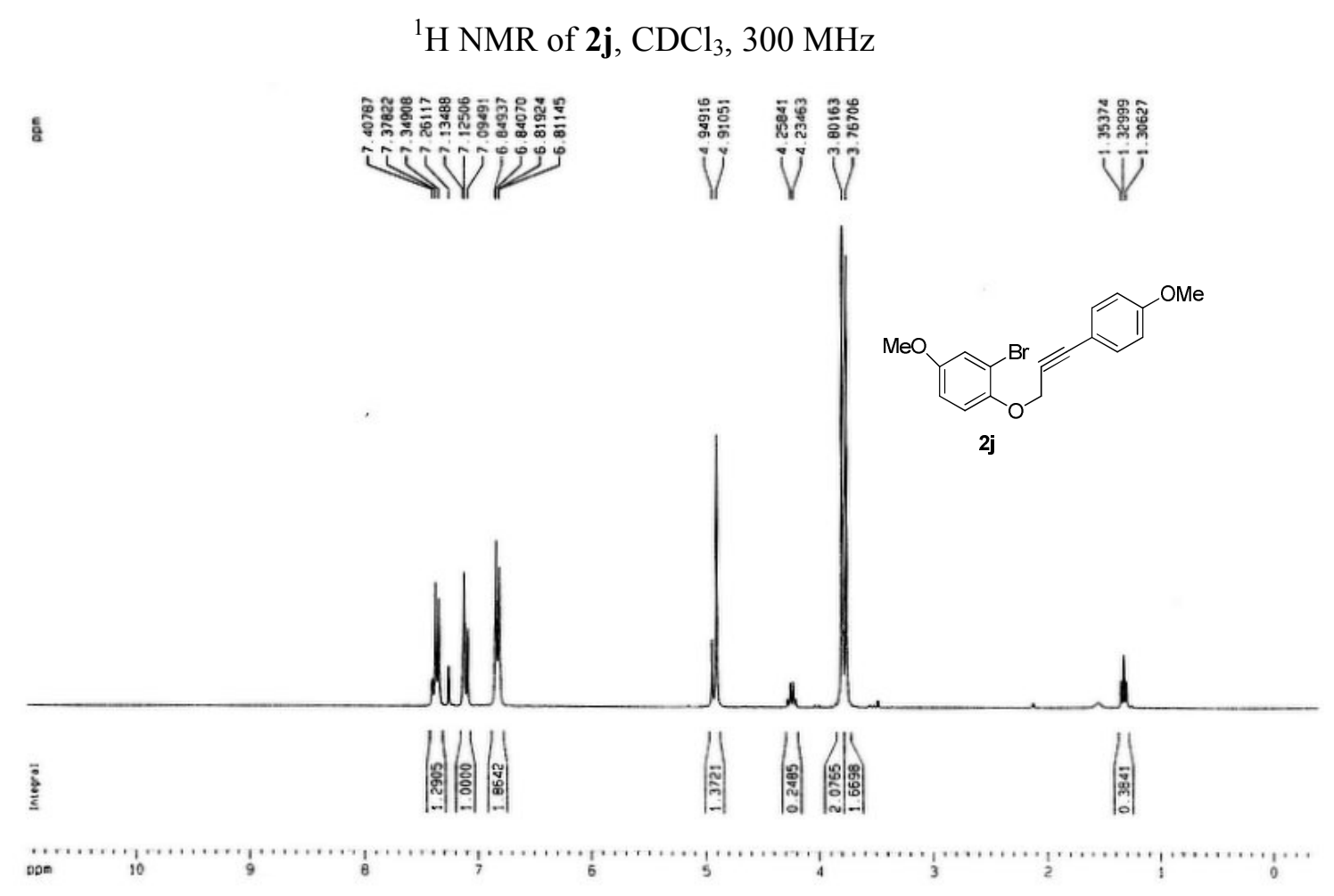

${ }^{13} \mathrm{C} \mathrm{NMR}$ of $\mathbf{2 j}, \mathrm{CDCl}_{3}, 75 \mathrm{MHz}$

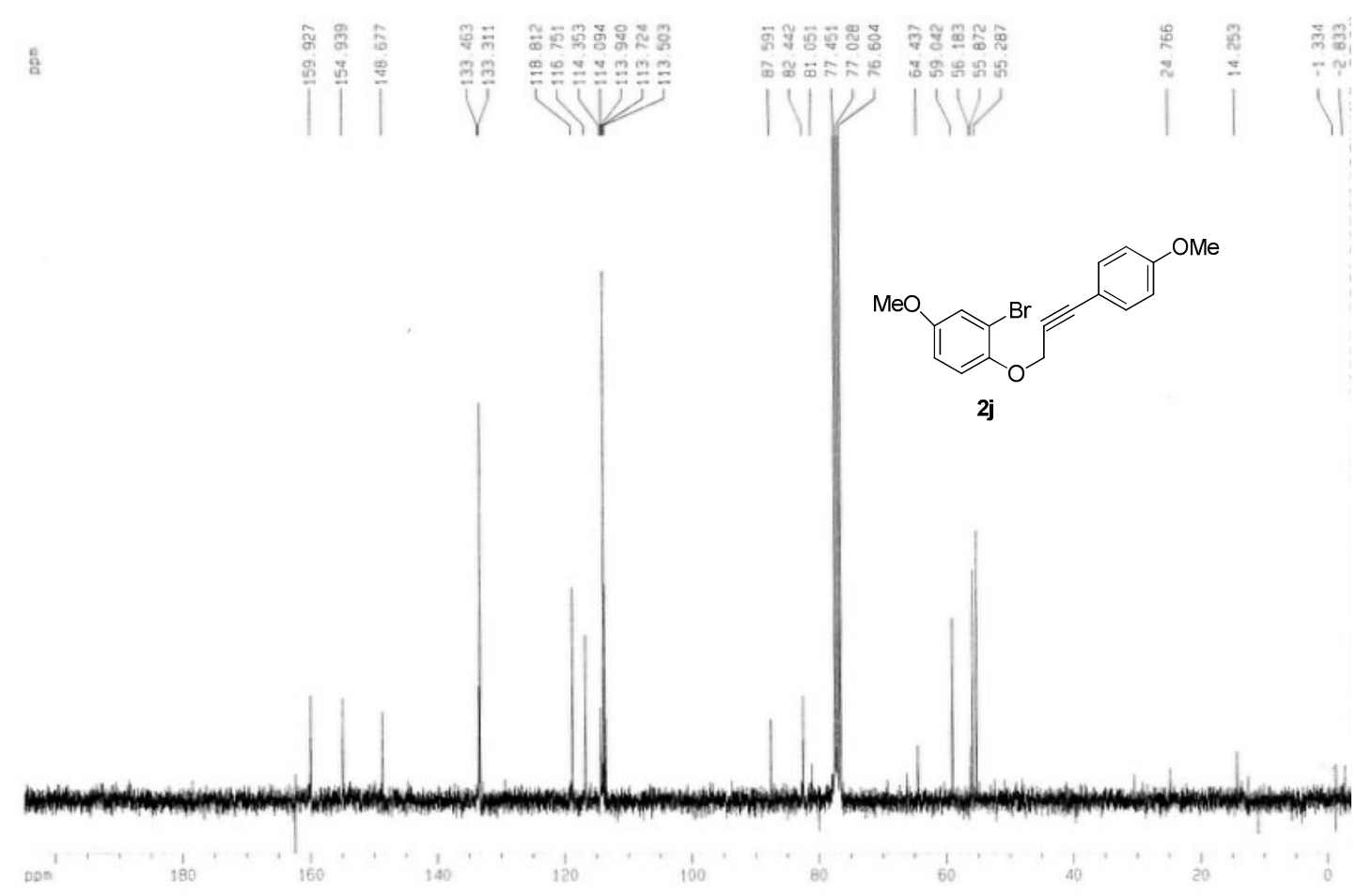


${ }^{1} \mathrm{H}$ NMR of $\mathbf{2 k}, \mathrm{CDCl}_{3}, 300 \mathrm{MHz}$

๕̊
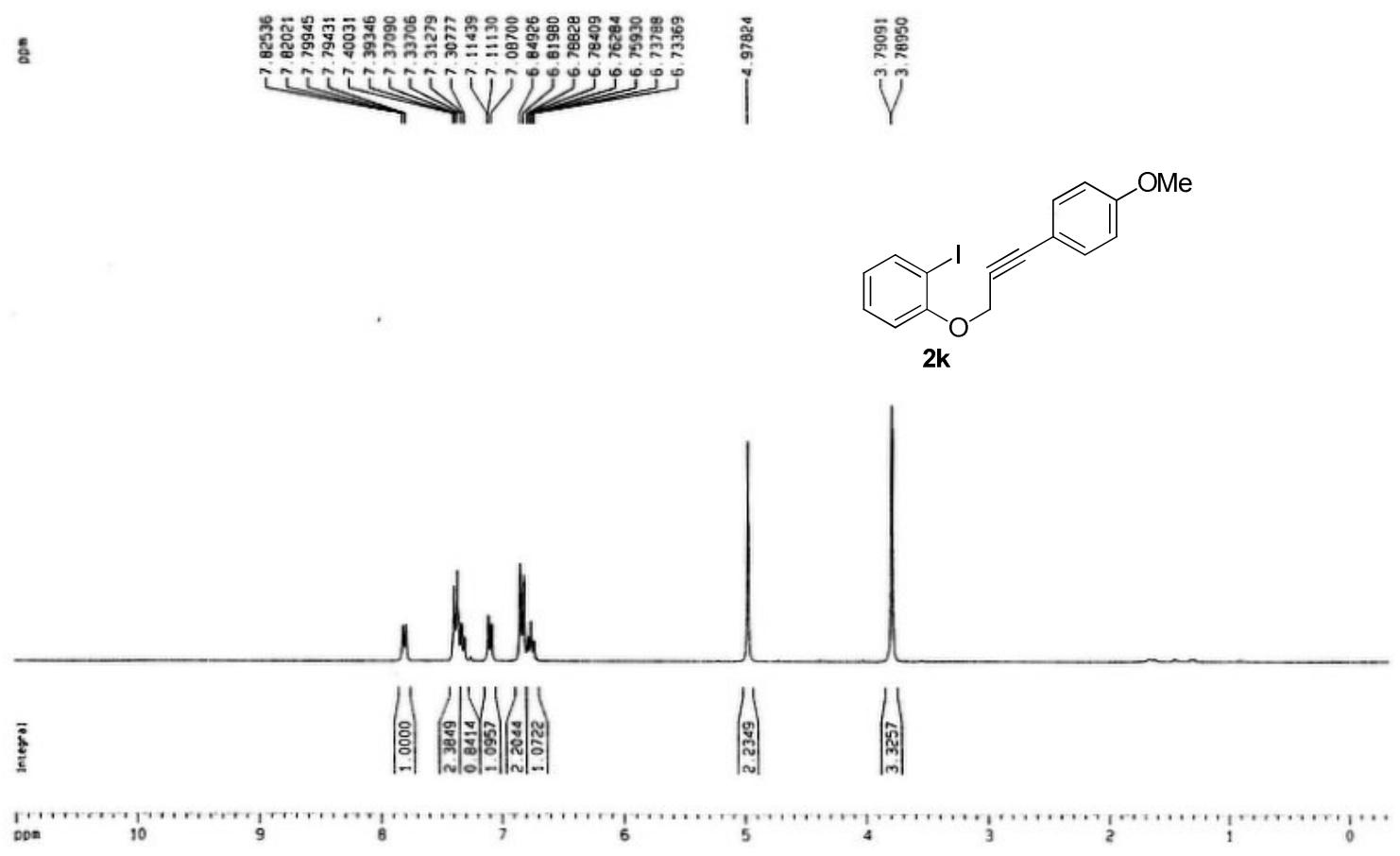

${ }^{13} \mathrm{C} \mathrm{NMR}$ of $\mathbf{2 k}, \mathrm{CDCl}_{3}, 75 \mathrm{MHz}$

a

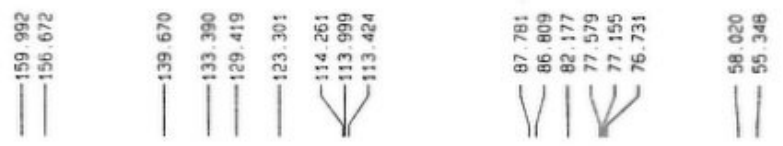

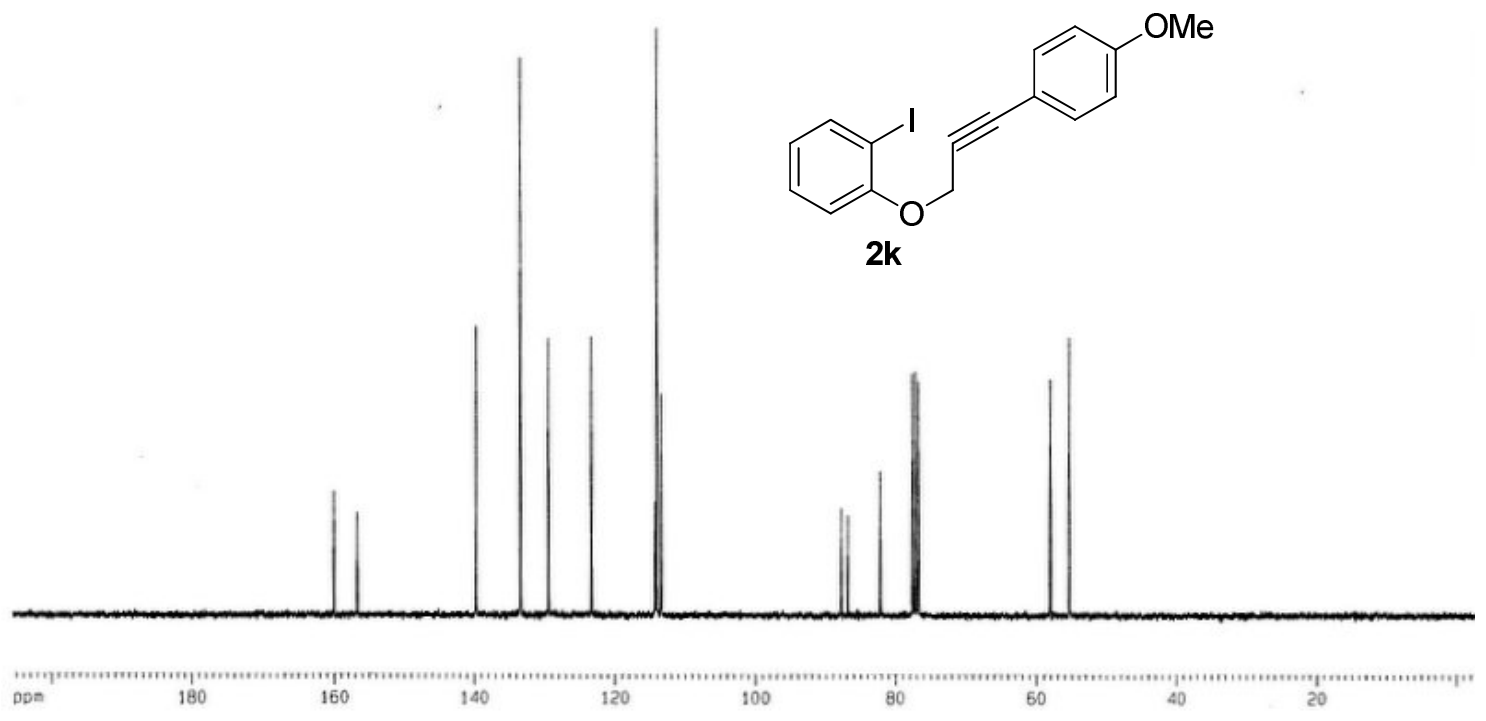




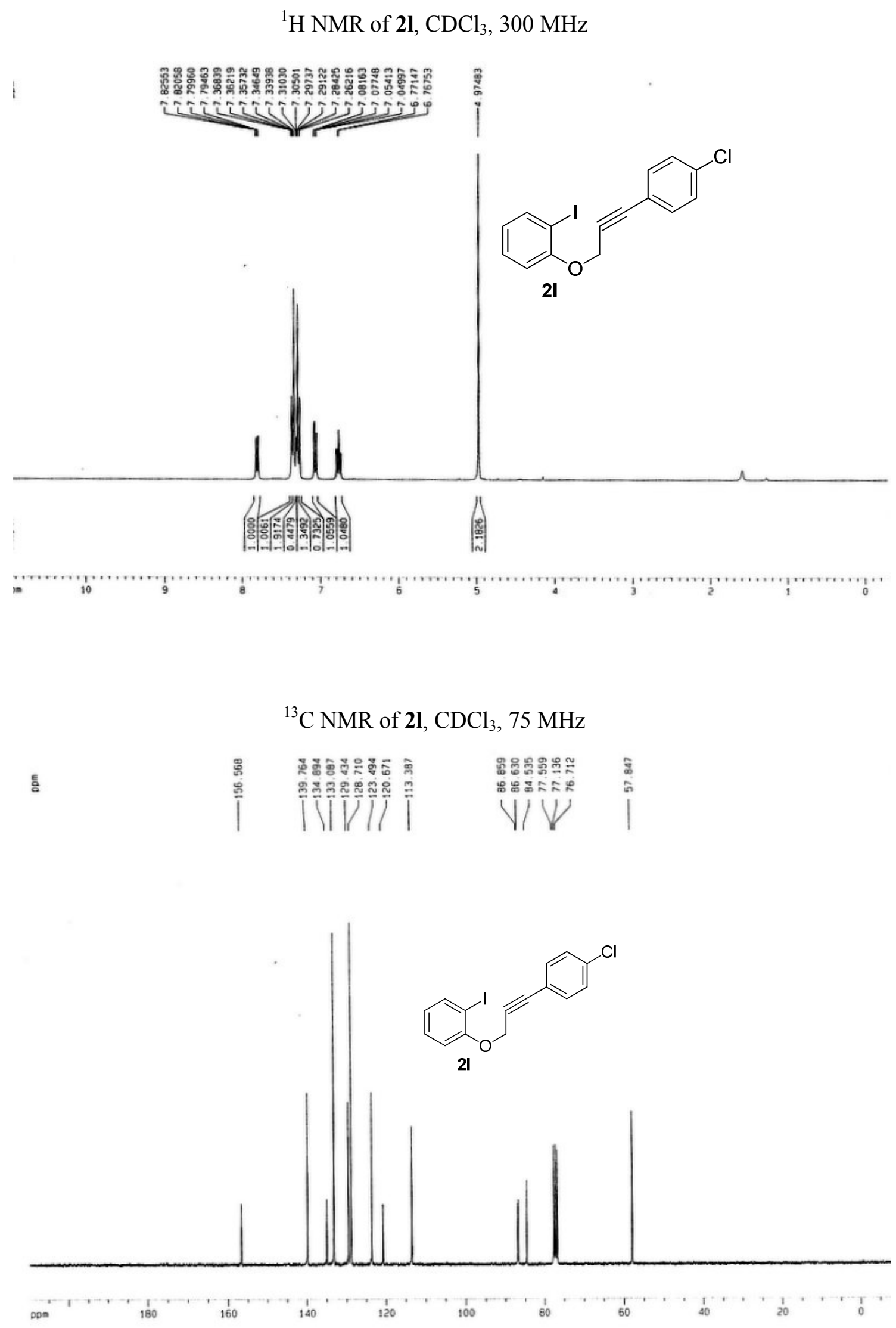




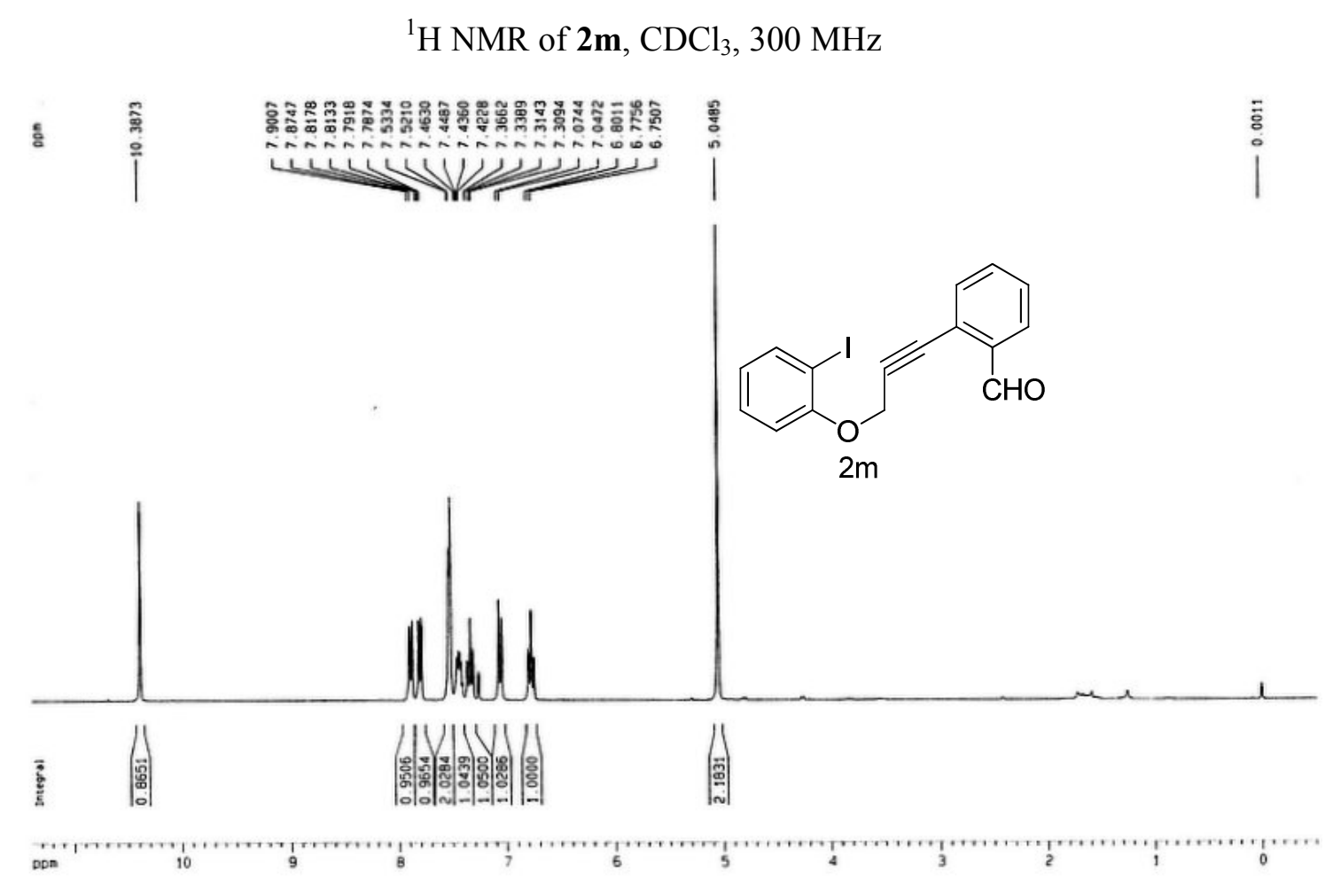

${ }^{13} \mathrm{C} \mathrm{NMR}$ of $\mathbf{2 m}, \mathrm{CDCl}_{3}, 75 \mathrm{MHz}$
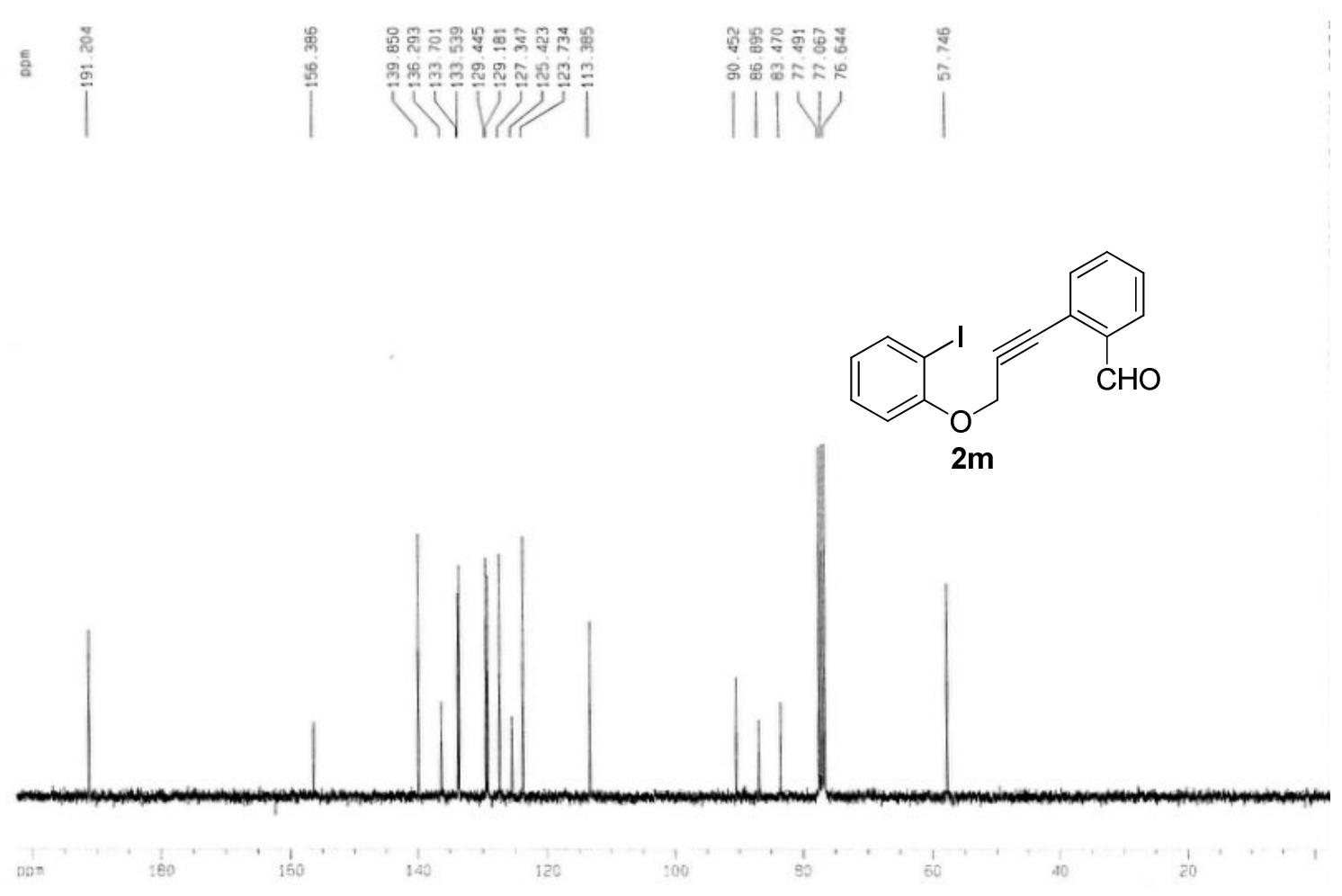


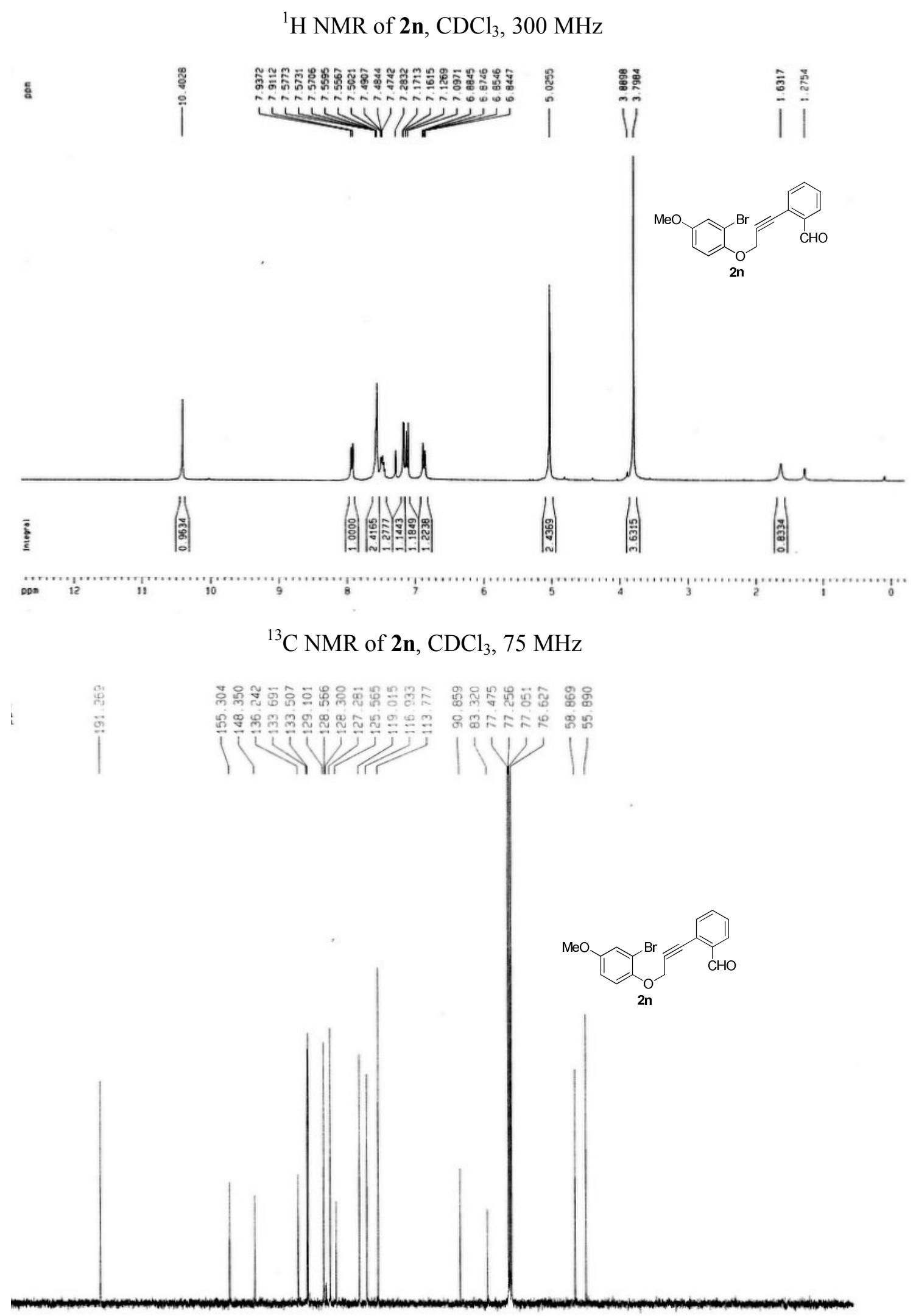



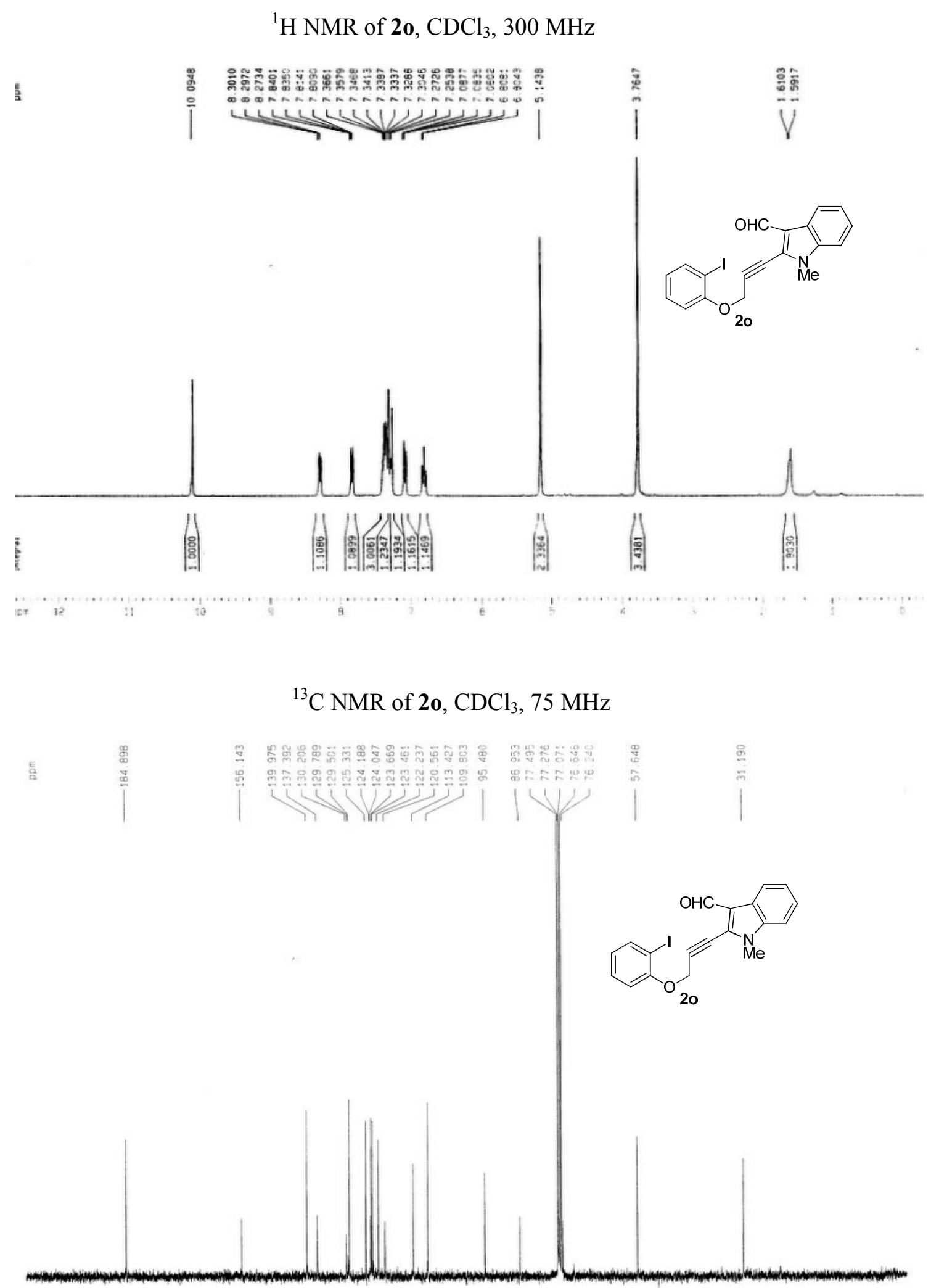

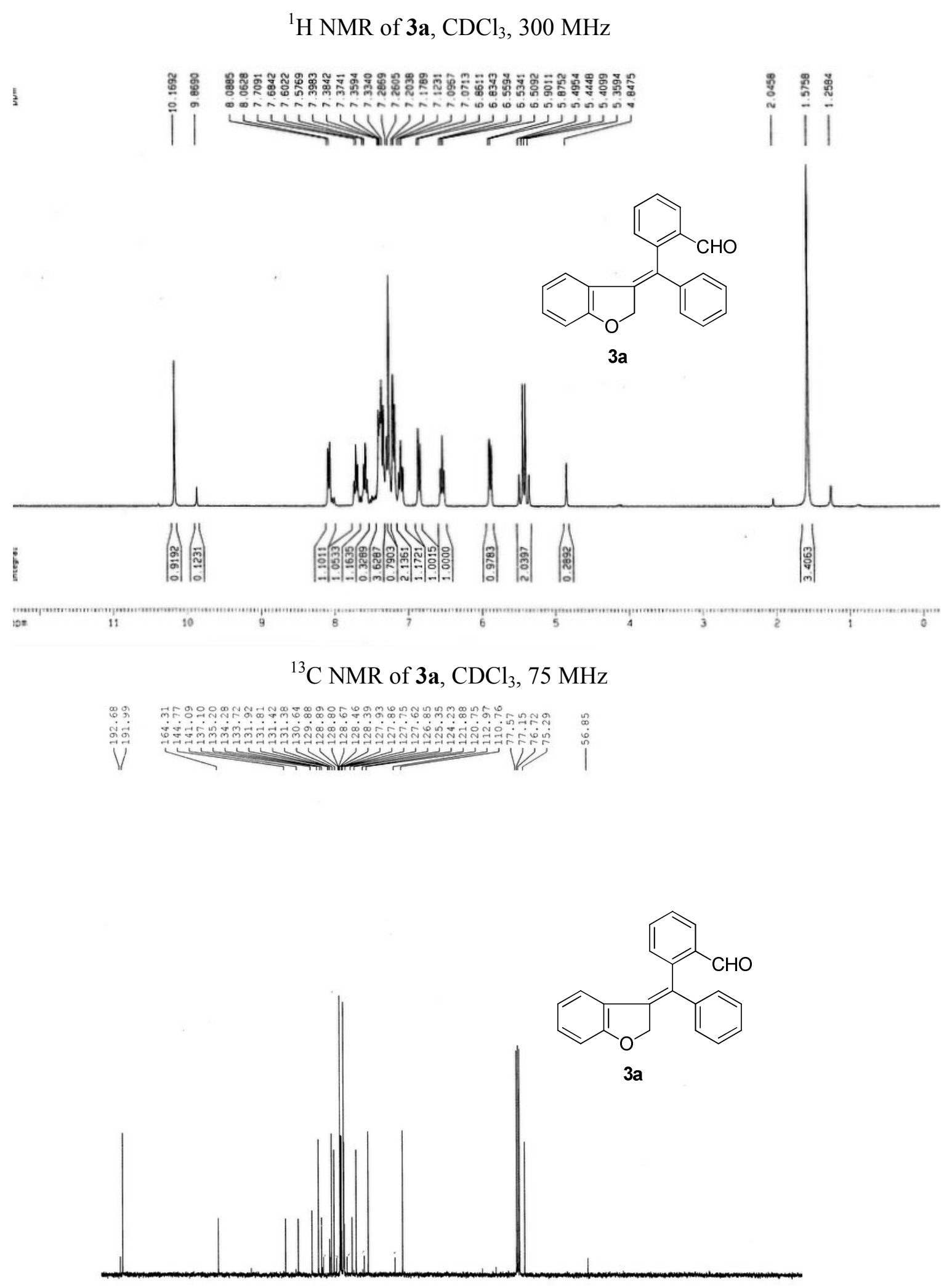
${ }^{1} \mathrm{H} \mathrm{NMR}$ of $\mathbf{4 a}, \mathrm{CDCl}_{3}, 300 \mathrm{MHz}$

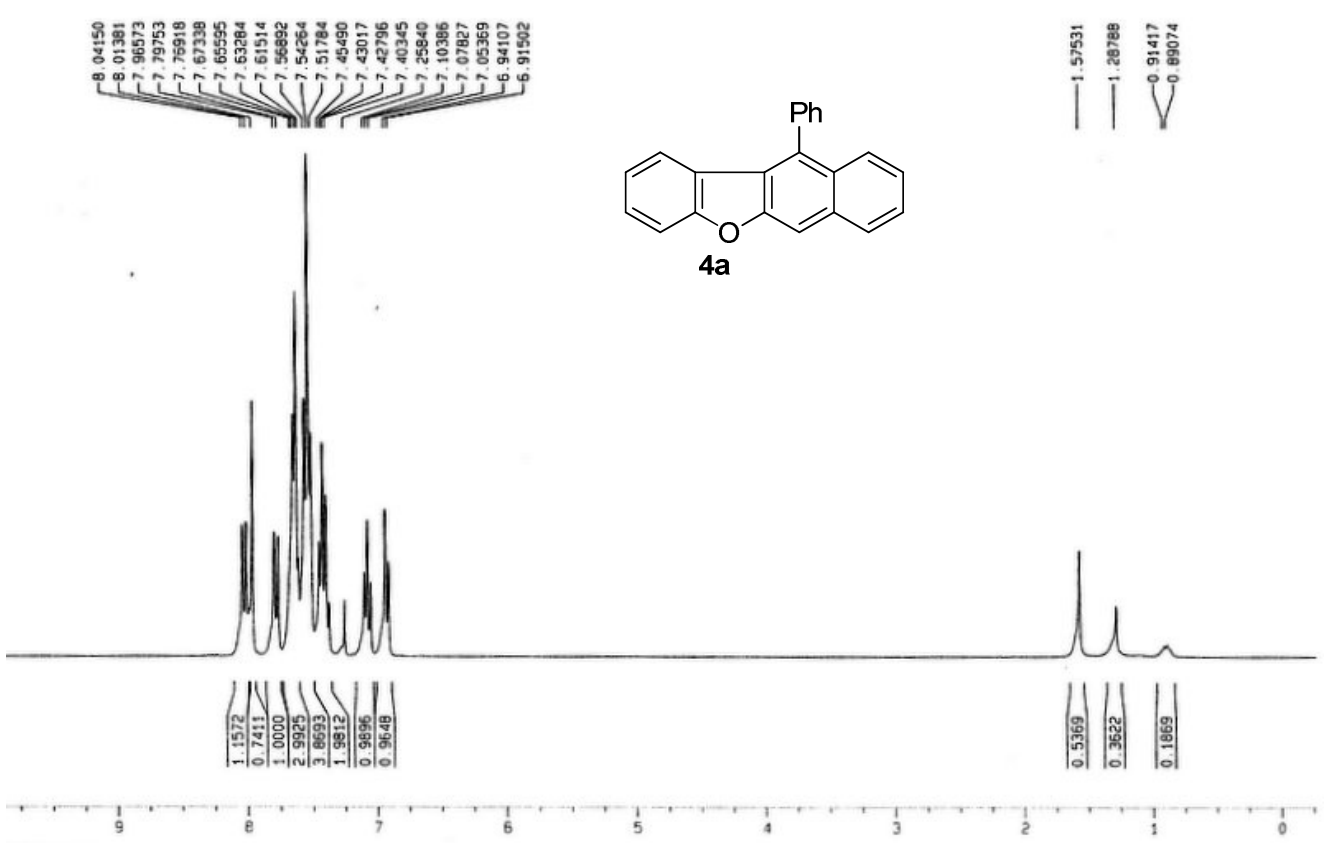

${ }^{13} \mathrm{C} \mathrm{NMR}$ of $4 \mathbf{a}, \mathrm{CDCl}_{3}, 75 \mathrm{MHz}$
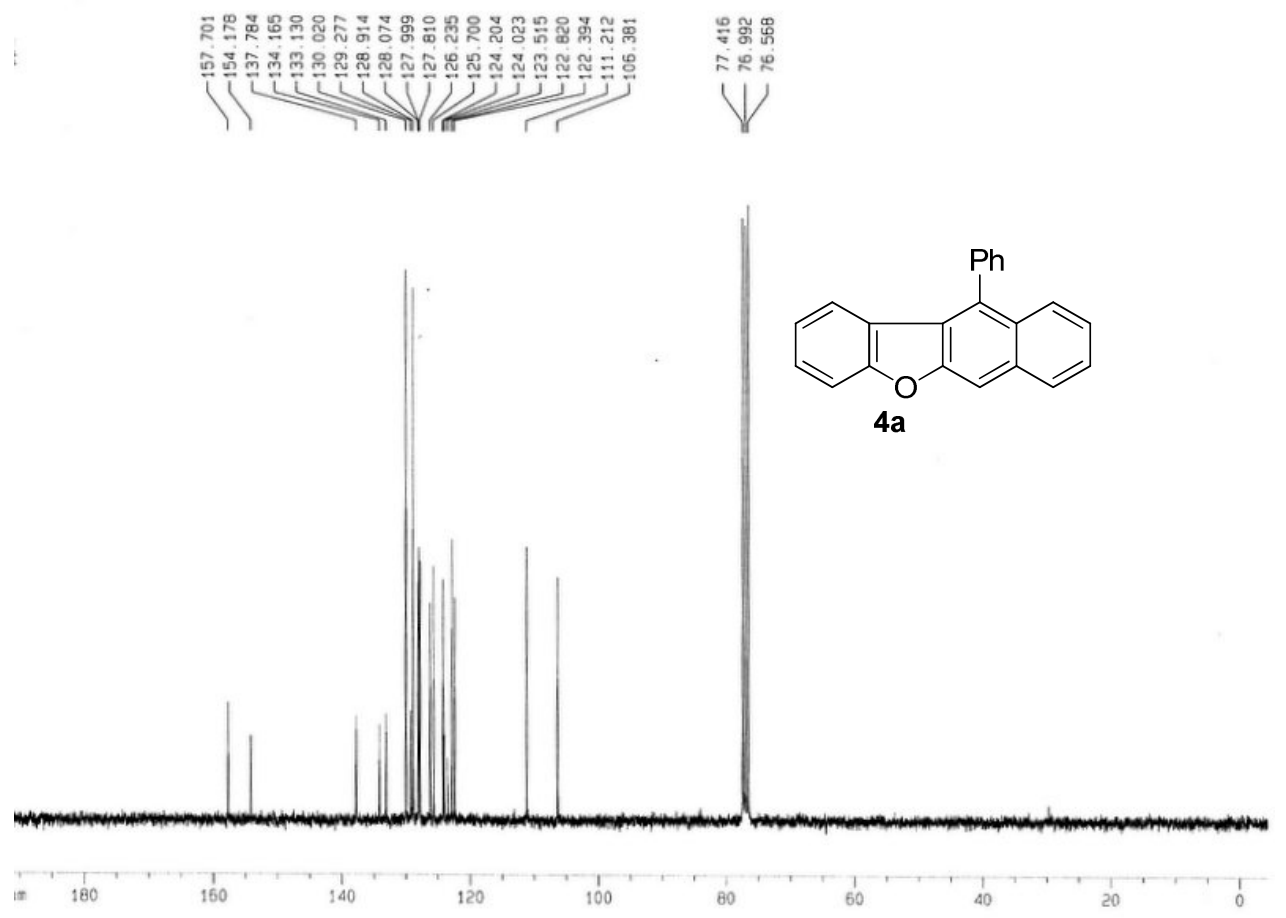
${ }^{1} \mathrm{H}$ NMR of $\mathbf{4 b}, \mathrm{CDCl}_{3}, 300 \mathrm{MHz}$

ธิ

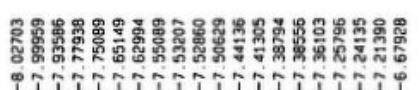

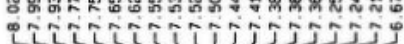

तो<smiles></smiles>

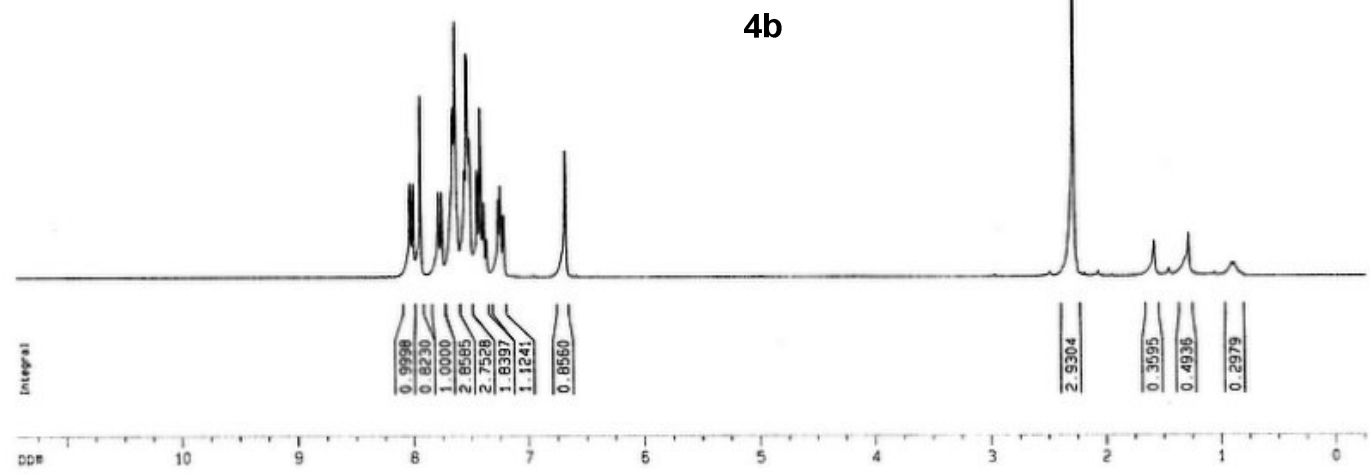

${ }^{13} \mathrm{C}$ NMR of $\mathbf{4 b}, \mathrm{CDCl}_{3}, 100 \mathrm{MHz}$

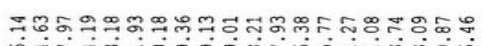

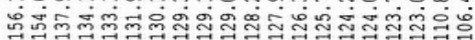

11

棺

|

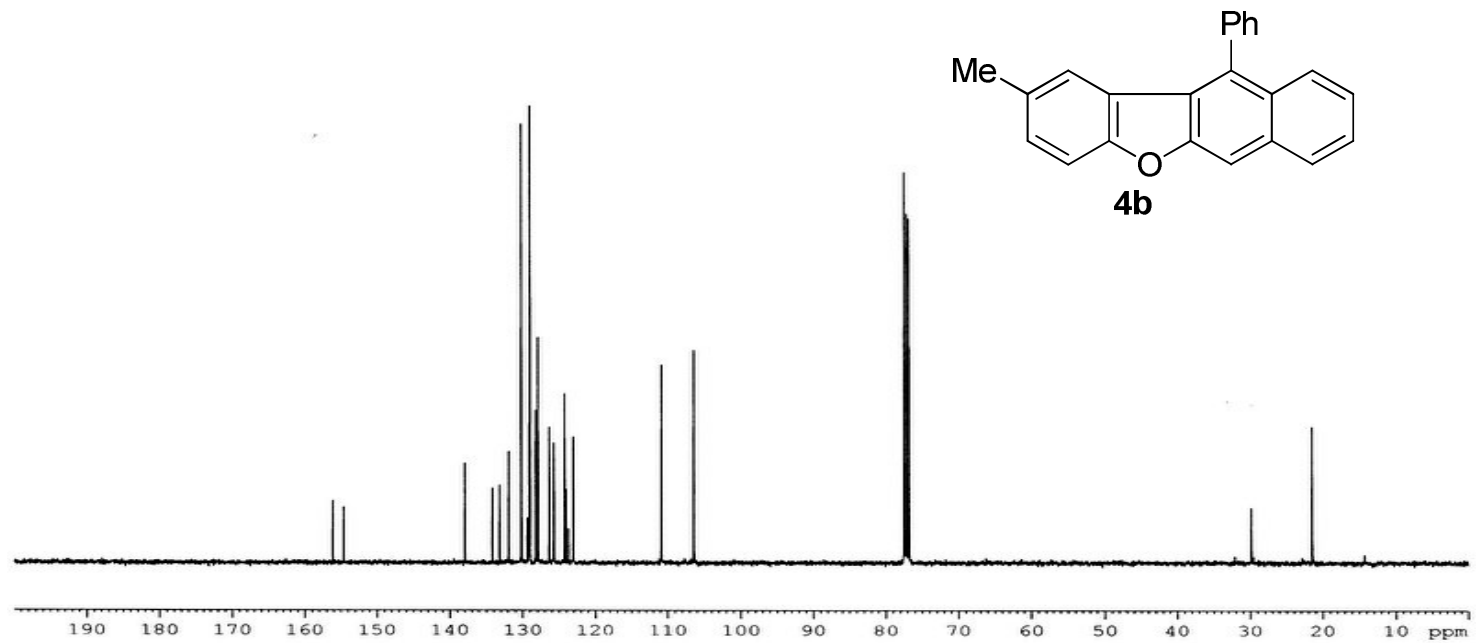




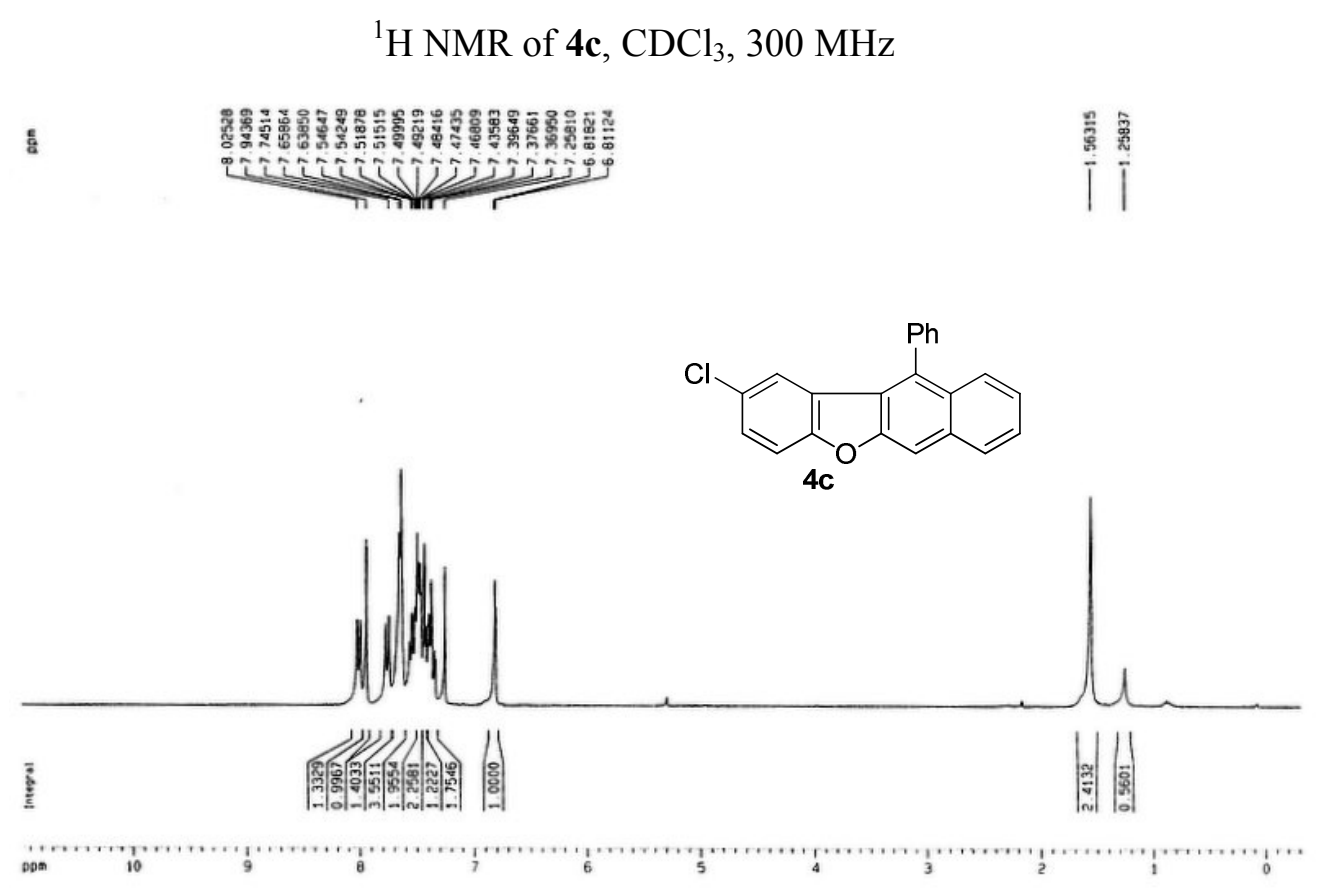

${ }^{13} \mathrm{C} \mathrm{NMR}$ of $4 \mathbf{c}, \mathrm{CDCl}_{3}, 75 \mathrm{MHz}$

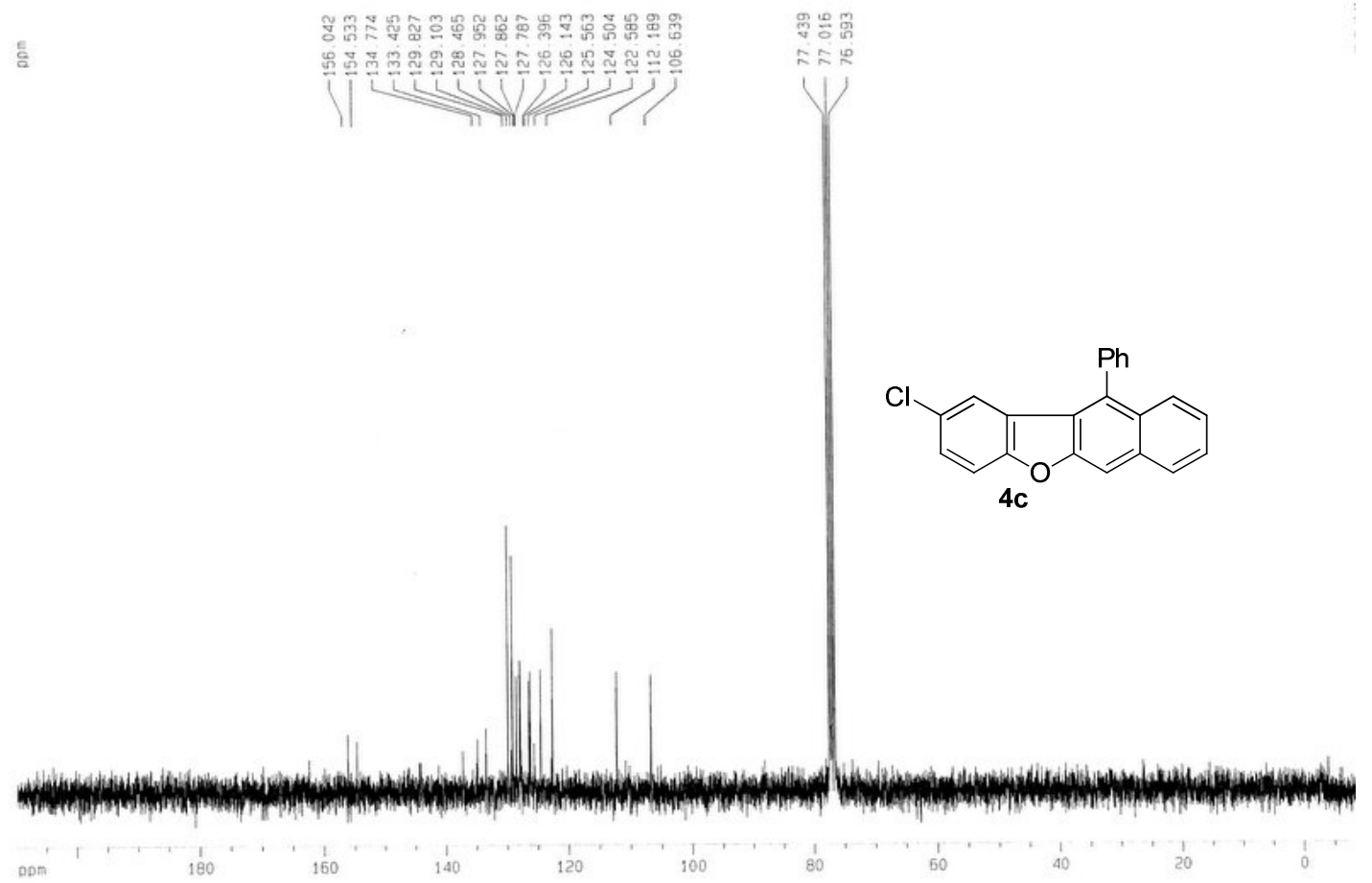


${ }^{1} \mathrm{H}$ NMR of $\mathbf{4 d}, \mathrm{CDCl}_{3}, 300 \mathrm{MHz}$

ฐ

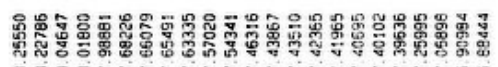

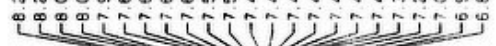

in 11 ir
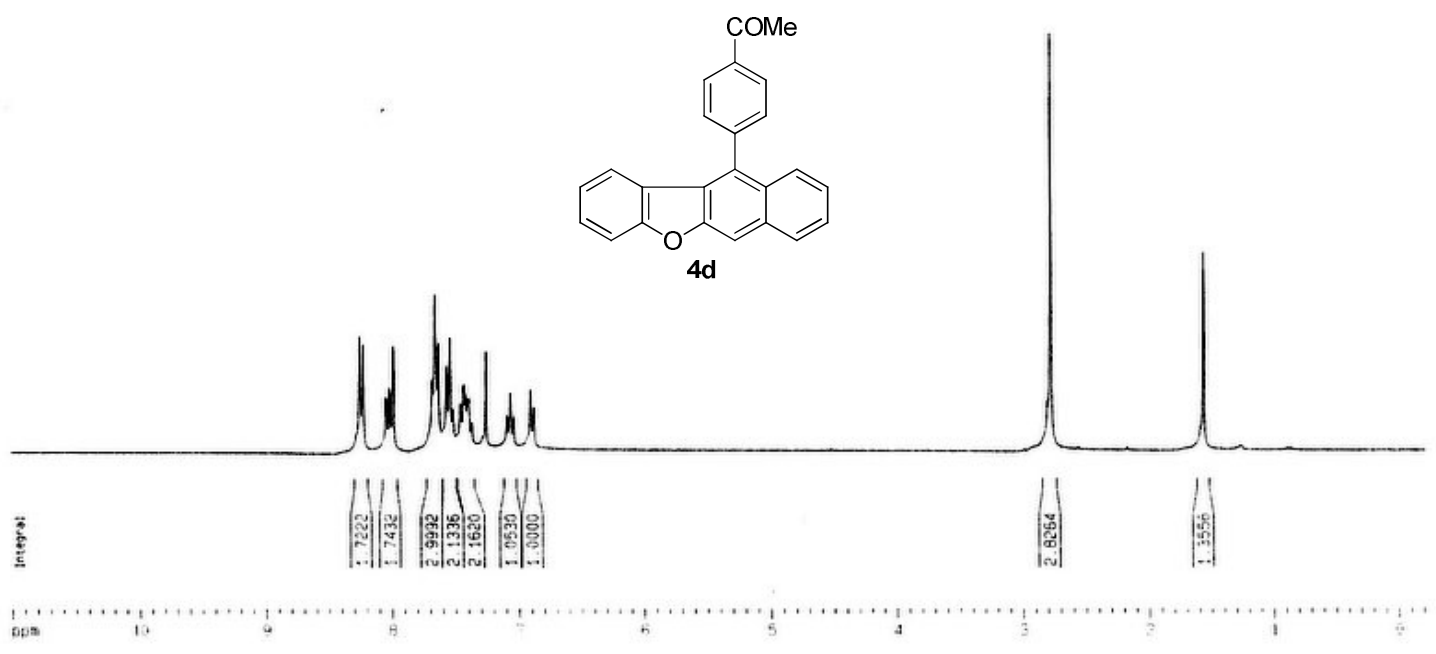

${ }^{13} \mathrm{C} \mathrm{NMR}$ of $\mathbf{4 d}, \mathrm{CDCl}_{3}, 75 \mathrm{MHz}$

5

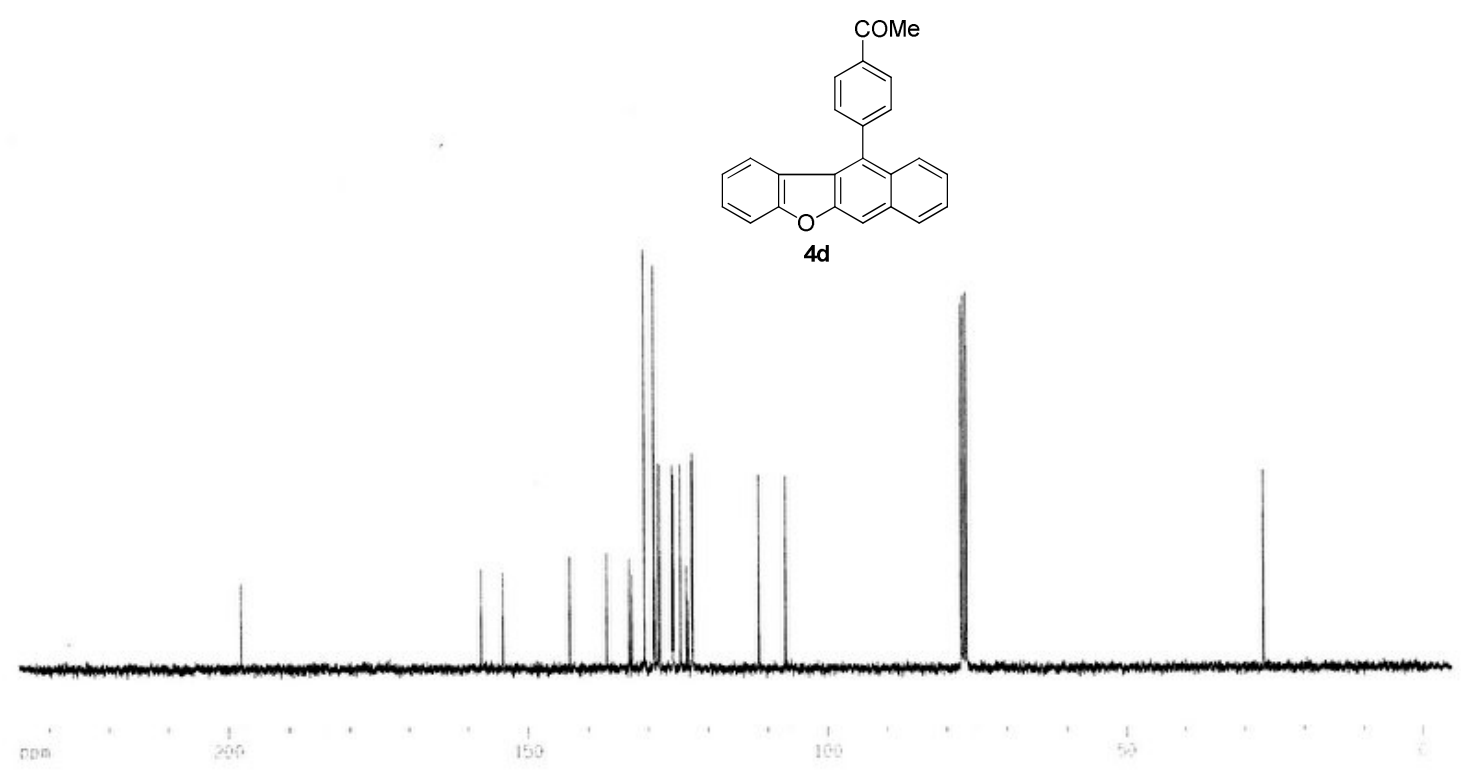


${ }^{1} \mathrm{H} \mathrm{NMR}$ of $4 \mathbf{e}, \mathrm{CDCl}_{3}, 400 \mathrm{MHz}$

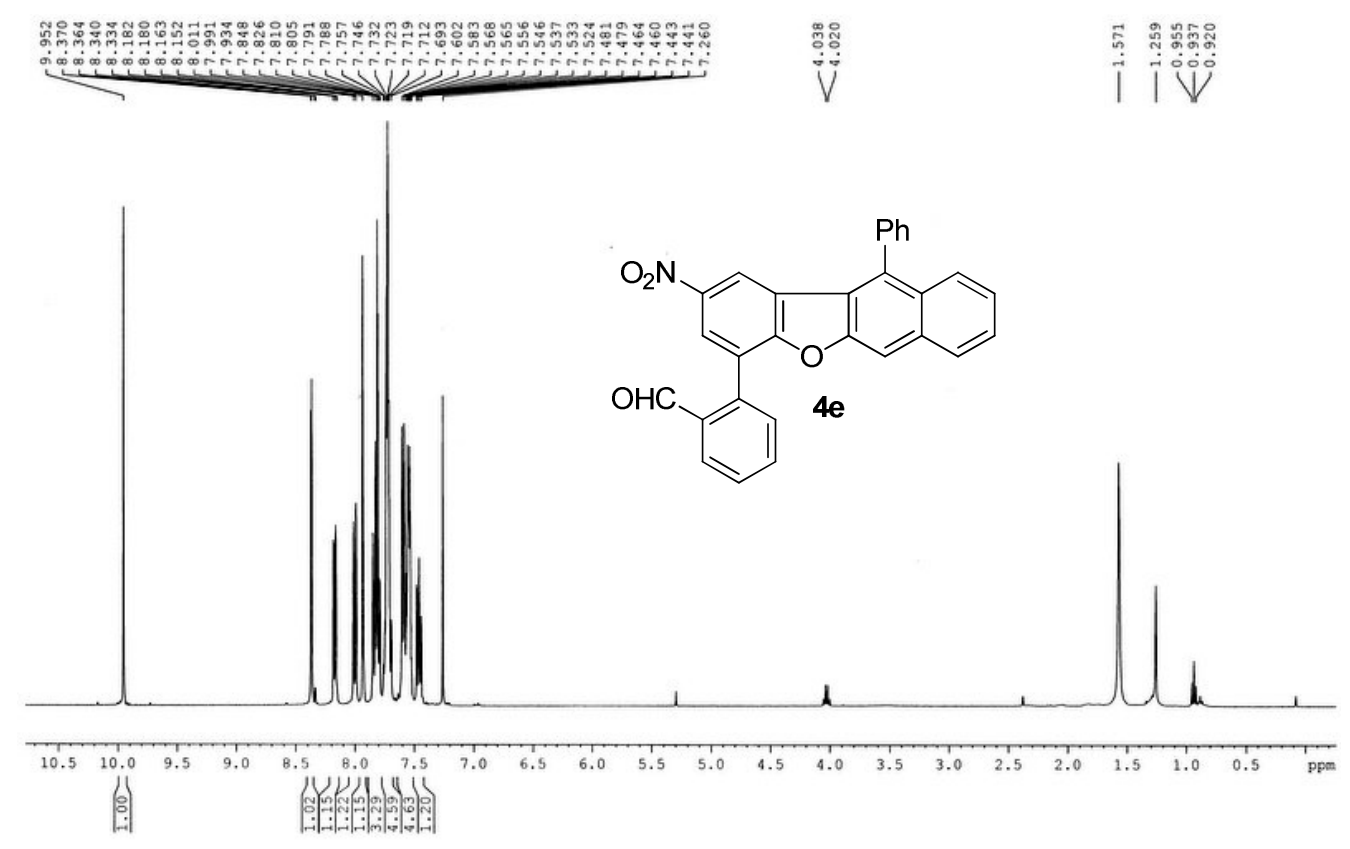

${ }^{13} \mathrm{C} \mathrm{NMR}$ of $4 \mathbf{e}, \mathrm{CDCl}_{3}, 75 \mathrm{MHz}$

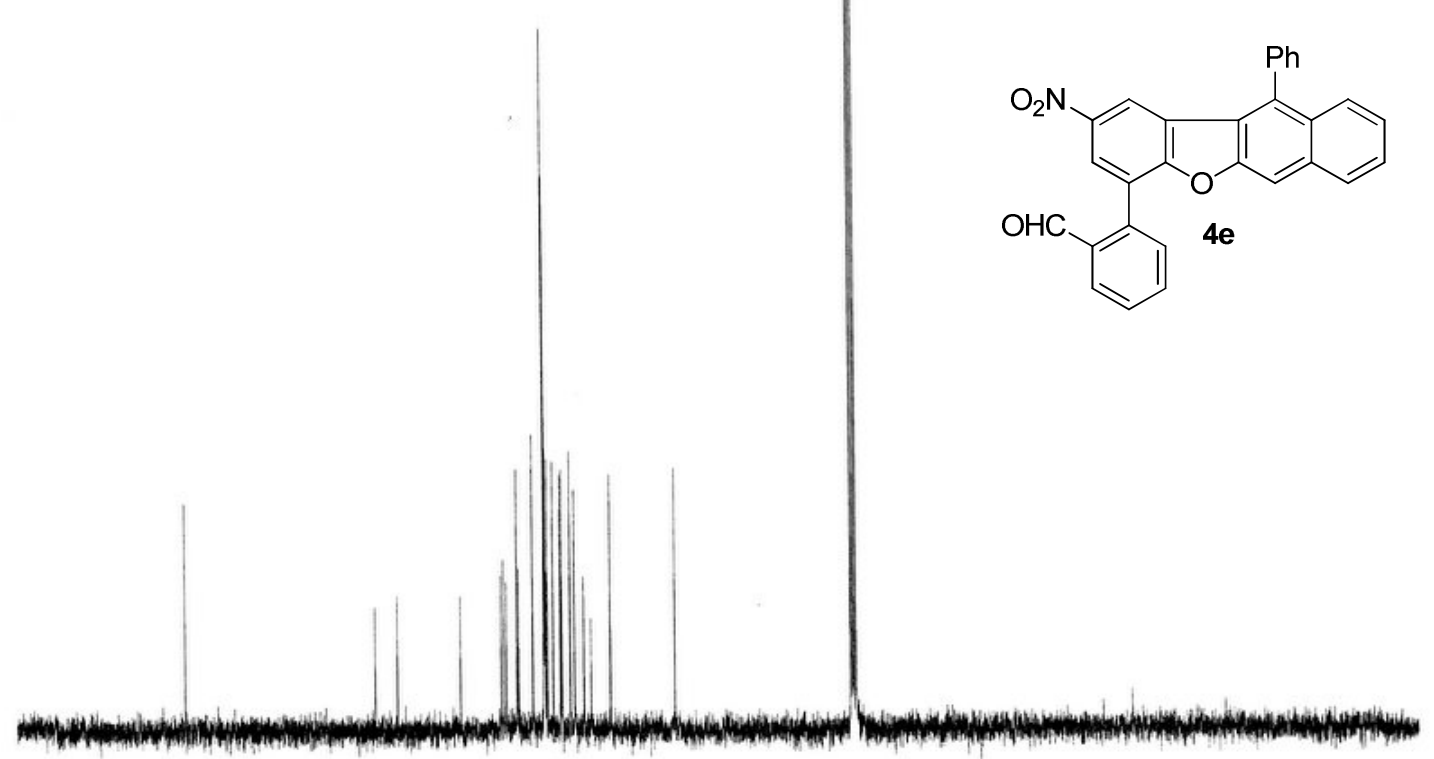




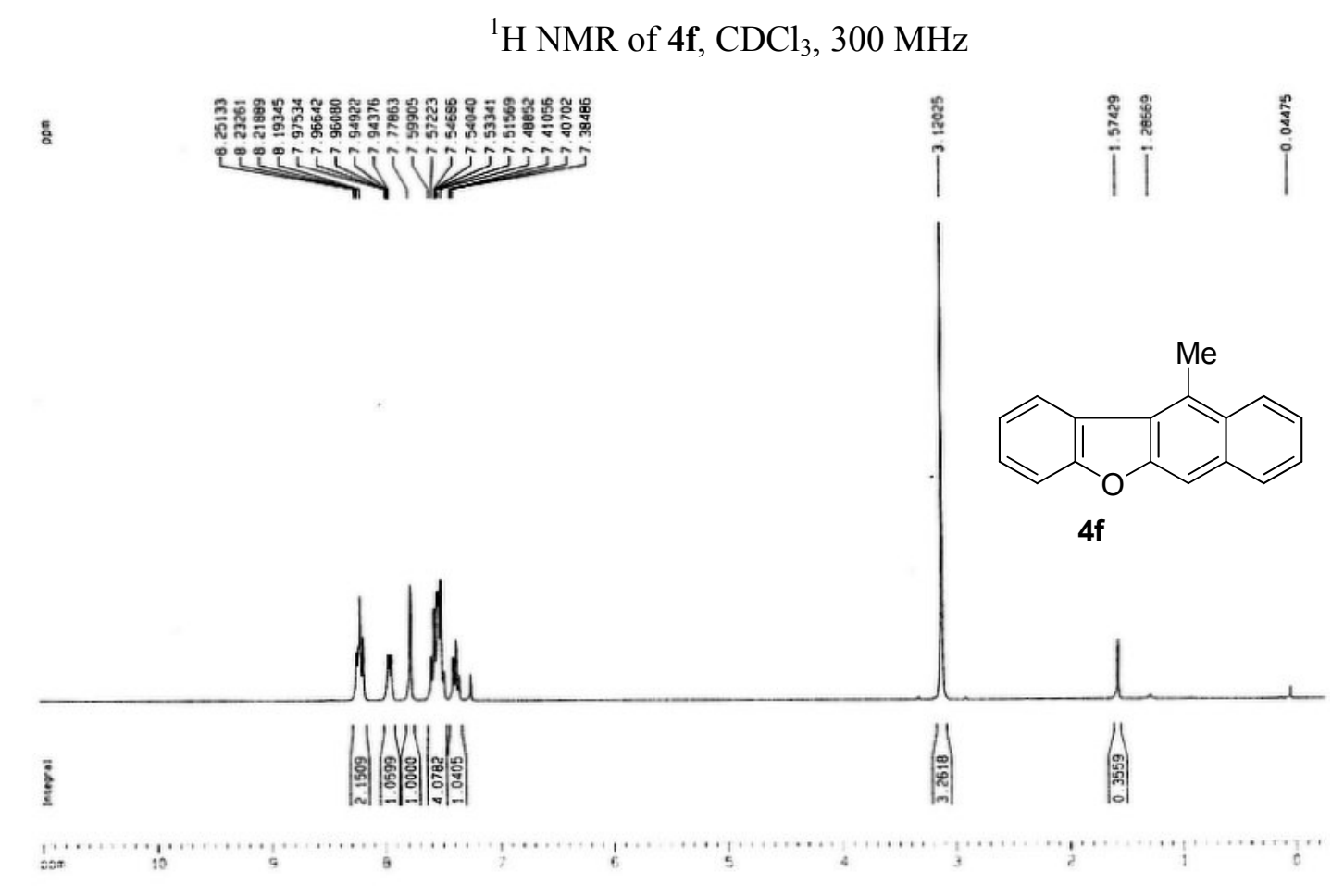

${ }^{13} \mathrm{C}$ NMR of $4 \mathbf{f}, \mathrm{CDCl}_{3}, 75 \mathrm{MHz}$

言

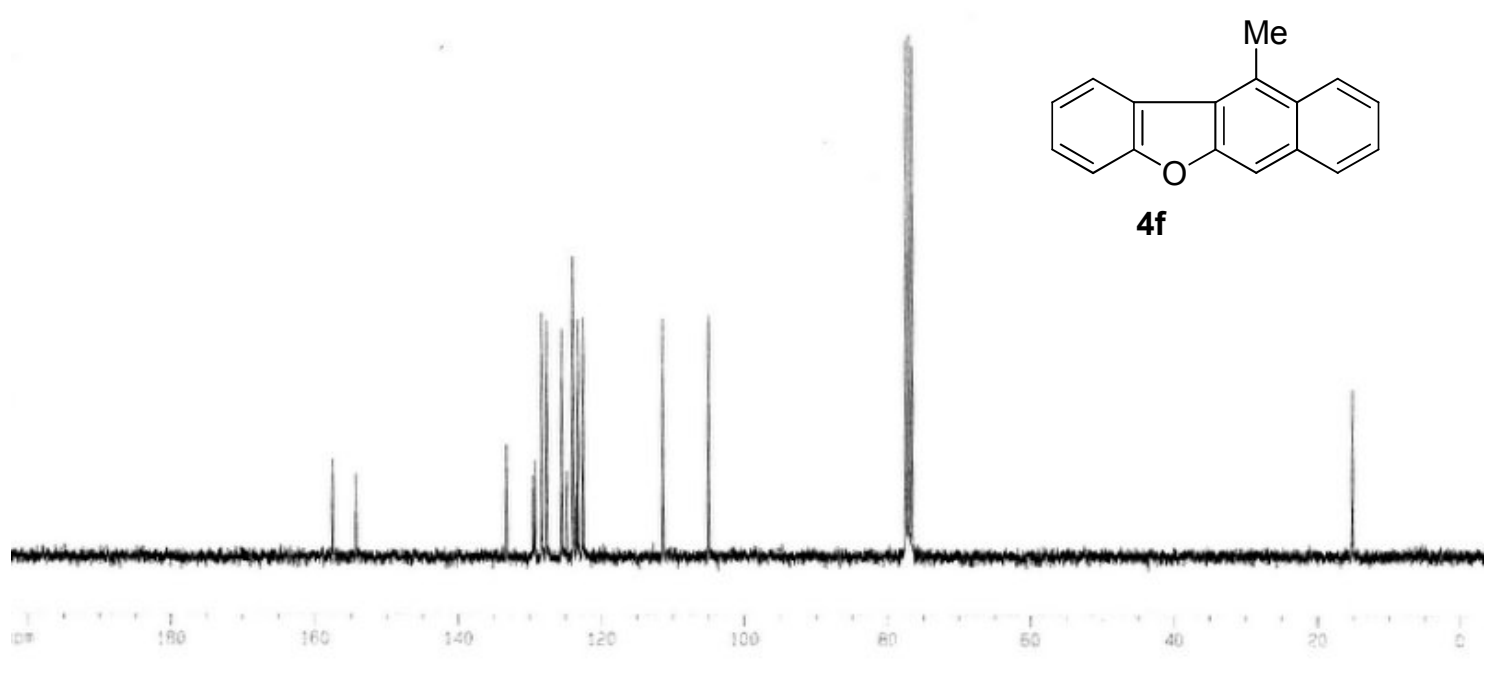


${ }^{1} \mathrm{H}$ NMR of $\mathbf{4 g}, \mathrm{CDCl}_{3}, 300 \mathrm{MHz}$

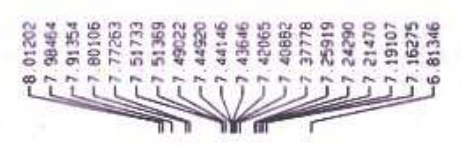

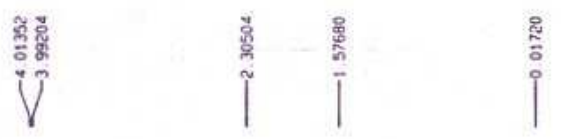

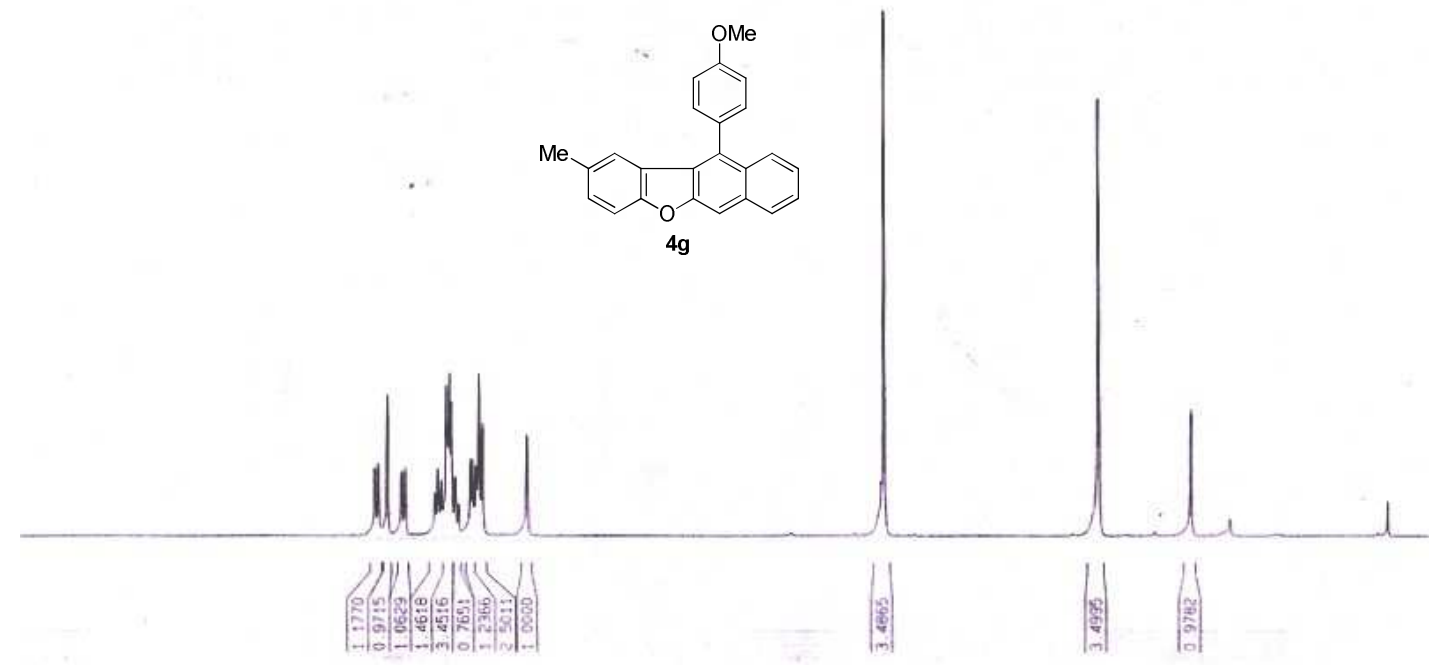

${ }^{13} \mathrm{C} \mathrm{NMR}$ of $4 \mathrm{~g}, \mathrm{CDCl}_{3}, 75 \mathrm{MHz}$
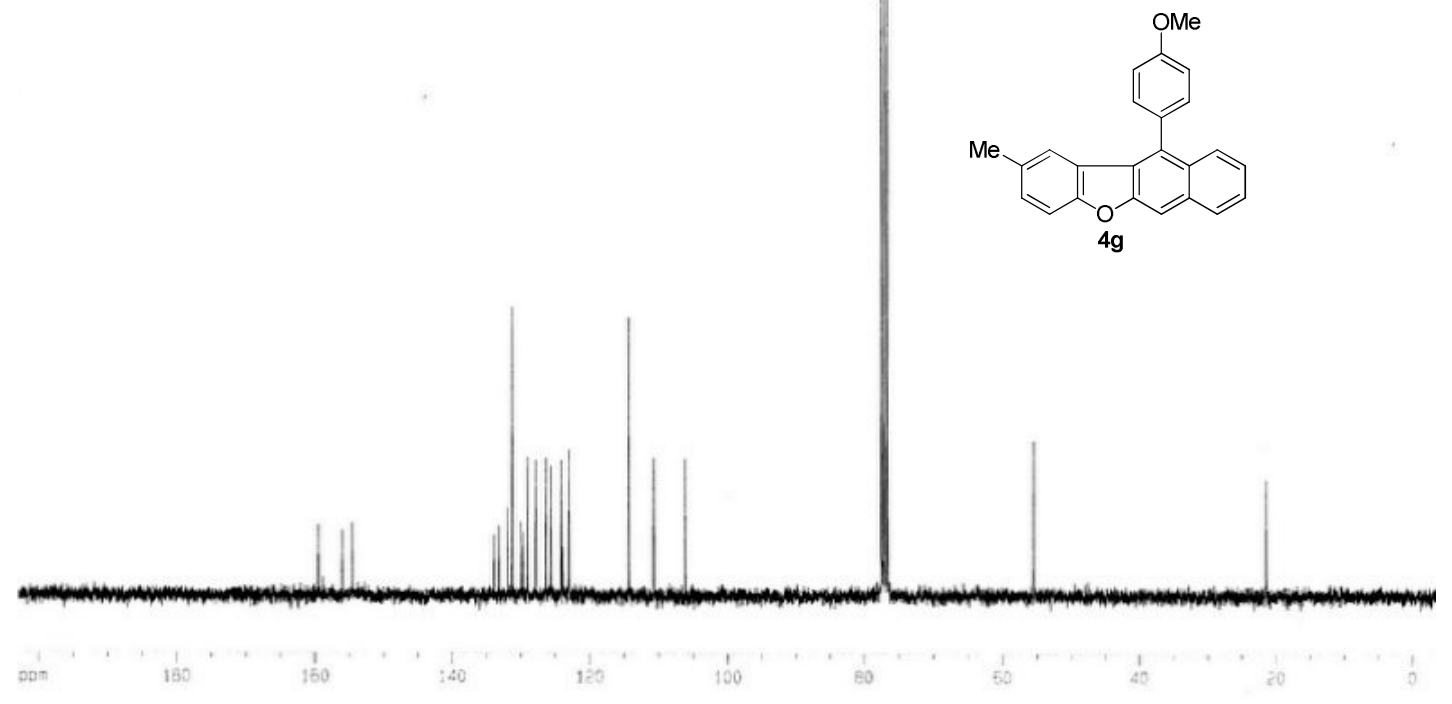
${ }^{1} \mathrm{H}$ NMR of $\mathbf{4 h}, \mathrm{CDCl}_{3}, 300 \mathrm{MHz}$

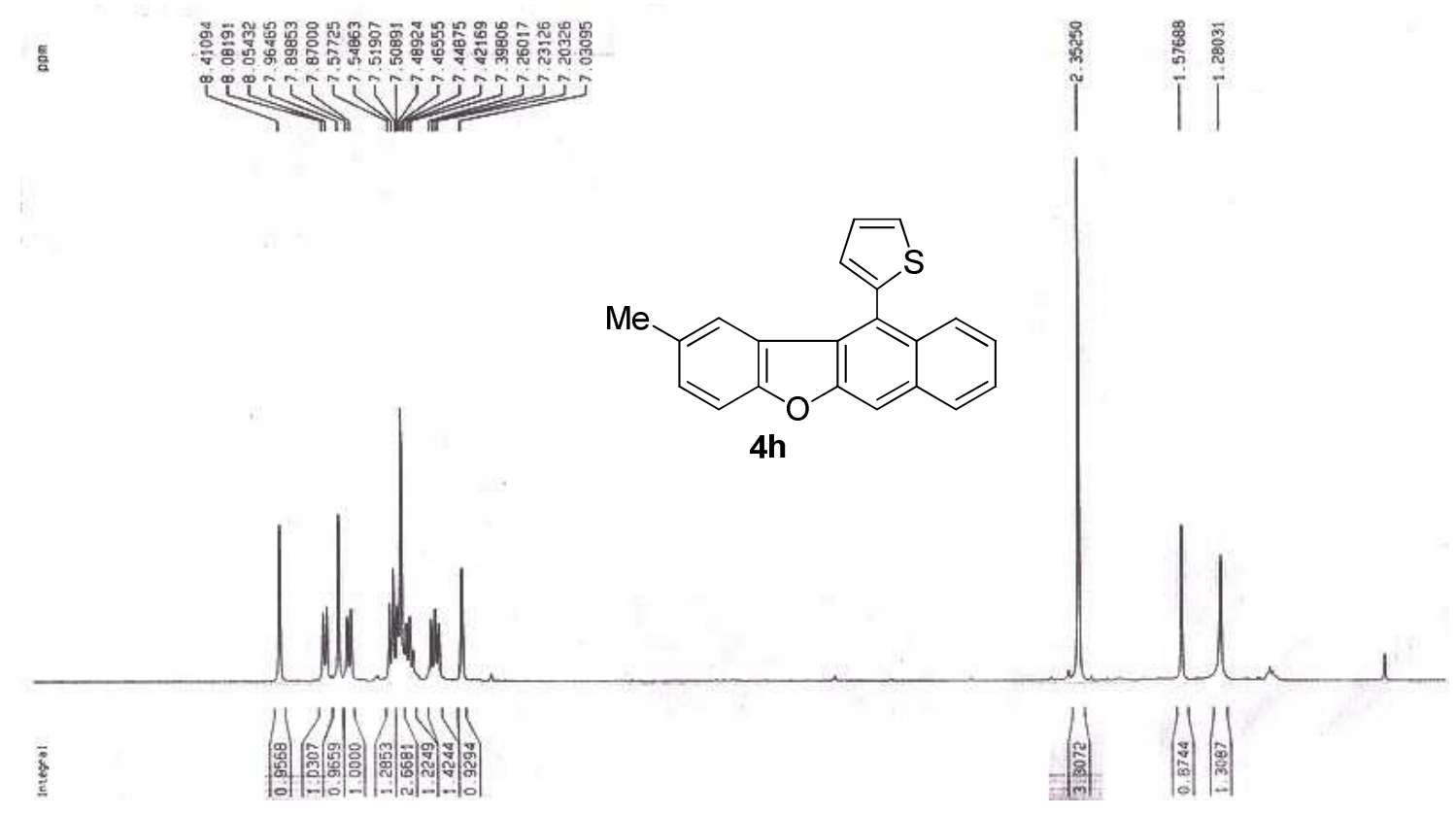

${ }^{13} \mathrm{C}$ NMR of $\mathbf{4 h}, \mathrm{CDCl}_{3} 300 \mathrm{MHz}$

站
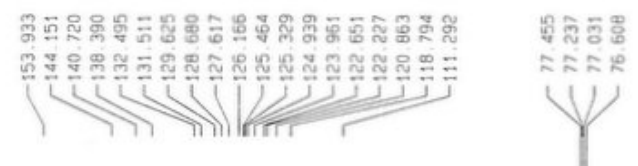

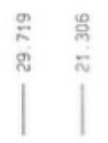<smiles>COc1ccc2oc3cc4ccccc4c(-c4cccs4)c3c2c1</smiles>

4h 
${ }^{1} \mathrm{H}$ NMR of 4i, $\mathrm{CDCl}_{3} 300 \mathrm{MHz}$

ธ
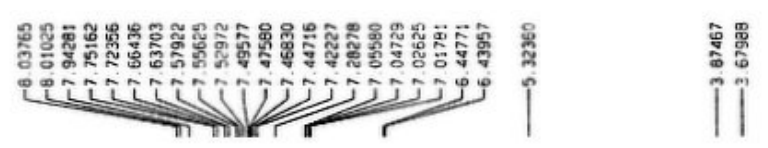

广

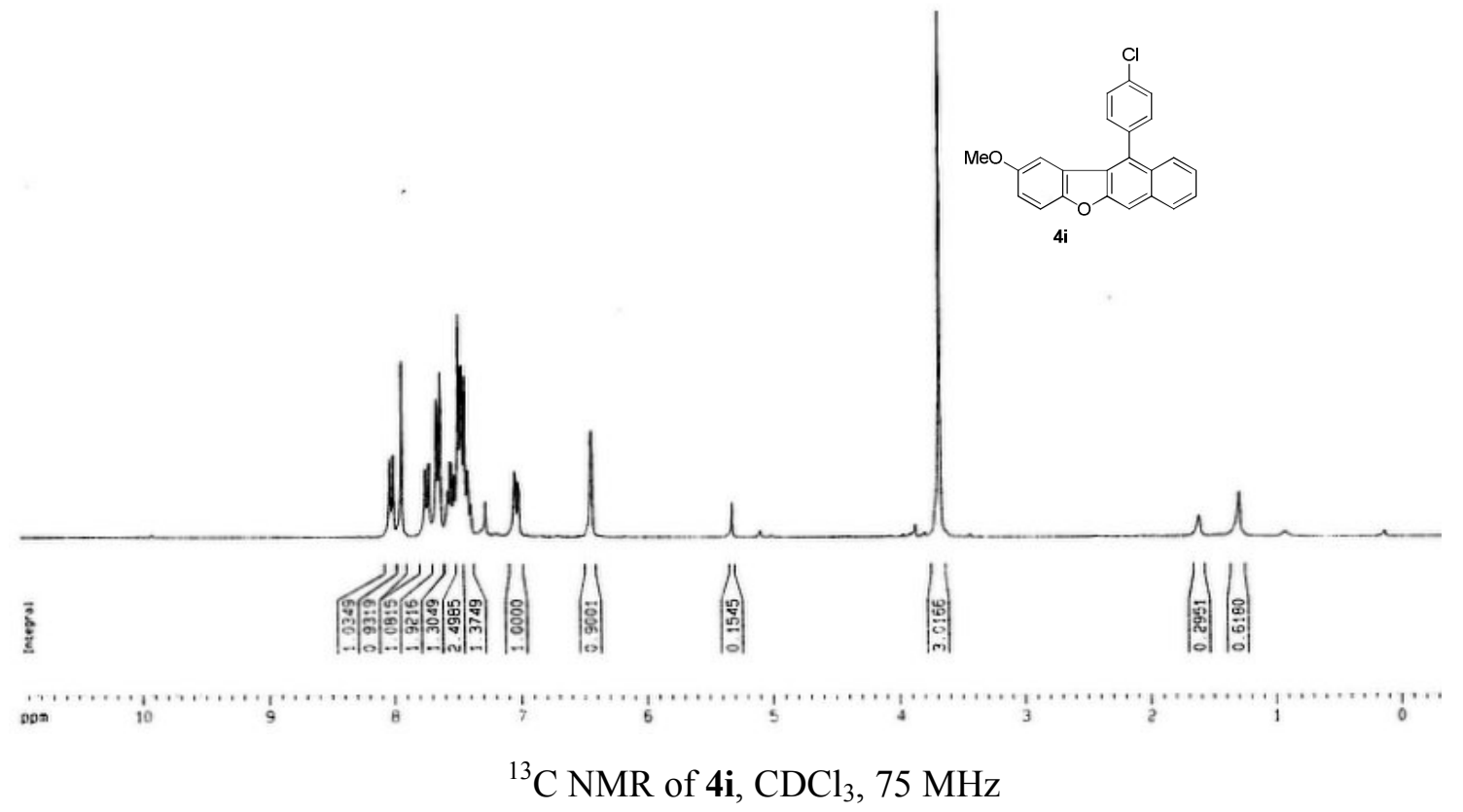

镸

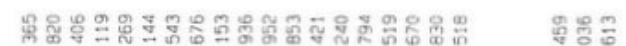

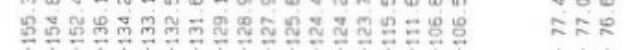

vilutiry

V

|

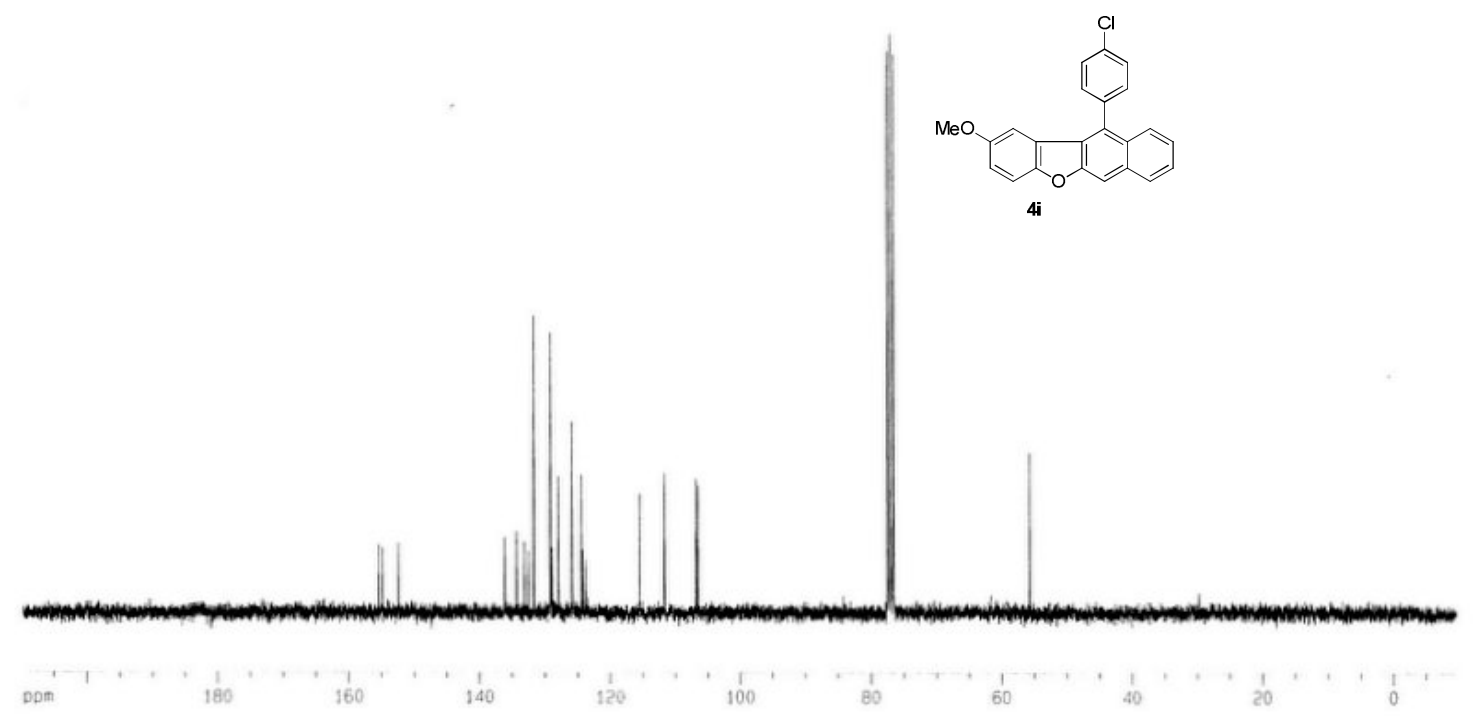


${ }^{1} \mathrm{H}$ NMR of $\mathbf{4 j}, \mathrm{CDCl}_{3}, 300 \mathrm{MHz}$

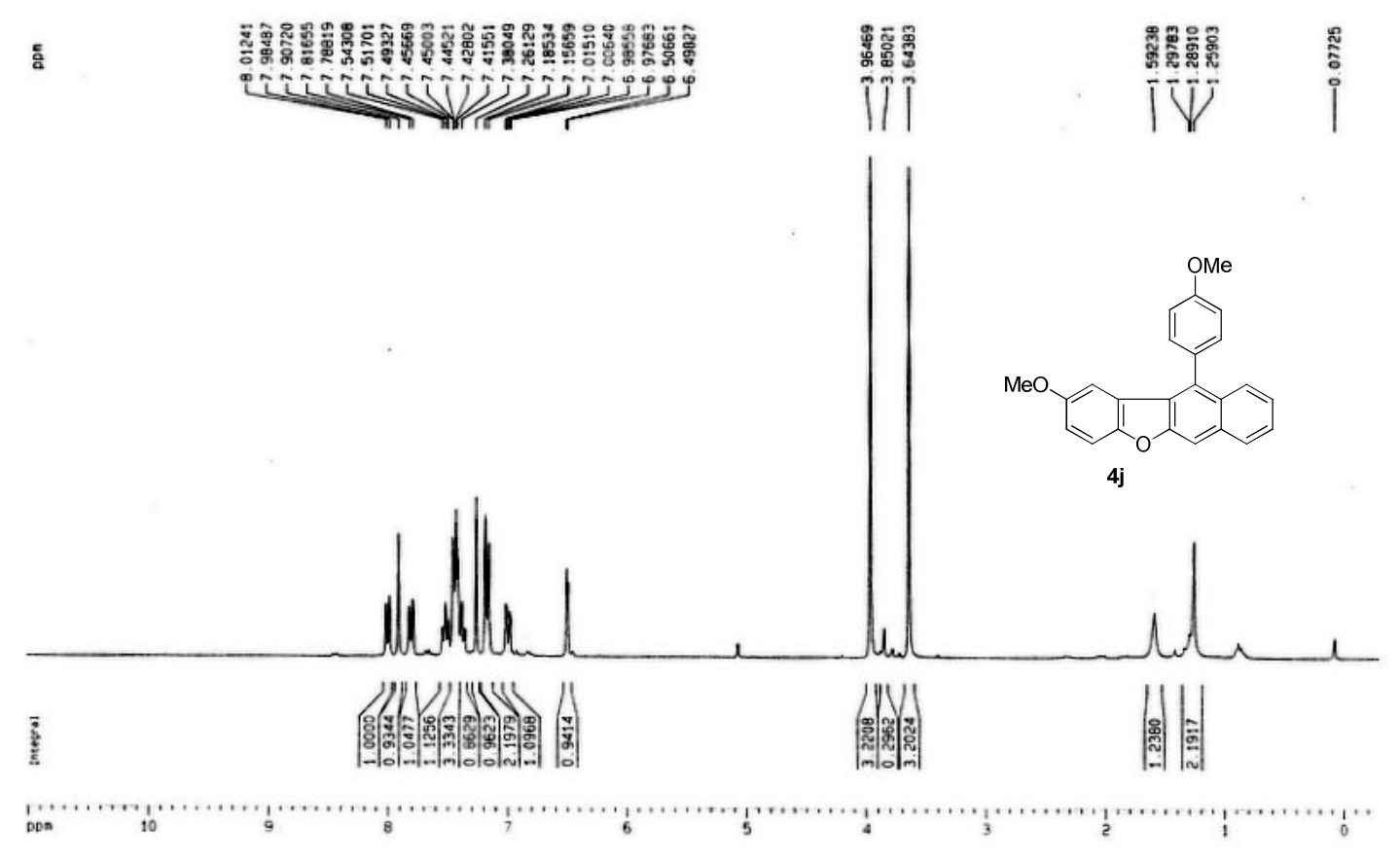

${ }^{13} \mathrm{C} \mathrm{NMR}$ of $4 \mathbf{j}, \mathrm{CDCl}_{3}, 75 \mathrm{MHz}$

ă

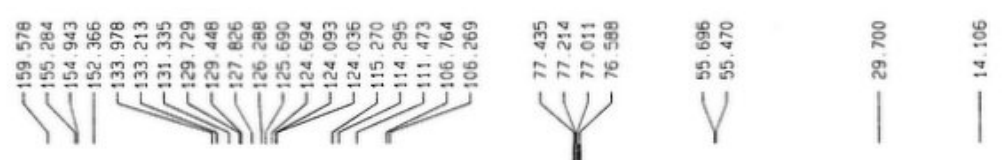

SUl

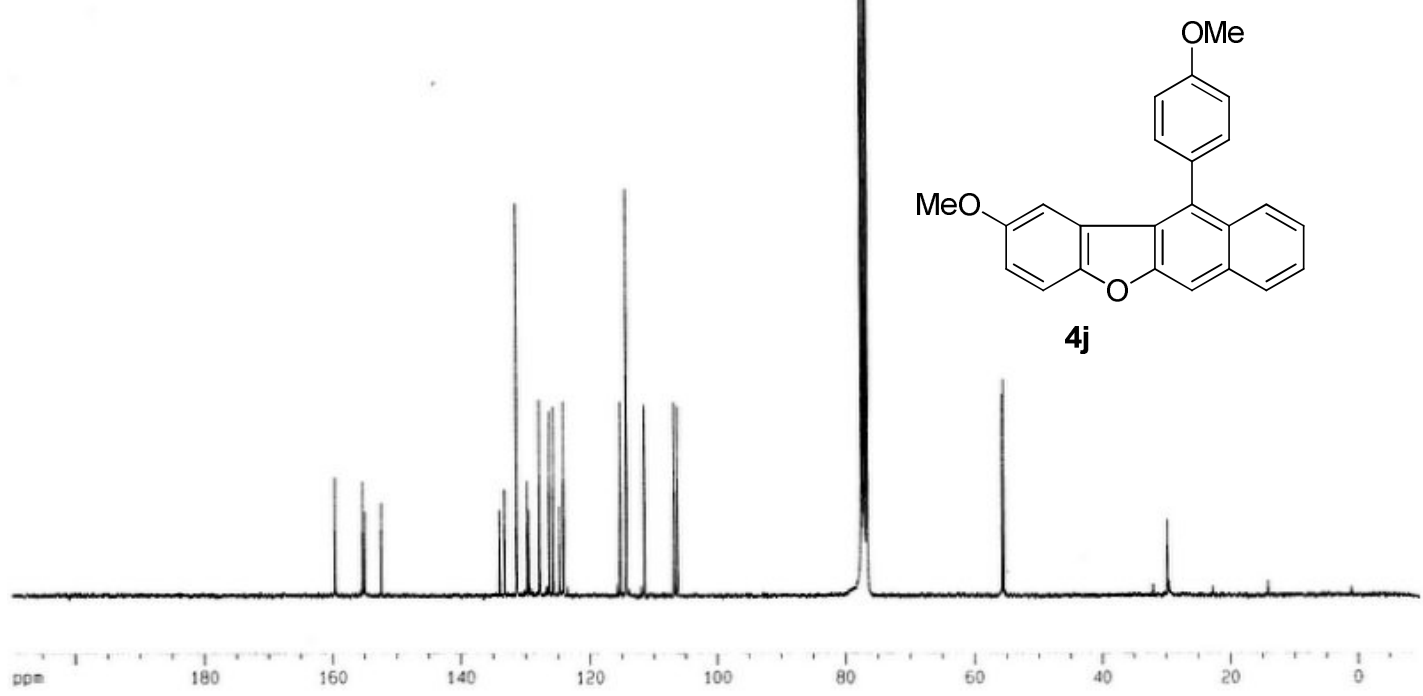



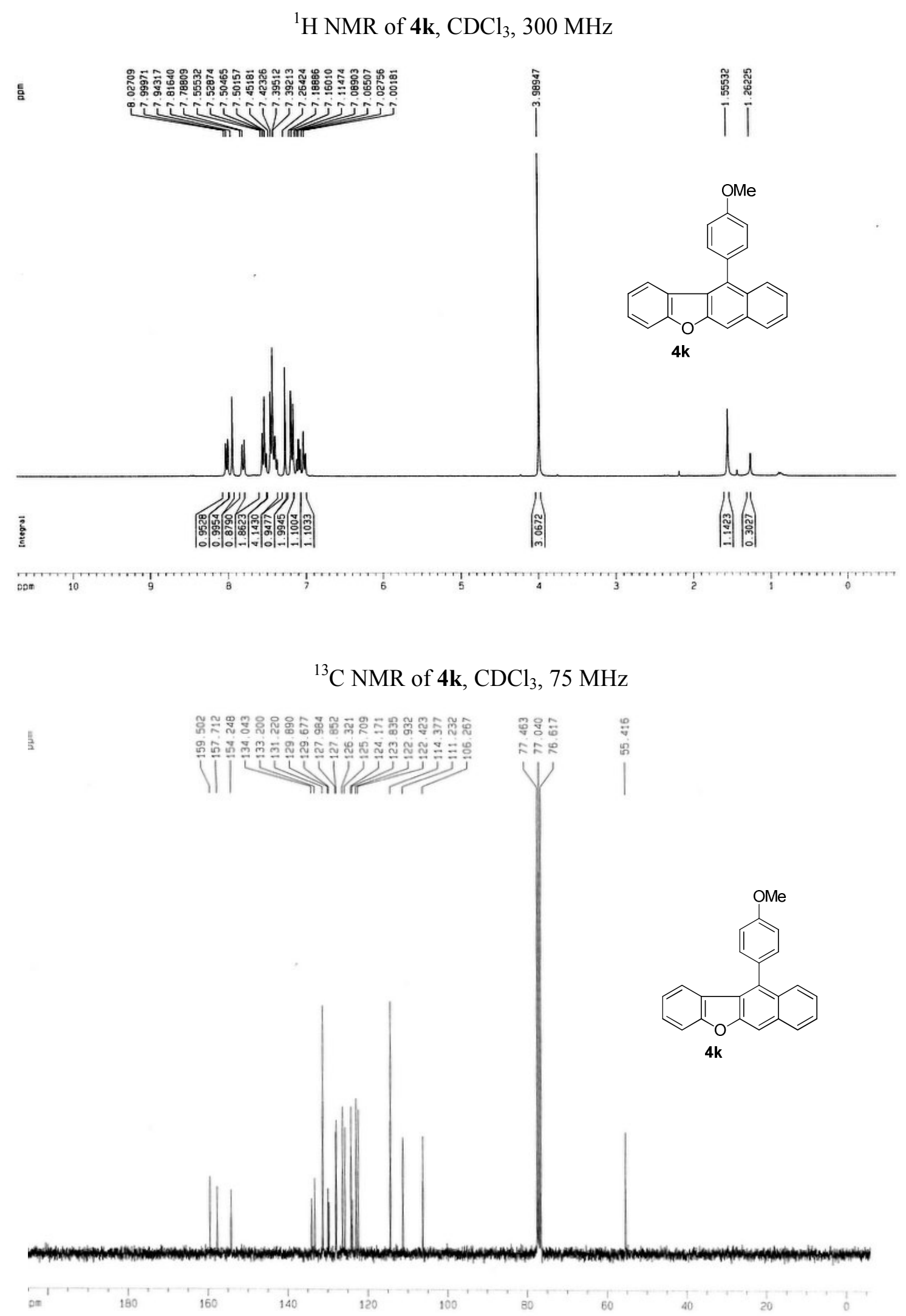
${ }^{1} \mathrm{H} \mathrm{NMR} \mathrm{of} \mathrm{4l,} \mathrm{CDCl}_{3}, 300 \mathrm{MHz}$

ธิ

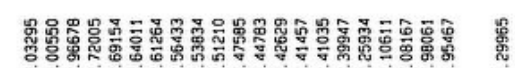

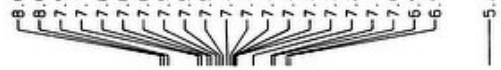

$\mid$

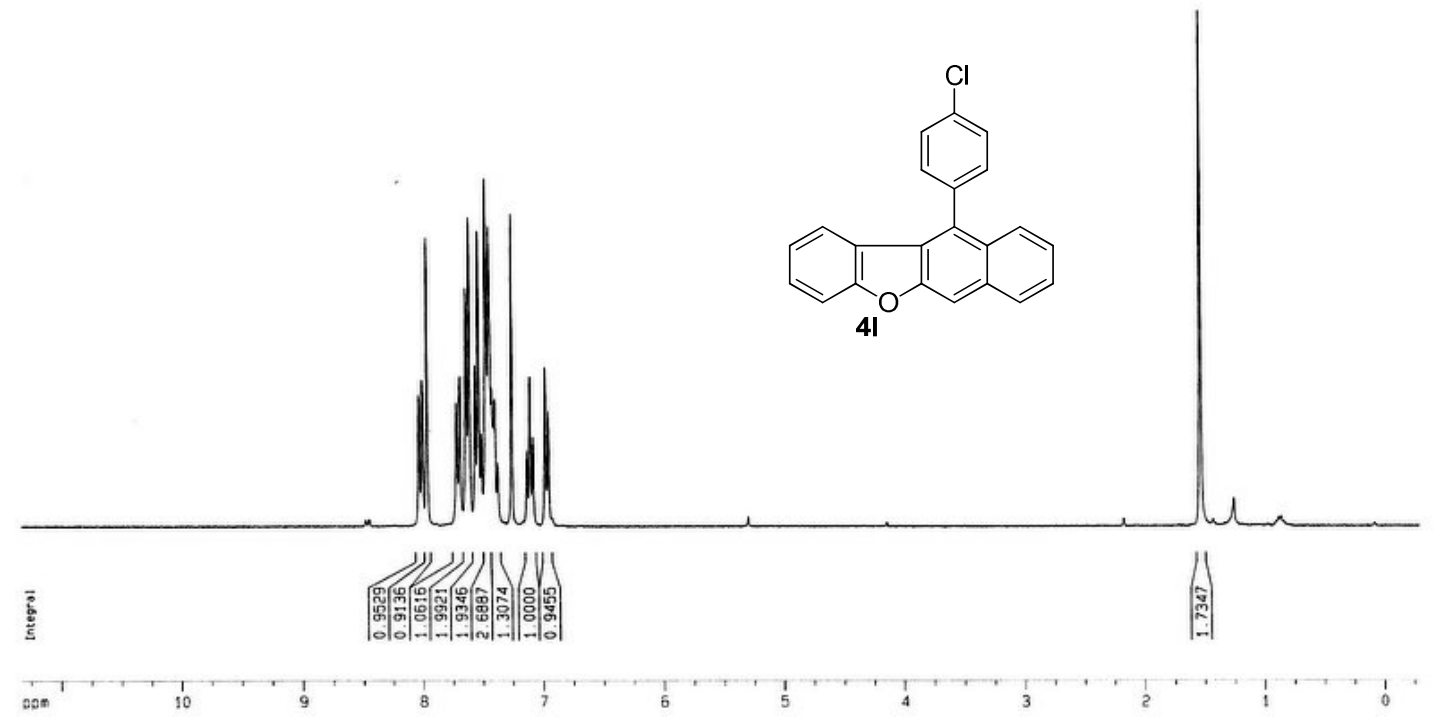

${ }^{13} \mathrm{C}$ NMR of $4 \mathbf{l}, \mathrm{CDCl}_{3}, 75 \mathrm{MHz}$

⿷匚⿱⿰㇒一大口

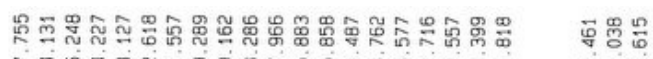

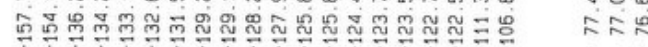

1hin
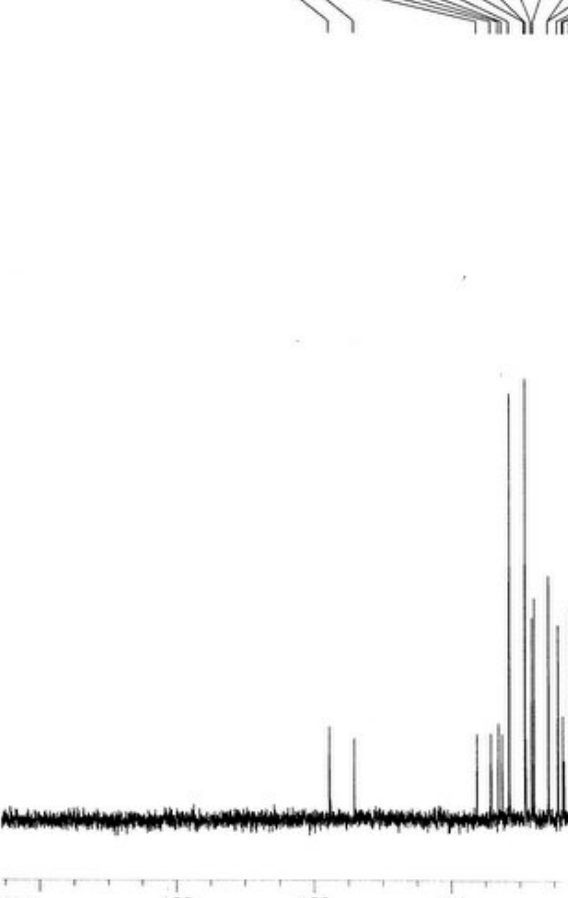

$\mathrm{ppm}$

160

140

120
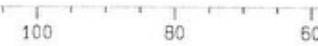

60

40 
${ }^{1} \mathrm{H} \mathrm{NMR}$ of $\mathbf{4 m}, \mathrm{CDCl}_{3}, 300 \mathrm{MHz}$

s

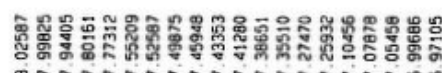

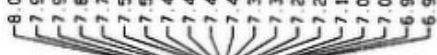
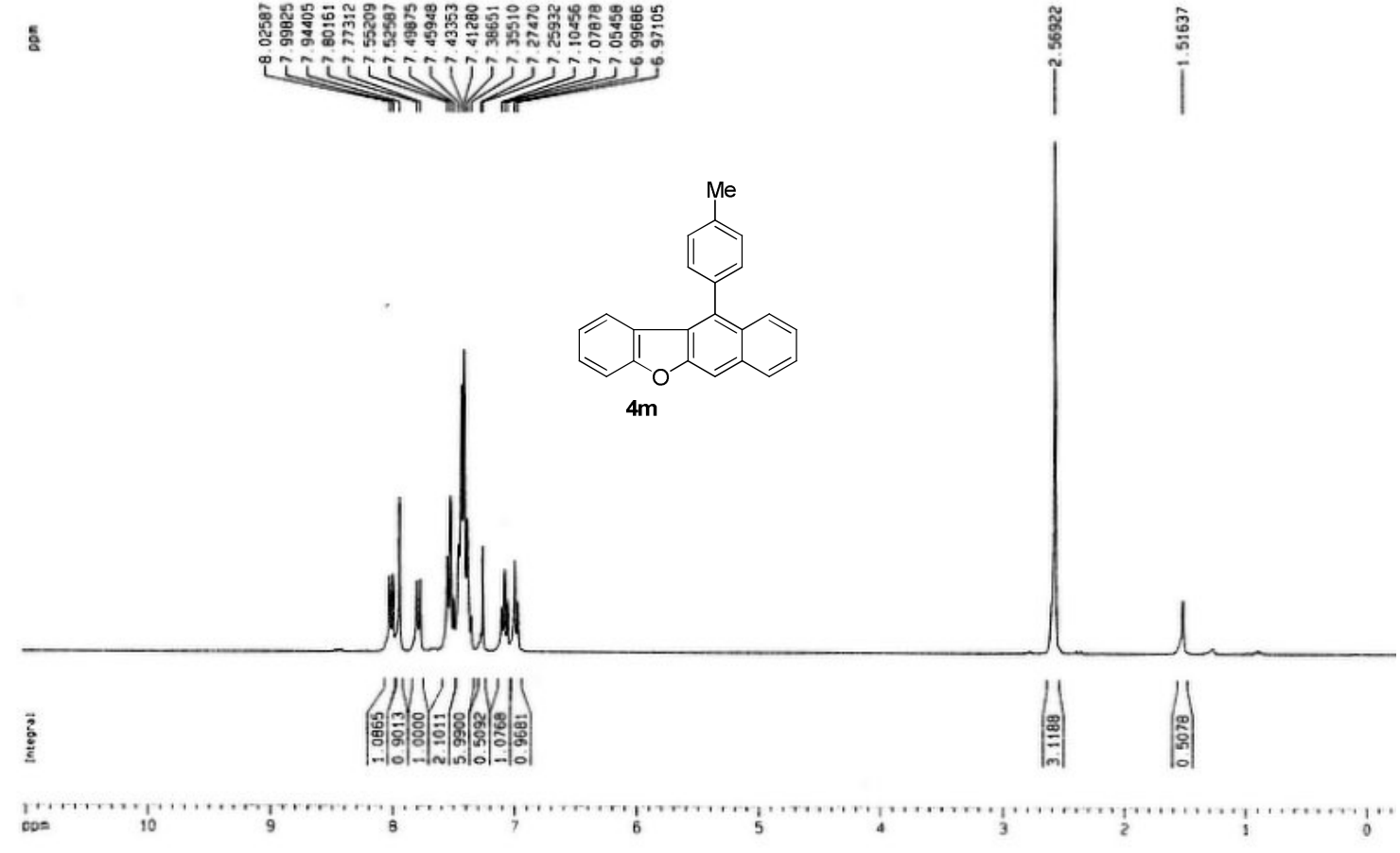

${ }^{13} \mathrm{C}$ NMR of $4 \mathbf{m}, \mathrm{CDCl}_{3}, 75 \mathrm{MHz}$

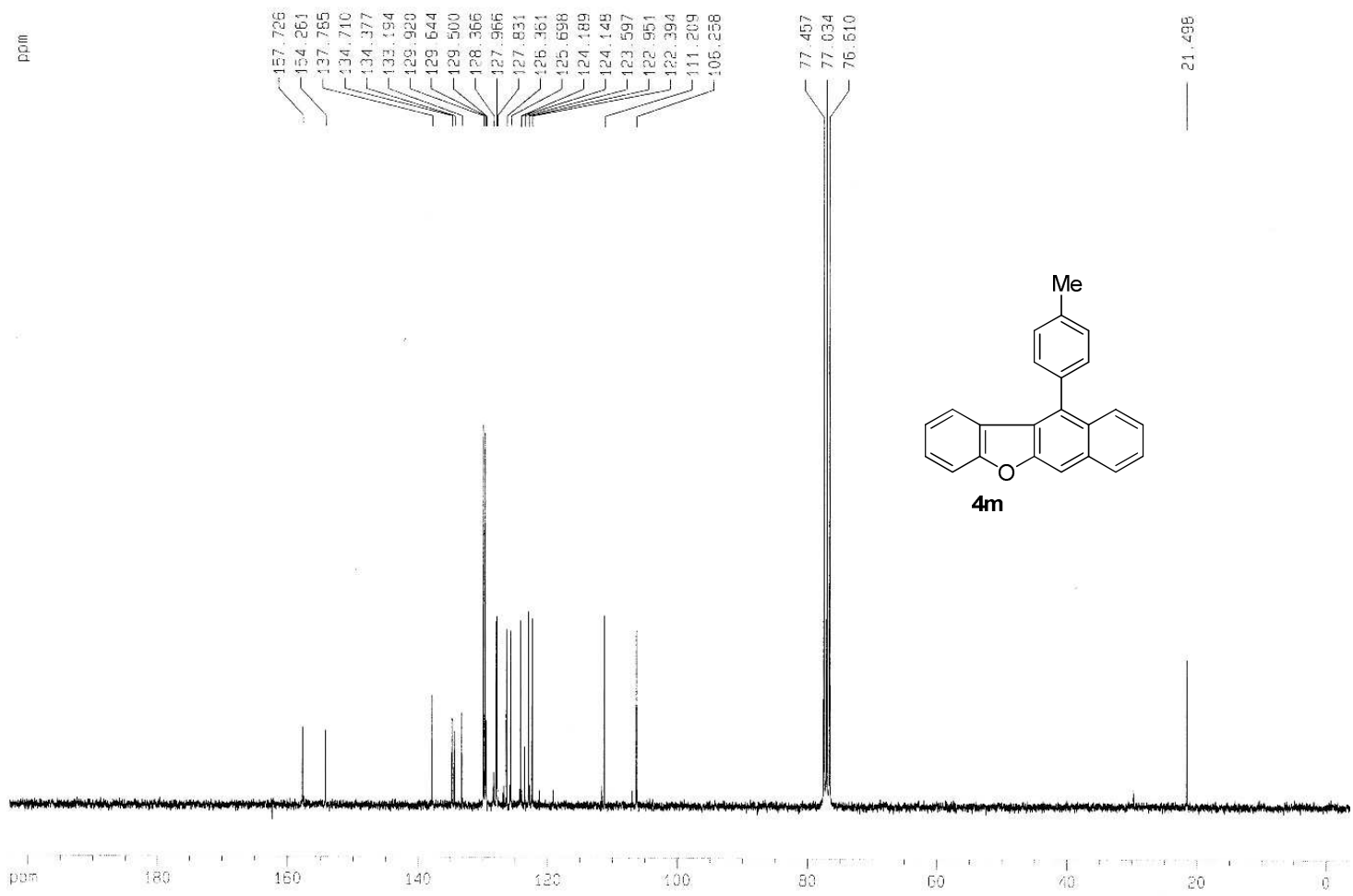


${ }^{1} \mathrm{H}$ NMR of $4 \mathbf{n}, \mathrm{CDCl}_{3}, 300 \mathrm{MHz}$

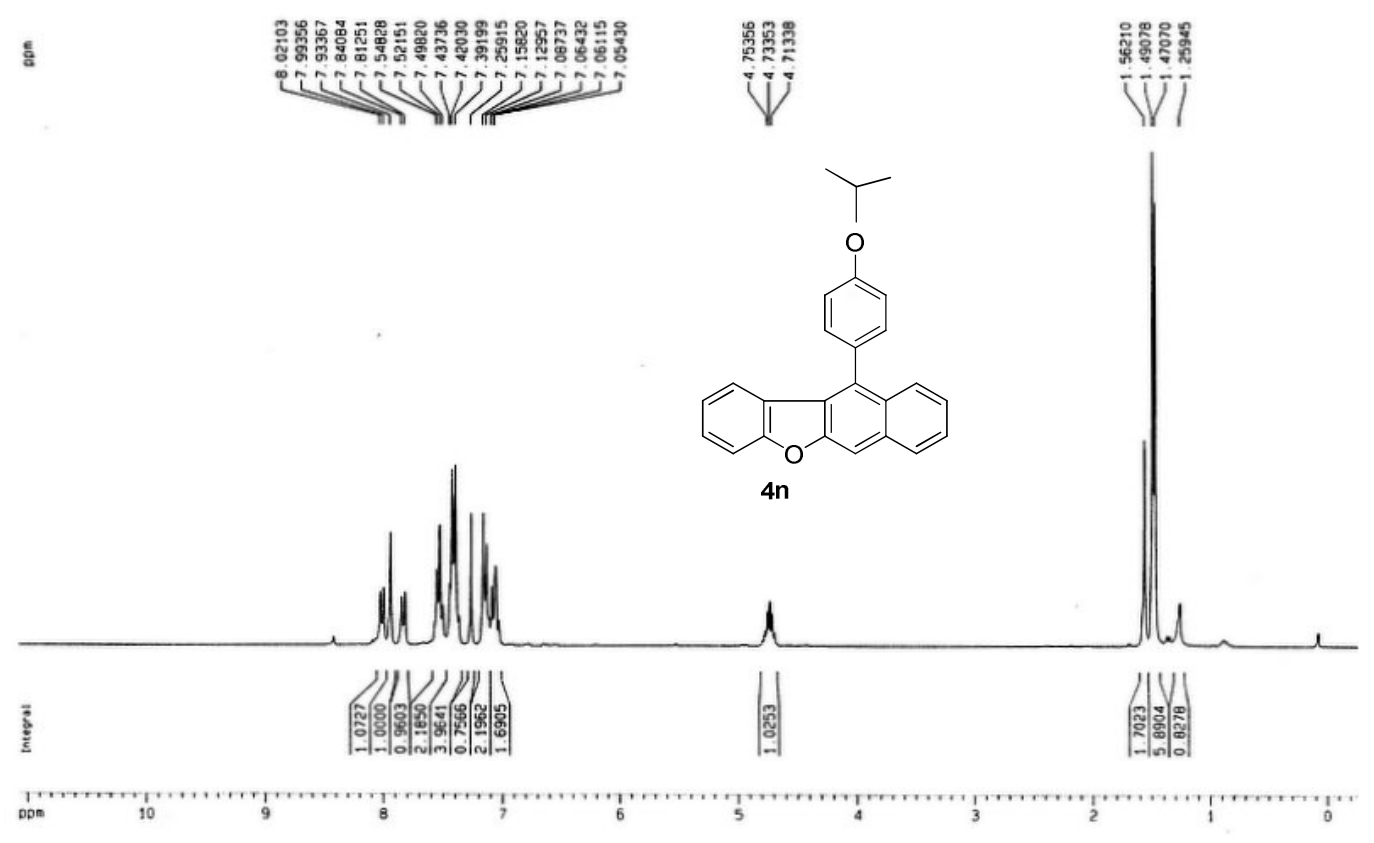

${ }^{13} \mathrm{C} \mathrm{NMR}$ of $4 \mathbf{n}, \mathrm{CDCl}_{3}, 75 \mathrm{MHz}$

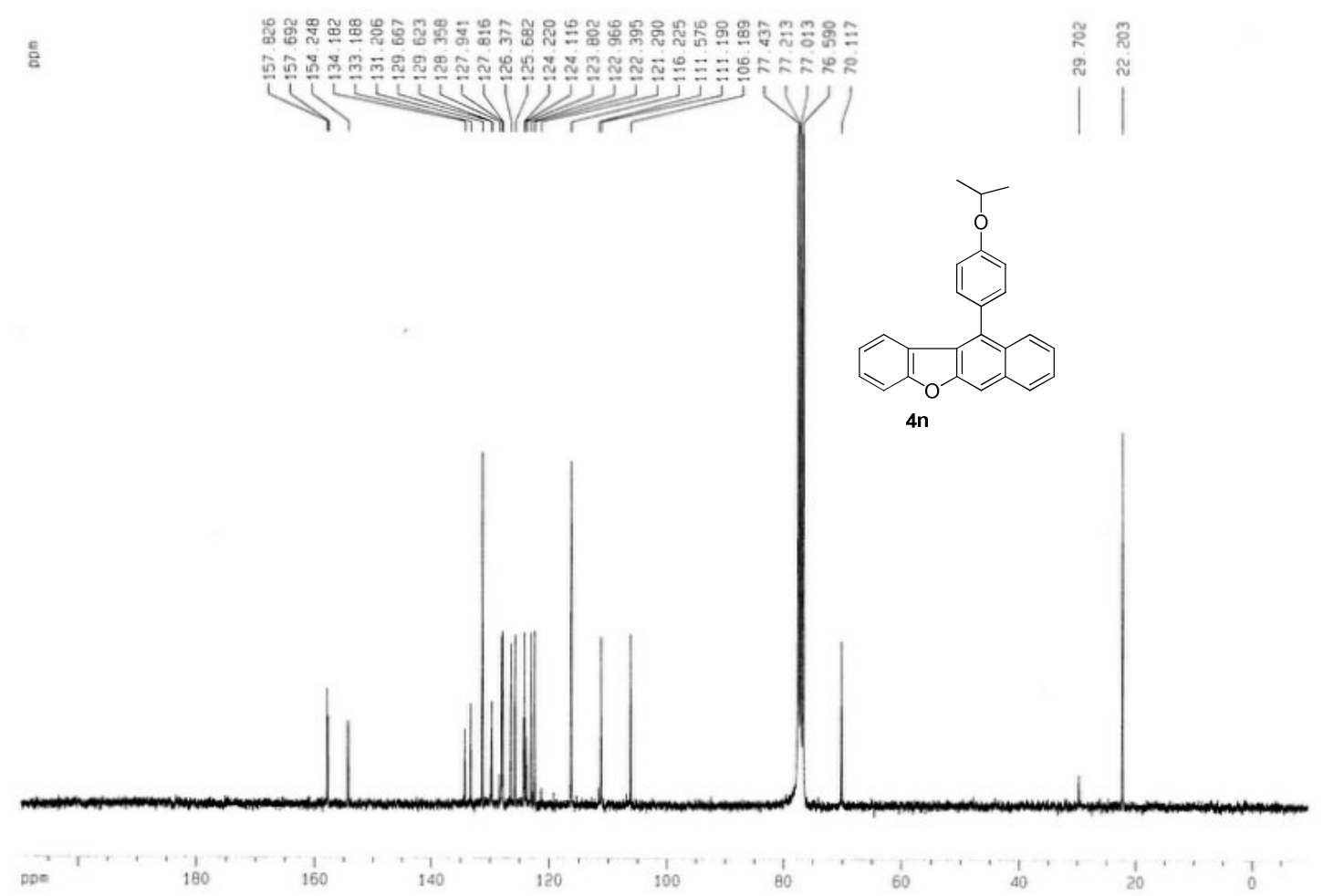


${ }^{1} \mathrm{H}$ NMR of $4 \mathbf{4 o}, \mathrm{CDCl}_{3}, 300 \mathrm{MHz}$

s

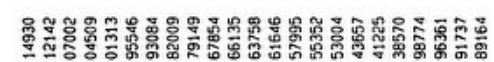

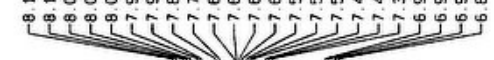
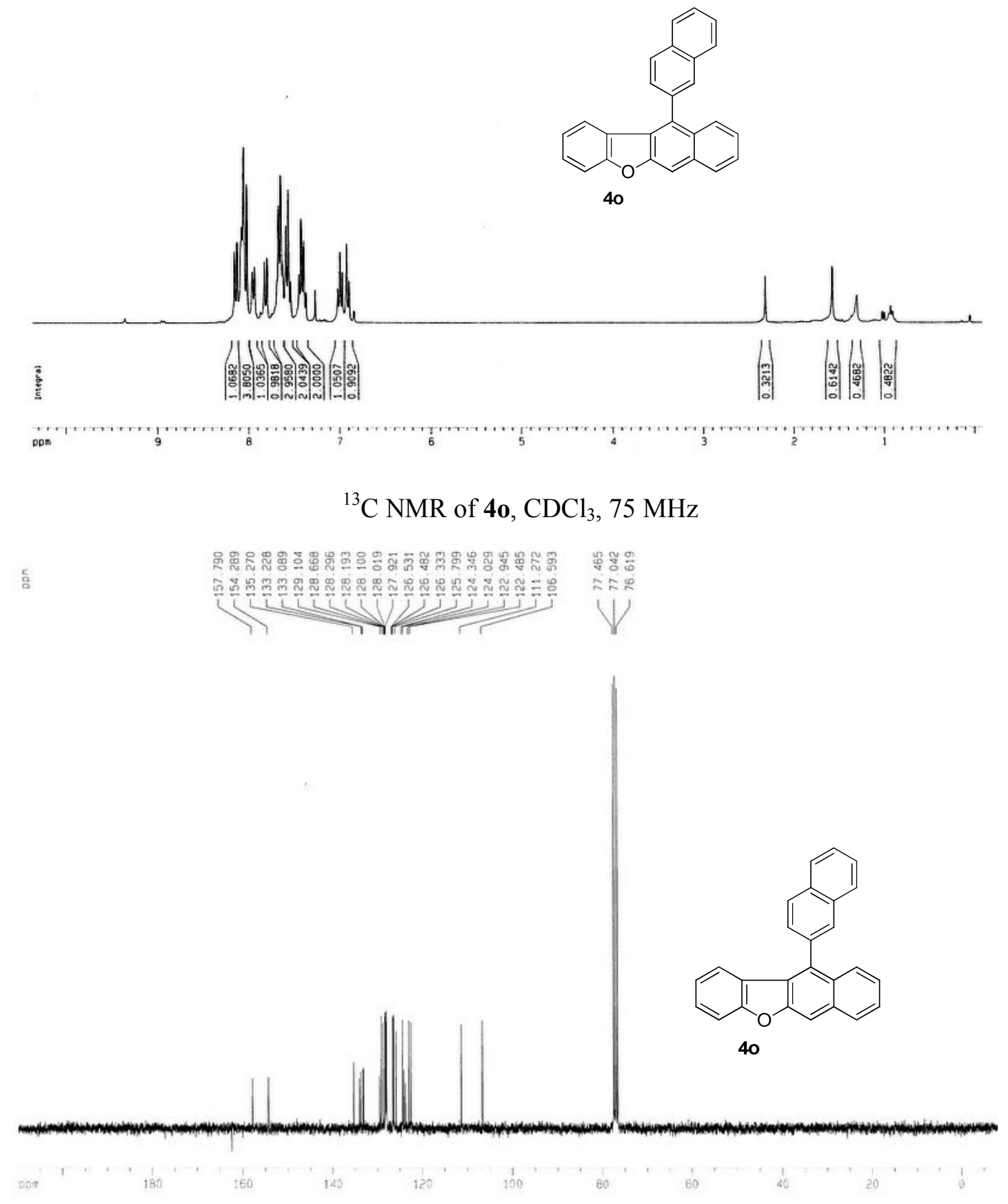
${ }^{1} \mathrm{H}$ NMR of $\mathbf{4 p}, \mathrm{CDCl}_{3} 300 \mathrm{MHz}$

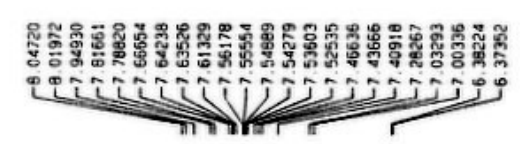

î

|
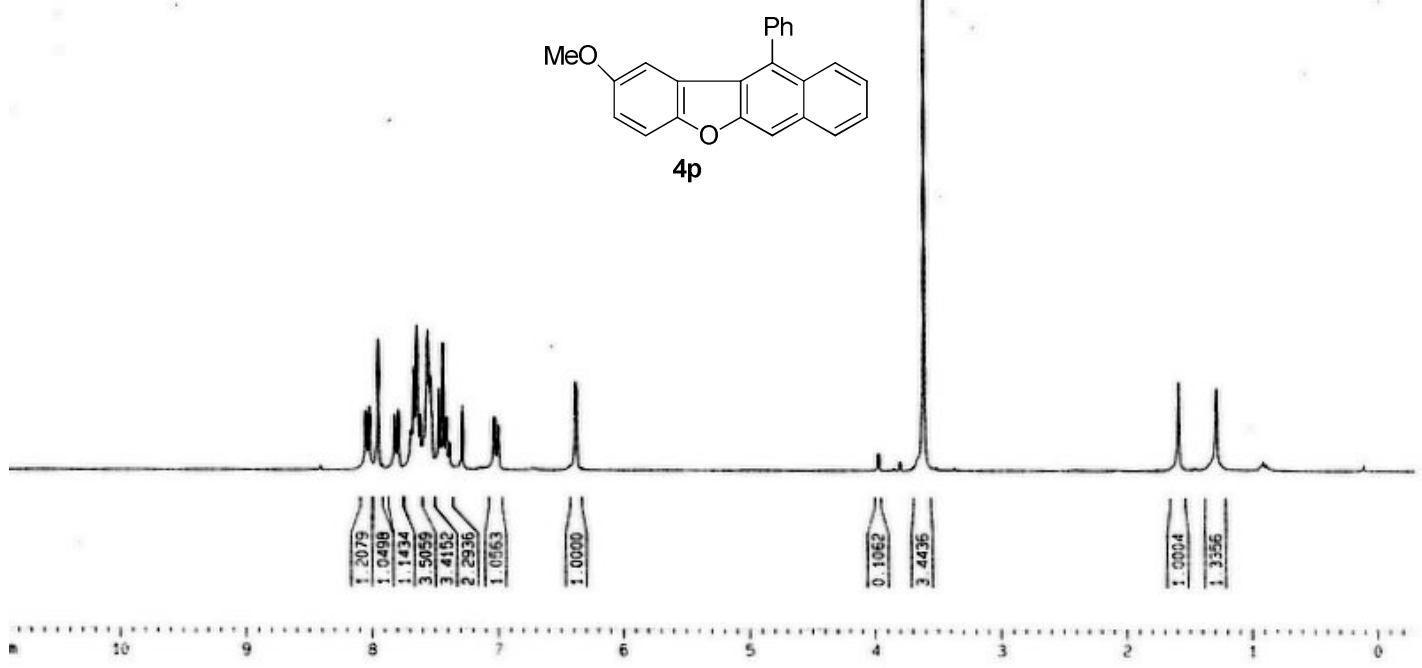

${ }^{13} \mathrm{C} \mathrm{NMR}$ of $4 \mathbf{p}, \mathrm{CDCl}_{3}, 75 \mathrm{MHz}$

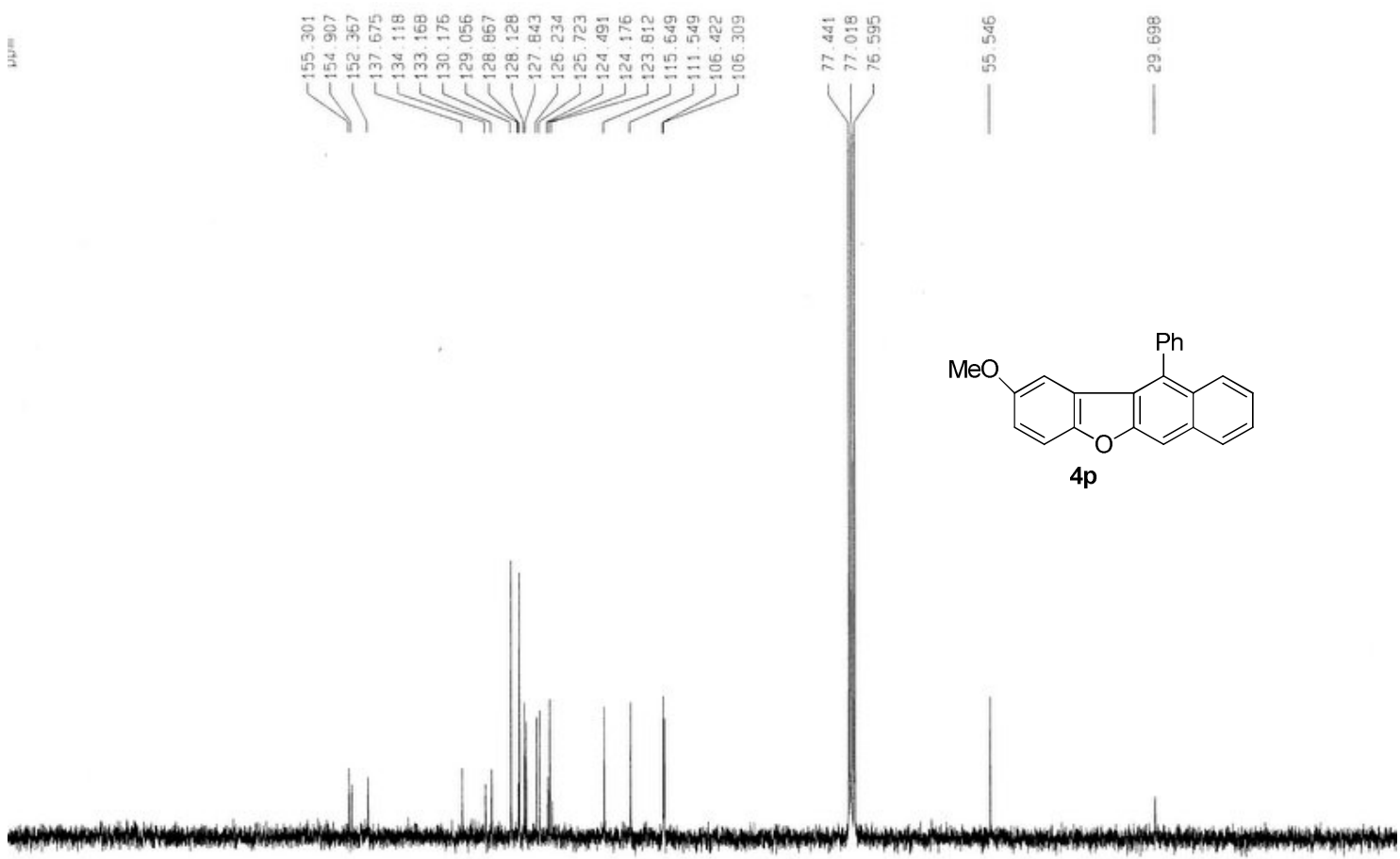


${ }^{1} \mathrm{H}$ NMR of $\mathbf{4 q}, \mathrm{CDCl}_{3} 300 \mathrm{MHz}$

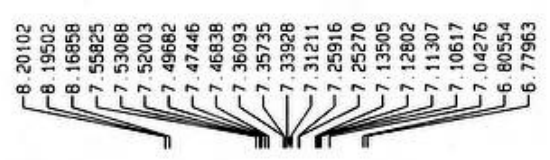

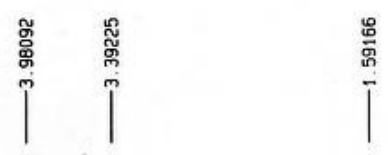
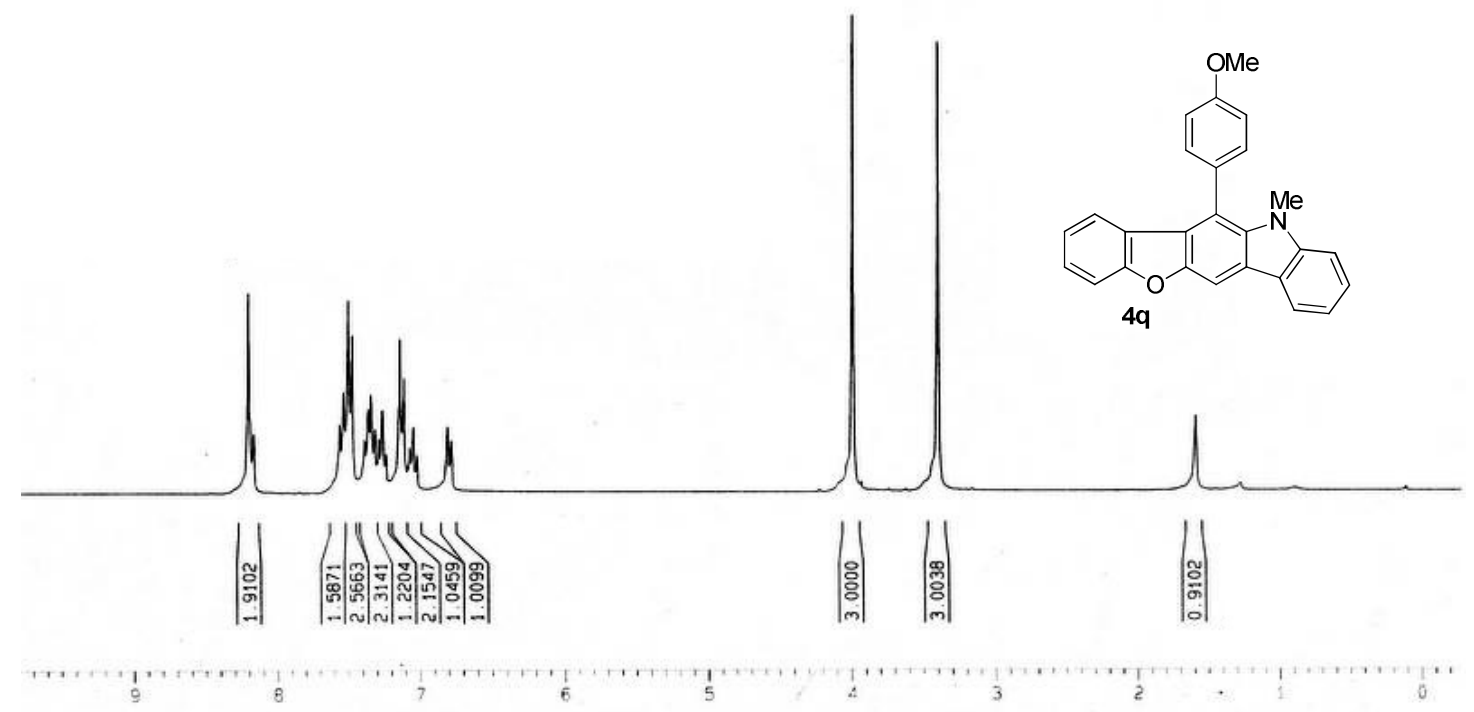

${ }^{13} \mathrm{C}$ NMR of $4 \mathbf{q}, \mathrm{CDCl}_{3}, 75 \mathrm{MHz}$
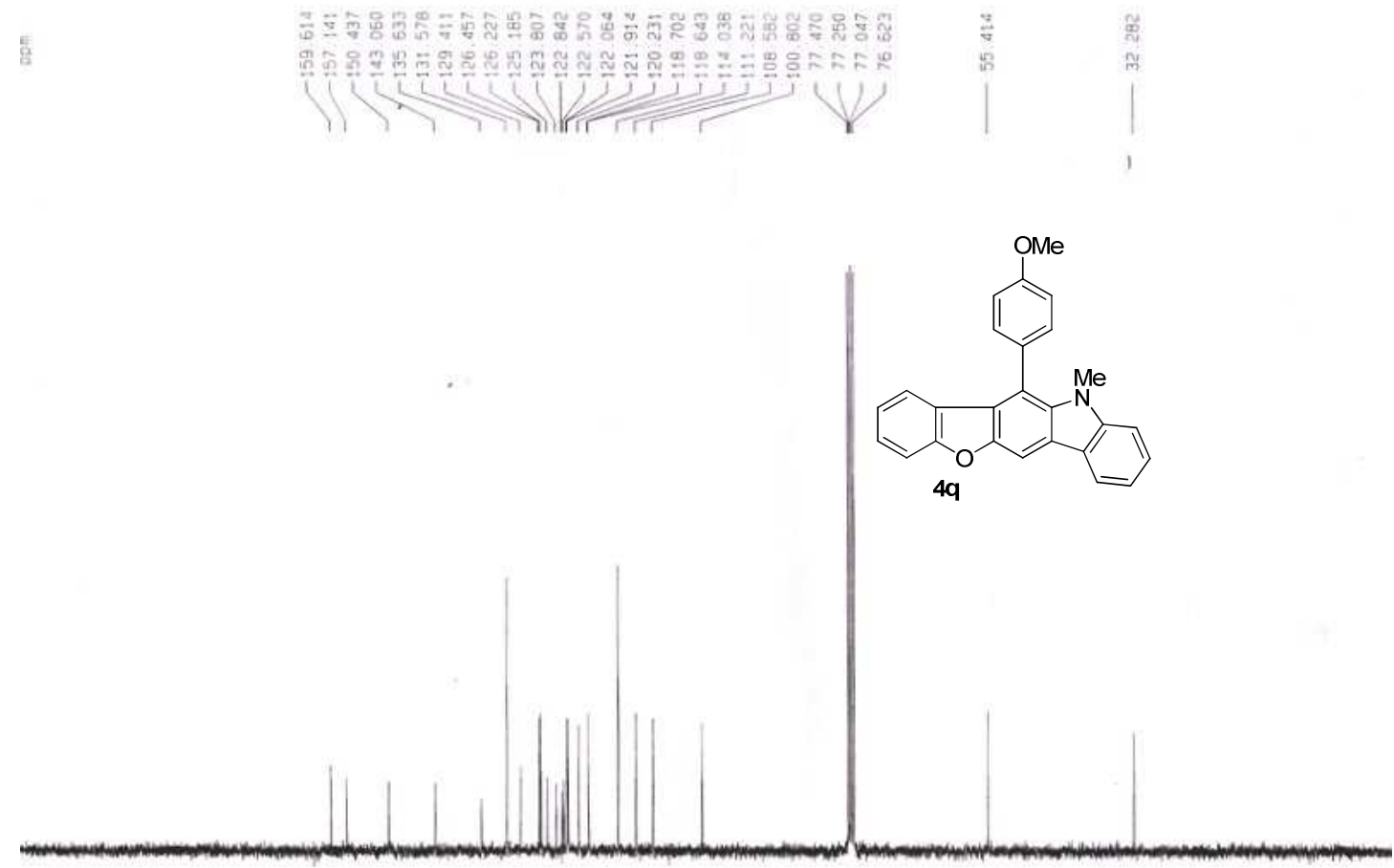
${ }^{1} \mathrm{H}$ NMR of $\mathbf{5 b}, \mathrm{CDCl}_{3}, 300 \mathrm{MHz}$

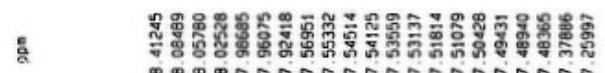

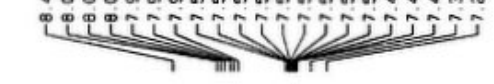

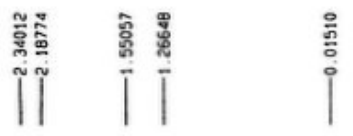

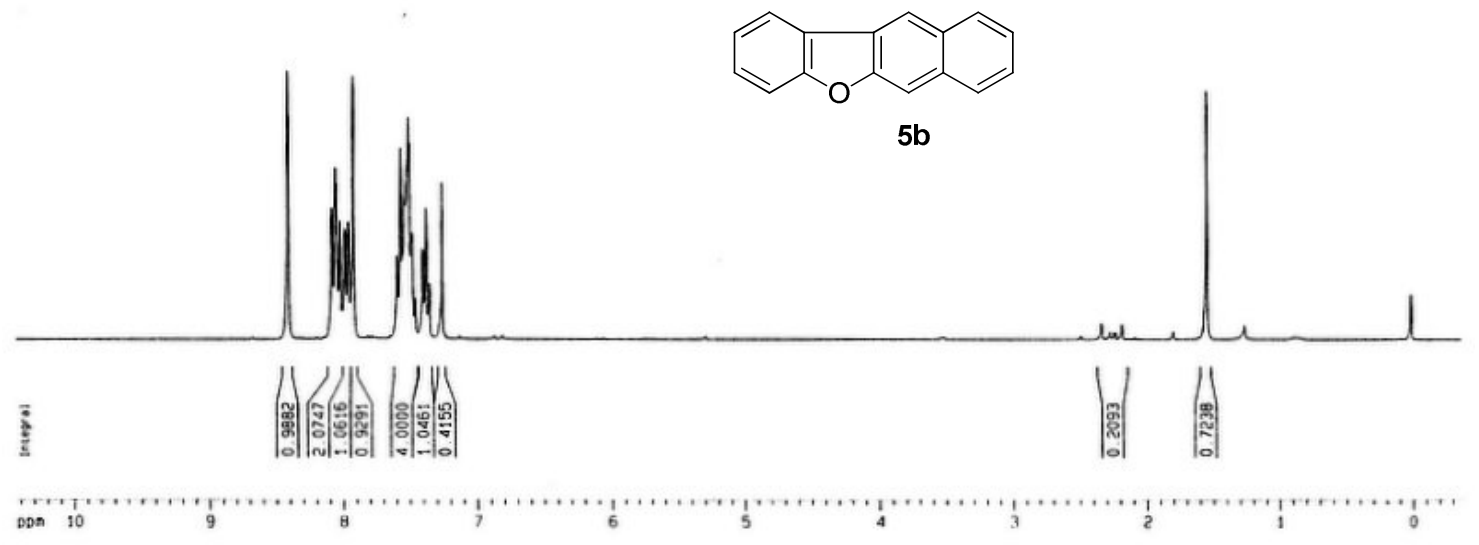

${ }^{1} \mathrm{H}$ NMR of $\mathbf{6 a}, \mathrm{CDCl}_{3}, 300 \mathrm{MHz}$

ฐ

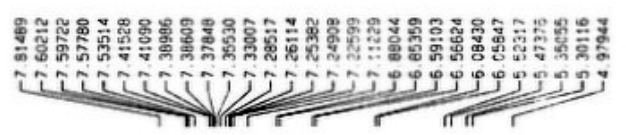

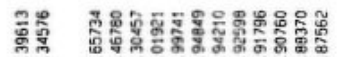

Vं<smiles>CC(=O)c1ccccc1C(=C1COc2ccccc21)c1ccccc1</smiles>

$6 a$

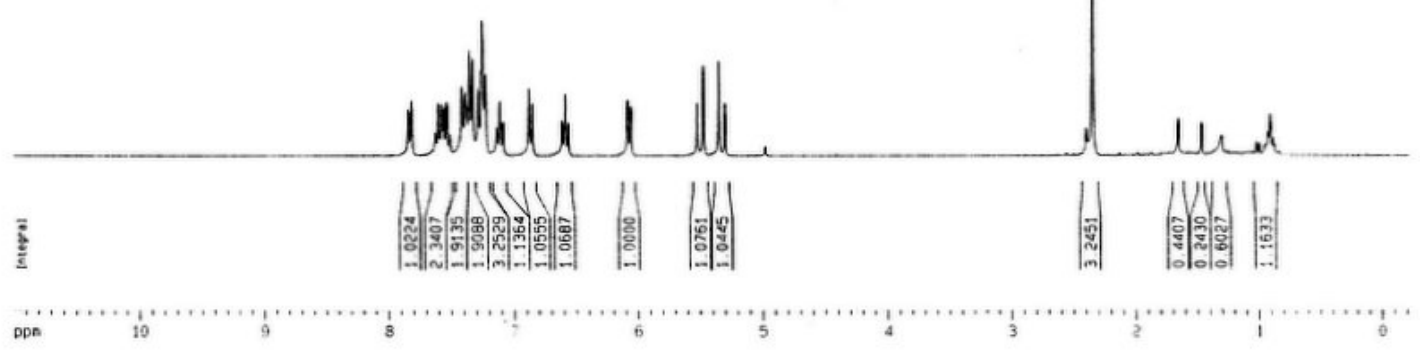




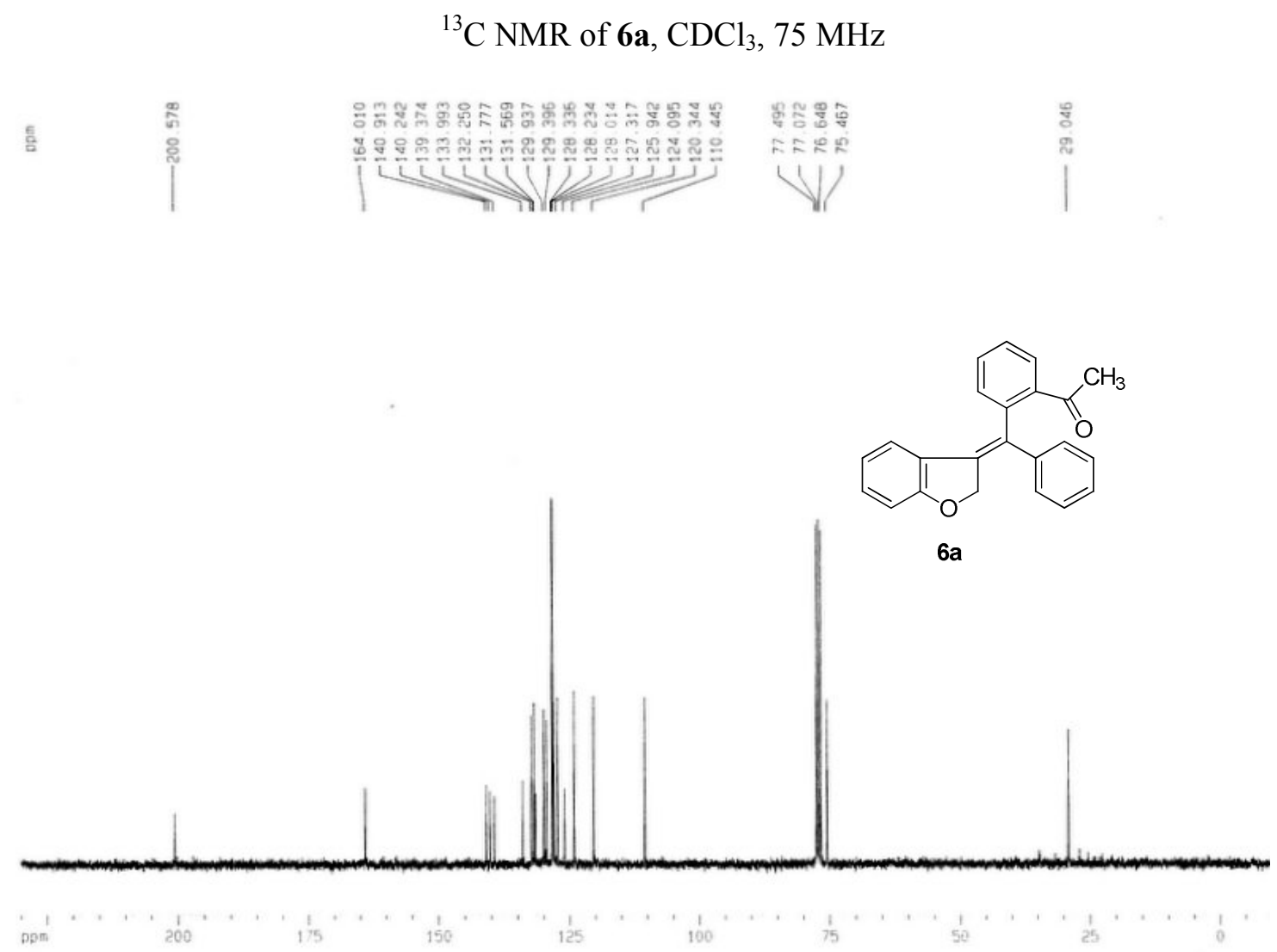

${ }^{1} \mathrm{H}$ NMR of $\mathbf{6 b}, \mathrm{CDCl}_{3}, 300 \mathrm{MHz}$
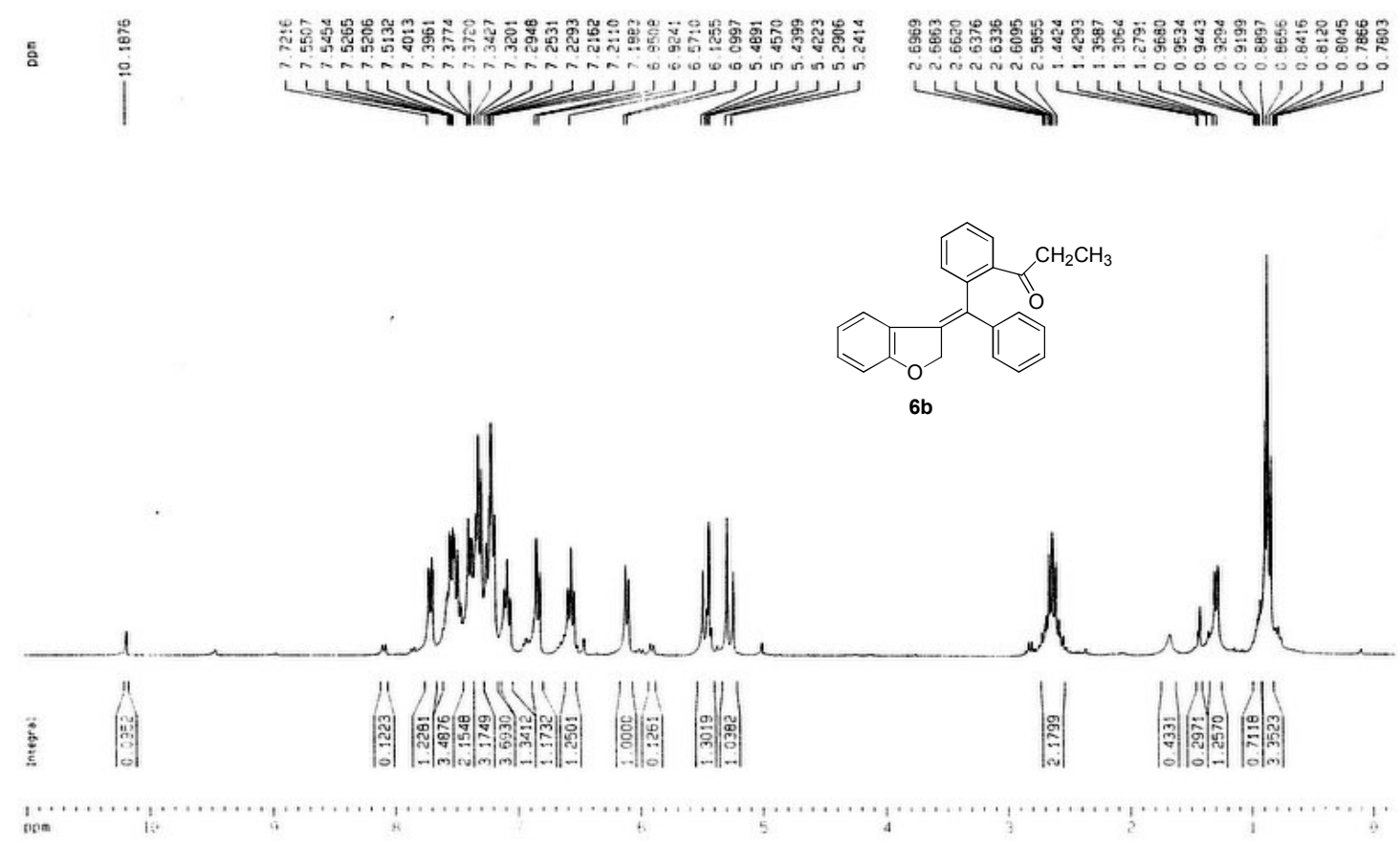


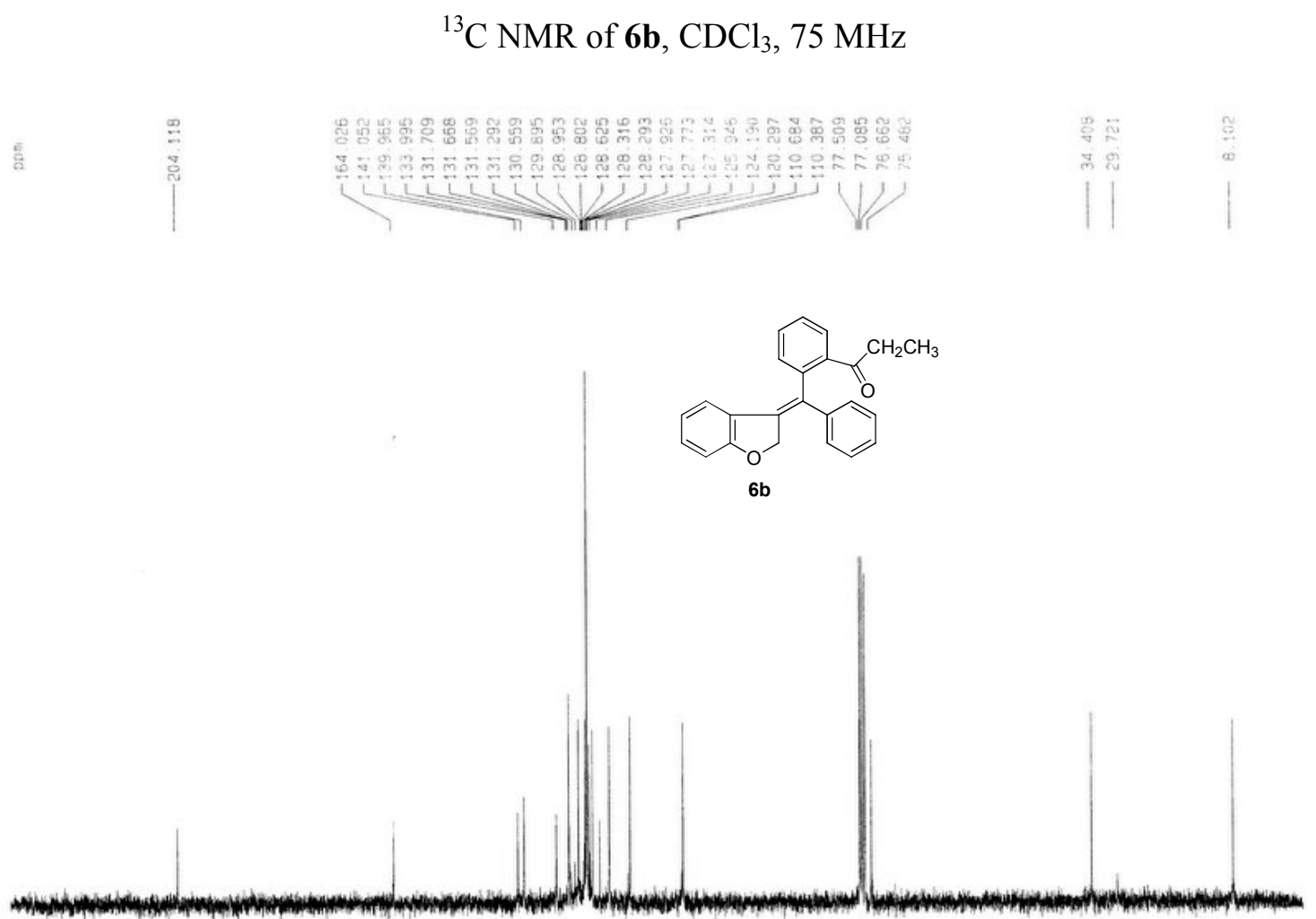

${ }^{1} \mathrm{H} \mathrm{NMR}$ of $\mathbf{7 a}, \mathrm{CDCl}_{3}, 300 \mathrm{MHz}$

ฐ

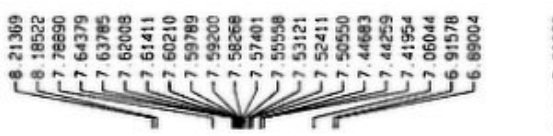

†
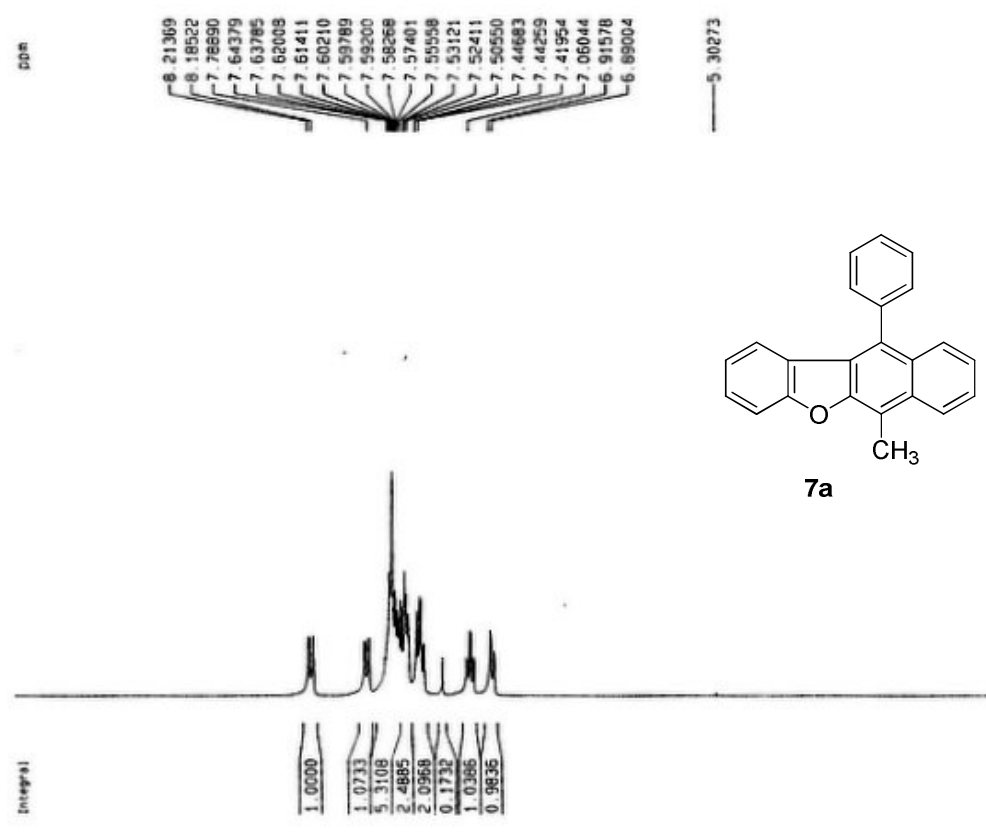

$7 a$

Dpm 

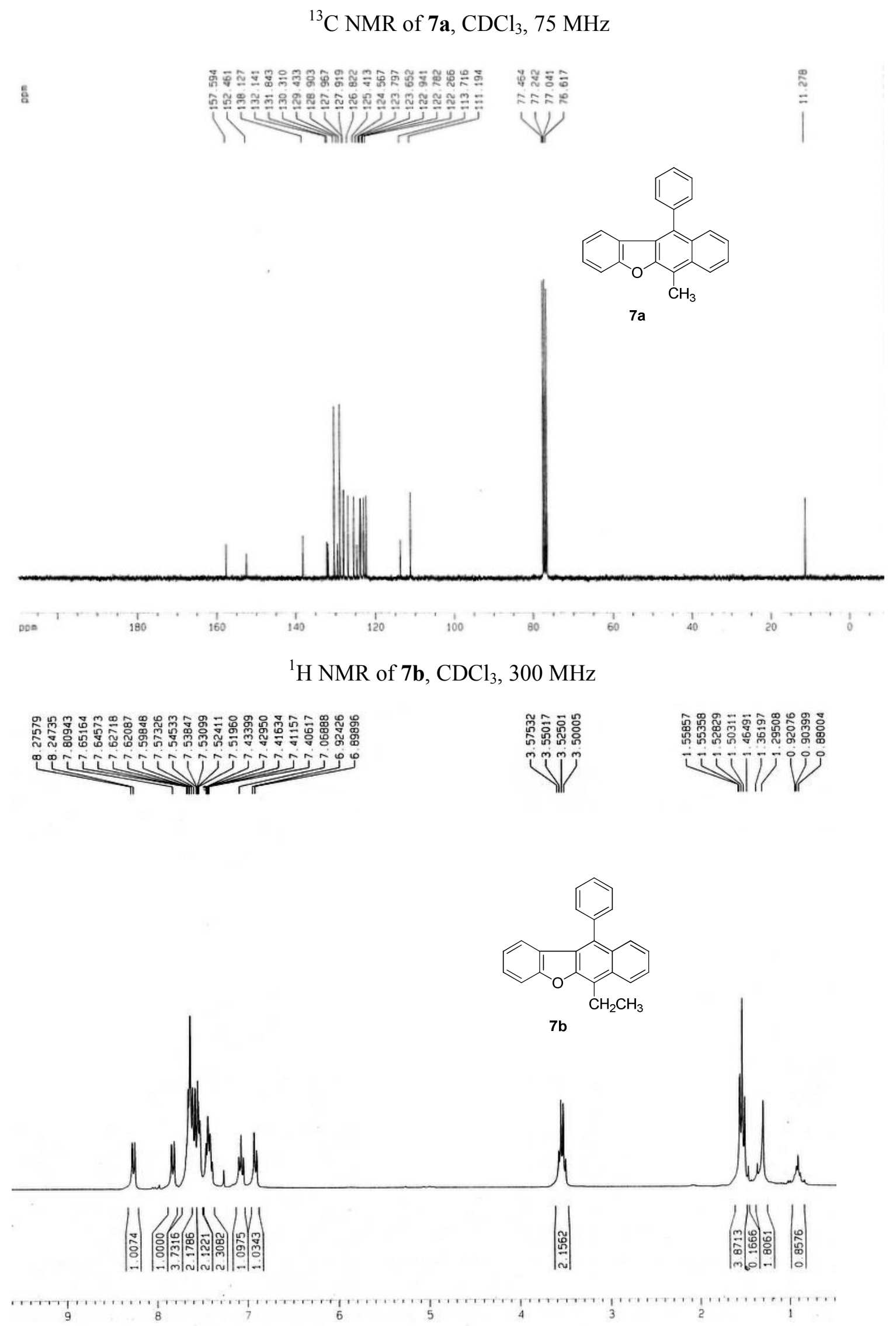


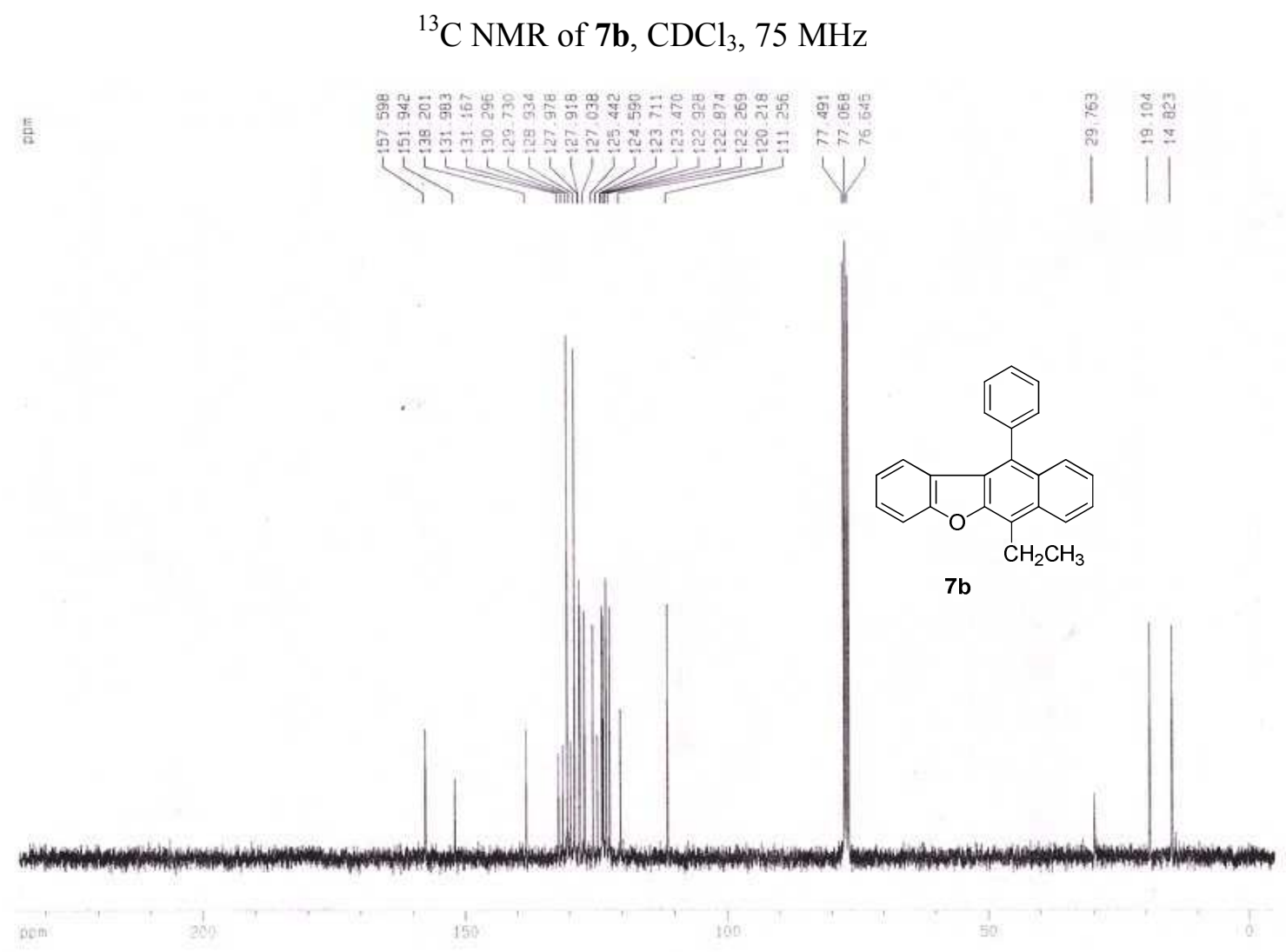


Crystallographic Data Collection and Refinement. The single crystal of compound 4a was mounted on the tips of glass fibers with commercially available glue. X-ray data collection of 4a was performed at room temperature using diffractometer equipped with a normal focus, sealed tube X-ray source with graphite monochromated Mo-K $\alpha$ radiation $(\lambda=0.71073 \AA)$. The data were integrated using $\mathrm{SAINT}^{1}$ program and the absorption corrections were made with SADABS. ${ }^{2}$ The structureof $\mathbf{4 a}$ was solved by SHELXS- $97^{3}$ usingPatterson method and followed by successive Fourier and difference Fourier synthesis. Full matrix least-squares refinements were performed on $\mathrm{F}^{2}$ using SHELXL-97 ${ }^{3}$ with anisotropic displacement parameters for all non-hydrogen atoms.All the hydrogen atoms were fixed geometrically by HFIX command and placed in ideal positions. Calculations were carried out using SHELXS $97,{ }^{3}$ SHELXL $97,{ }^{3}$ PLATON v1.15, ${ }^{4}$ ORTEP-3v2, ${ }^{5}$ and WinGX system Ver-1.80. ${ }^{6}$ Data collection and structure refinement parameters along with crystallographic data of $\mathbf{4 a}$ are given in Table 1.

\section{References}

1. SMART (V 5.628), SAINT (V 6.45a), XPREP, SHELXTL, Bruker AXS Inc., Madison, WI, 2004.

2. G. M. Sheldrick, SADABS (Version 2.03), University of Göttingen, Germany, 2002.

3. G. M. Sheldrick, SHELXS-97, Acta.Crystallogr.,2008, A64, 112.

4. A. L. Spek, Acta.Crystallogr.2009, D65, 148.

5. L. J. Farrugia, J. Appl. Crystallogr.1997, 30, 565.

6. L. J. Farrugia, WinGX,J. Appl. Crystallogr.1999, 32, 837.

Table 1.Crystallographic and Structural Refinement Parameters of 4a.

\begin{tabular}{|l|l|}
\hline Formula & $\mathrm{C}_{22} \mathrm{H}_{14} \mathrm{O}$ \\
\hline Formula Weight & 294.33 \\
\hline Crystal System & Triclinic \\
\hline Space group & $P \overline{1}$ \\
\hline$a / \AA$ & $9.1784(2)$ \\
\hline$b / \AA$ & $10.2343(2)$ \\
\hline$c / \AA$ & $10.5109(3)$ \\
\hline$\alpha /{ }^{\circ}$ & $98.755(2)$ \\
\hline$\beta /{ }^{\circ}$ & $113.273(2)$ \\
\hline$\gamma /{ }^{\circ}$ & $114.440(1)$ \\
\hline$V / \AA 3$ & $764.64(4)$ \\
\hline$Z$ & 2 \\
\hline$D_{\mathrm{c} / \mathrm{g} \mathrm{cm}^{-3}}$ & 1.278 \\
\hline$\mu / \mathrm{mm}^{-1}$ & 0.077 \\
\hline$F(000)$ & 308 \\
\hline$\theta$ range $^{\circ}$ & $2.3-27.5$ \\
\hline Reflections collected & 12329 \\
\hline
\end{tabular}




\begin{tabular}{|l|l|}
\hline Unique reflections & 3462 \\
\hline Reflections $I>2 \sigma(\mathrm{I})$ & 2110 \\
\hline$R_{\text {int }}$ & 0.026 \\
\hline goodness-of-fit $\left(F^{2}\right)$ & 1.03 \\
\hline$R 1(I>2 \sigma(\mathrm{I}))^{[\mathrm{a}]}$ & 0.0509 \\
\hline$w R 2(I>2 \sigma(\mathrm{I}))^{[\mathrm{a}]}$ & 0.1476 \\
\hline
\end{tabular}

$$
{ }^{[\mathrm{a}]} \mathrm{R}_{1}=\Sigma|| F_{\mathrm{o}}|-| F_{\mathrm{c}}|| / \Sigma\left|F_{\mathrm{o}}\right|, \mathrm{wR}_{2}=\left[\Sigma\left(w\left(F_{\mathrm{o}}{ }^{2}-{F_{\mathrm{c}}}^{2}\right)^{2}\right) / \Sigma w\left(F_{\mathrm{o}}{ }^{2}\right)^{2}\right]^{1 / 2}
$$

X-ray crystal structure of 4a (CCDC 143499)

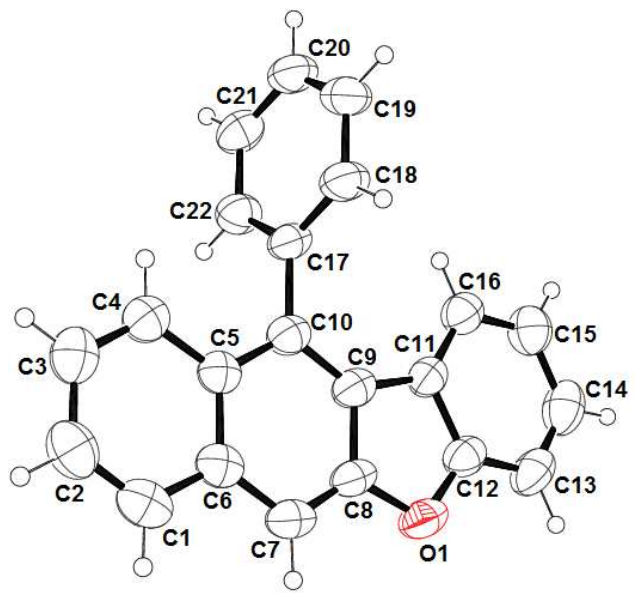

(50\% ellipsoidal probability) 